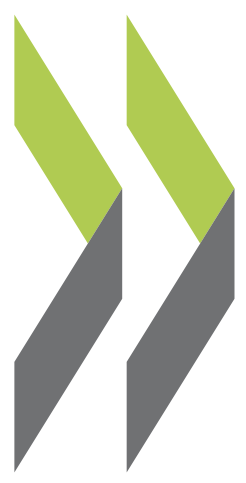

OECD Economics Department Working Papers No. 1560

Améliorer l'efficience de l'investissement public en

\title{
Pierre Guérin
}

France 
DEPARTEMENT DES AFFAIRES ECONOMIQUES

\section{AMÉLIORER L'EFFICIENCE DE L'INVESTISSEMENT PUBLIC EN FRANCE DÉPARTEMENT DES AFFAIRES ÉCONOMIQUES DOCUMENT DE TRAVAIL No. 1560}

\section{Par Pierre Guérin}

Les documents de l'OCDE ne doivent pas être présentés comme exprimant les vues officielles de l'OCDE ou de ses pays membres. Les opinions exprimées et les arguments sont ceux des auteur(s).

Accord pour la publication par Isabell Koske, Directeur adjoint, Branche Études par pays, Département des affaires économiques.

Les Documents de travail du Département des Affaires économiques sont disponibles à www.oecd.org/eco/workingpapers.

JT03449637 
Les documents de travail de l'OCDE ne doivent pas être présentés comme exprimant les vues officielles de l'OCDE ou de ses pays membres. Les opinions exprimées et les arguments employés sont ceux des auteurs.

Les documents de travail exposent des résultats préliminaires ou des travaux de recherche en cours menés par l'auteur/les auteurs et sont publiés pour stimuler le débat sur un large éventail de questions sur lesquelles l'OCDE travaille.

Les commentaires sur les documents de travail sont les bienvenus et peuvent être adressés au Département des Affaires économiques, OCDE, 2 rue André-Pascal, 75775 Paris Cedex 16, France ou à l'adresse mail suivante : eco.contact@oecd.org.

Les Documents de travail du Département des Affaires économiques sont disponibles à www.oecd.org/eco/workingpapers.

Le contenu ainsi que les données et toute carte qui y figurent sont sans préjudice du statut de tout territoire, de la souveraineté s'exerçant sur ce dernier, du tracé des frontières et limites internationales, et du nom de tout territoire, toute ville ou toute région.

Les données statistiques concernant Israël sont fournies par et sous la responsabilité des autorités israéliennes compétentes. L'utilisation de ces données par l'OCDE est sans préjudice du statut des hauteurs du Golan, de Jérusalem Est et des colonies de peuplement israéliennes en Cisjordanie aux termes du droit international.

\section{(c) OCDE (2019)}

Vous êtes autorisés à copier, télécharger ou imprimer du contenu OCDE pour votre utilisation personnelle. Vous pouvez inclure des extraits des publications, des bases de données et produits multimédia de l'OCDE dans vos documents, présentations, blogs, sites Internet et matériel d'enseignement, sous réserve de faire mention de la source OCDE et du copyright. Les demandes pour usage commercial ou de traduction devront être adressées à rights@oecd.org. 


\begin{abstract}
RÉSUMÉ
Améliorer l'efficience de l'investissement public en France

L'investissement public a le potentiel de stimuler la demande à court terme, et des infrastructures appropriées peuvent aussi entrainer des gains de productivité et contribuer à rendre la croissance économique plus inclusive en garantissant une égalité d'accès au marché du travail, à l'éducation et aux soins de santé. Les investissements publics de la France sont élevés, et son stock de capital public est important. En particulier, le réseau d'infrastructures de la France est bien développé, et très bien classé si on le compare à celui d'autres pays. Pour autant, dans certains secteurs, le manque d'investissements d'entretien et de modernisation a sans doute nui à l'efficacité du capital investi dans les infrastructures existantes. Pour pouvoir bénéficier pleinement de ses investissements publics, la France doit renforcer et développer l'ensemble de sa stratégie pour relever plusieurs défis, consistant notamment à promouvoir les considérations d'efficience socio-économique dans le choix de ses projets d'investissement, à harmoniser les procédures s'étalant sur toute la durée de vie des investissements entre les différents secteurs et niveaux administratifs, et à concentrer les investissements de certains secteurs sur l'entretien et la qualité du stock de capital. En outre, comme les collectivités locales sont responsables de la plupart des investissements publics, il sera utile de poursuivre les efforts visant à répartir clairement les responsabilités entre les différents échelons administratifs pour pouvoir bénéficier d'économies d'échelle et de gamme. L'investissement public devrait aussi viser à atteindre les objectifs fixés dans les domaines de l'environnement, du numérique, de la recherche et de l'innovation pour permettre à la France de respecter ses engagements nationaux et internationaux et garantir une croissance plus vigoureuse et plus durable. Les choix opérés devront faire une place à l'investissement privé pour permettre la réalisation de ces objectifs et stimuler la croissance potentielle, notamment en développant une liste de projets prêts à financer et en ajustant de manière dynamique le soutien public aux politiques en matière d'énergie et de changement climatique.
\end{abstract}

Ce Document de travail se rapporte à l'Étude économique de l'OCDE de la France 2019

(http://www.oecd.org/fr/economie/france-en-un-coup-d-oeil/)

Classification JEL : H54; H71; O38; Q48; R42

Mots clefs : Infrastructure; Transport; Energie; Innovation.

$* * * * * * * * * *$

\title{
Improving the efficiency of public investment in France
}

Public investment can stimulate demand in the short term and suitable infrastructure can also raise productivity and contribute to more inclusive economic growth by ensuring equal access to the labour market, education and health care. France makes substantial public investments and its stock of public capital is large. In particular, France's infrastructure network is well developed and ranks favourably in international comparison. Yet, the capital efficiency of existing infrastructure may have been hampered by a lack of maintenance and upgrading investments in some sectors. To take full advantage of its public investment, France must strengthen and develop its strategy to meet several challenges, including by promoting socioeconomic-efficiency considerations in the selection of investment projects, harmonising procedures extending over the life-cycle of investment projects between sectors and administrative levels, and concentrating investments of some sectors on the maintenance and quality of the capital stock. Moreover, as local governments are responsible for most of public investment, continuing efforts to clearly allocate responsibilities across the different levels of government will be helpful to reap the benefits of economies of scale and scope. Public investment should also be geared towards fulfilling environmental, digital, and research and innovation objectives to meet France's domestic and international commitments and secure stronger and more sustainable growth. Public investment choices will need to crowd-in private investment to meet these objectives and raise potential growth, including by developing a pipeline of ready-to-finance projects and dynamically adjusting public support to energy and climate change policies.

This Working Paper relates to the 2019 OECD Economic Survey of France (http://www.oecd.org/economy/surveys/france-economic-snapshot/)

JEL codes: H54; H71; O38; Q48; R42

Keywords: Infrastructure; Transport; Energy; Innovation. 


\section{Table des matières}

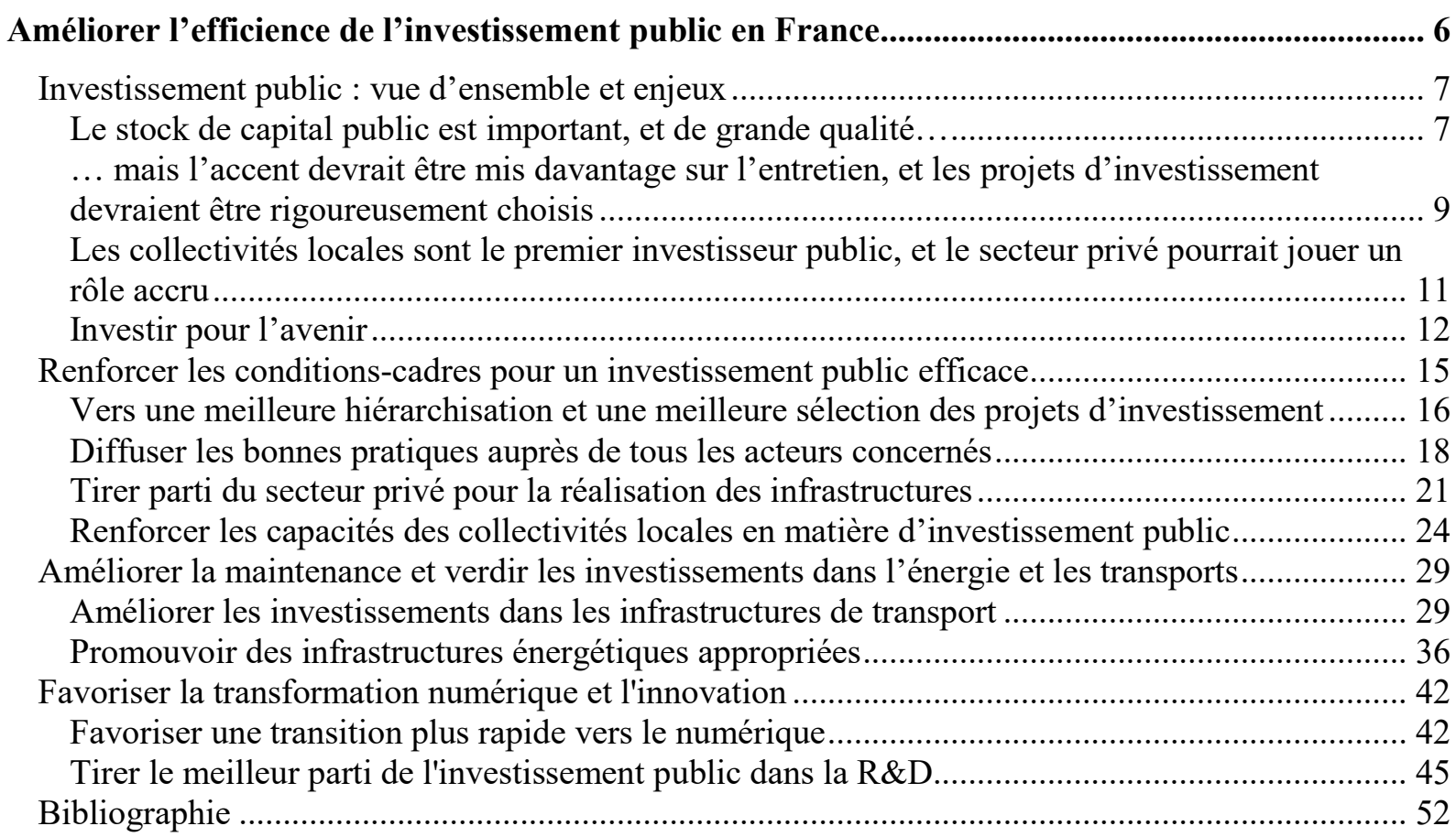

\section{Tableaux}

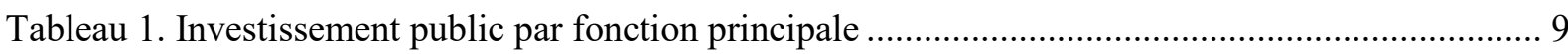

Tableau 2. Principes de l'OCDE pour un investissement public efficace ........................................... 16

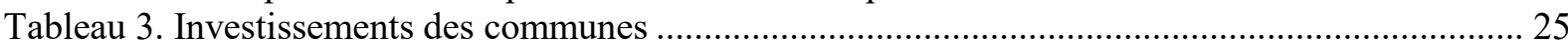

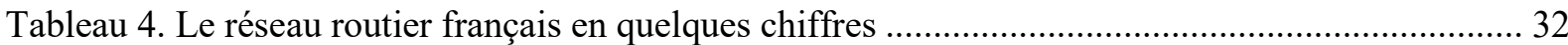

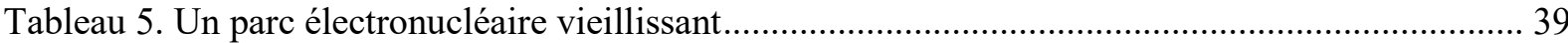

\section{Graphiques}

Graphique 1. Stock de capital public et investissement public

Graphique 2. Indicateurs de la gouvernance des infrastructures ...................................................... 10

Graphique 3. Répartition des dépenses d'investissement entre les différents niveaux de

l'administration

Graphique 4. Investissements des collectivités locales

Graphique 5. Le financement public de la recherche est proche de la moyenne OCDE ....................... 15

Graphique 6. Le stock de capital financé par le biais de PPP est plutôt limité .................................... 22

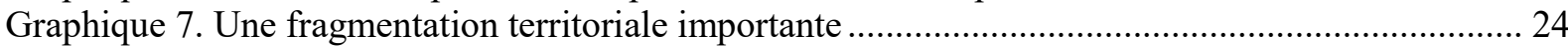

Graphique 8. Dépenses d'investissement des communes par type de municipalités ............................ 25 
Graphique 9. Dépenses d'investissement des collectivités locales en pourcentage du total des dépenses 27

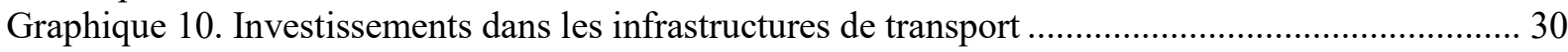

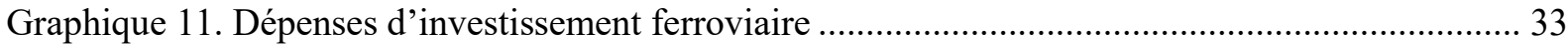

Graphique 12. Le fret ferroviaire est engagé sur la mauvaise voie ................................................... 35

Graphique 13. Peu d'énergies renouvelables dans le parc électrique, mais peu d'émissions de CO2

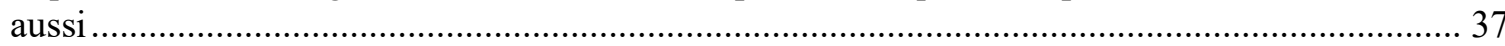

Graphique 14. Des gains d'efficacité énergétique qui proviennent essentiellement du secteur

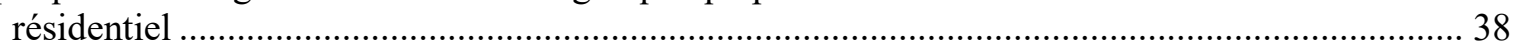

Graphique 15. Une production d'électricité d'origine principalement nucléaire .................................. 39

Graphique 16. Une faible capacité d'interconnexion avec le marché européen de l'électricité............ 42

Graphique 17. Il est possible d'améliorer la qualité des infrastructures à haut débit............................ 43

Graphique 18. La pénétration des technologies numériques dans l'administration publique

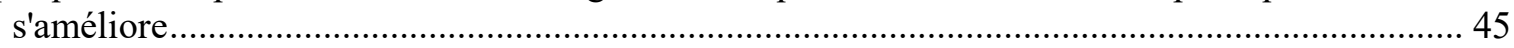

Graphique 19. Les dépenses de recherche-développement $(R \& D)$ correspondent peu ou prou à la

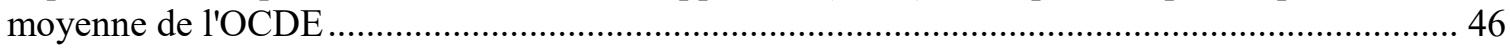

Graphique 20. Flux internationaux nets d'auteurs scientifiques ........................................................ 48

Graphique 21. Le financement sur projet des activités de recherche-développement (R\&D) est limité.

Graphique 22. L'intensité de recherche-développement (R\&D) des entreprises est plus forte une fois corrigée de la structure d'activités

\section{Encadrés}

Encadré 1. Définir et mesurer l'investissement public : exemples de difficultés rencontrées ................ 7 Encadré 2. Le Grand plan d'investissement et le Fonds pour l'innovation et l'industrie....................... 13 Encadré 3. Processus décisionnel des grands projets d'investissement hospitalier............................... 19 Encadré 4. Répartition des responsabilités et planification des transports ............................................ 31 


\title{
Améliorer l'efficience de l'investissement public en France
}

\author{
Par Pierre Guérin ${ }^{1}$
}

L'investissement public peut dynamiser l'investissement privé et contribuer à rendre la croissance plus inclusive. En ce sens, développer l'investissement public peut venir étayer la croissance et accroître le bien-être. Cela étant, les capacités d'investissement sont limitées par les contraintes qui pèsent sur les ressources publiques. De ce fait, il est nécessaire de maximiser le rendement de l'investissement public par une sélection rigoureuse des projets. Les politiques propres à améliorer l'efficience de l'investissement public seront passées en revue dans le présent chapitre.

Définir ce qu'est l'investissement public n'est toutefois pas une tâche facile (Encadré 1). D'un point de vue comptable, l'investissement public désigne des dépenses en capital consacrées à des infrastructures physiques ou immatérielles dont la durée d'utilisation productive est supérieure à un an (la formation brute de capital fixe ou FBCF). Cependant, les questions examinées dans le présent chapitre vont au-delà de cette définition stricte, soit parce que les opérations concernées pourraient être classées comme consommation intermédiaire (opérations d'entretien et de maintenance par exemple), soit parce que l'entité à l'origine des investissements n'est pas considérée comme faisant partie du secteur public d'un point de vue statistique. De plus, les investissements en infrastructures évoqués dans ce chapitre ne comprennent pas les biens privés fournis par la puissance publique comme l'éducation, la santé ou le logement social. Au contraire, suivant Cournède et Ziemann (2019), les politiques sectorielles présentées dans les parties 3 et 4 mettent l'accent sur les investissements en infrastructures qui bénéficient à des secteurs autres que celui qui possède les actifs, à savoir le transport, l'énergie, le numérique et la recherche.

1. Pierre Guérin est économiste au Département des Affaires économiques de l'OCDE (pierre.guerin@oecd.org). L'auteur souhaite remercier Isabell Koske, Dorothée Allain-Dupré, Pierre Beynet, Isabelle Chatry, Andrés Fuentes Hutfilter, Antoine Goujard, Jan-Horst Keppler, Patrick Lenain, Dejan Makovsek, Sarah Perret, Frédéric Sgard, Kurt Van Dender, Frédérique Zegel et Volker Ziemman pour leurs précieux commentaires. Le chapitre a également bénéficié des commentaires des autorités françaises. Un remerciement particulier pour Patrizio Sicari pour la recherche statistique et à Sylvie Ricordeau pour son aide éditoriale. 


\section{Encadré 1. Définir et mesurer l’investissement public : exemples de difficultés rencontrées}

En fonction de leur ampleur, les opérations d'entretien peuvent être classées soit dans la consommation intermédiaire, soit dans la FBCF. Par exemple, environ $25 \%$ des dépenses de voirie effectuées par les collectivités locales sont considérées comme des consommations intermédiaires et non comme des investissements (Gouvernement, 2017a). En outre, il existe en France un grand nombre d'entreprises publiques, qui représentaient quelque $7 \%$ de l'emploi total en 2015 (OCDE, 2017a). Or, les investissements réalisés par les entreprises publiques qui ne sont pas classées dans les administrations publiques à des fins statistiques parce que leurs recettes commerciales sont supérieures à $50 \%$ de leurs coûts de production représentent environ $1.1 \%$ du PIB chaque année (Gouvernement, 2017b). En fonction de la nature du contrat, les partenariats public-privé ne sont pas non plus forcément inclus dans la FBCF. Par exemple, les investissements réalisés dans le cadre de marchés de partenariat où le secteur privé est chargé de concevoir, construire, financer et entretenir une structure ou une installation pour une mission de service public, sont généralement inclus dans la FBCF publique, tandis que d'autres contrats comme les concessions de service et non de travaux ne le sont généralement pas.

On trouvera dans la première partie de ce chapitre une vue d'ensemble de l'investissement public en France et une présentation des enjeux qui lui sont associés. La section suivante est consacrée aux conditions cadres permettant d'améliorer l'efficacité de l'investissement public, en insistant sur la plus grande place qu'il conviendrait de faire aux considérations d'efficience économique pour aider à hiérarchiser les projets d'investissement, au rôle du secteur privé dans la fourniture d'infrastructures, et aux réformes institutionnelles et budgétaires qui pourraient aider à tirer le meilleur parti des investissements locaux et favoriser la croissance. La troisième partie traite principalement de deux secteurs dans lesquels le stock de capital est important en France, le transport et l'énergie, et dans lesquels les priorités d'investissement devraient être ciblées sur l'entretien et le respect des objectifs environnementaux. Enfin, les questions relatives au soutien public propre à promouvoir la transition vers une économie numérique et à stimuler la recherche et l'innovation sont examinées dans la dernière partie.

\section{Investissement public : vue d'ensemble et enjeux}

\section{Le stock de capital public est important, et de grande qualité...}

Le stock de capital public de la France est important et si l'on se réfère aux enquêtes disponibles, la qualité de ses infrastructures est élevée (Graphique 1, parties A et B). Si l'investissement public a reculé ces dernières années et se situe désormais dans la moyenne de l'OCDE, il a été largement plus élevé que dans d'autres pays de la zone euro (parties C et D). L'investissement public par principale fonction économique est assez semblable au profil moyen de l'investissement public des pays de l'OCDE (Tableau 1). Si l'on compare les résultats de la France à une moyenne de pays de la zone euro, on voit que l'investissement public y est considérablement plus vigoureux, ce qui reflète en partie la baisse de l'investissement public qui, à partir de 2011, a touché les pays qui avaient été le plus affectés par la crise de la zone euro, mais aussi le niveau plus important des investissements dans la défense (Graphique 1, Panel D). 
Graphique 1. Stock de capital public et investissement public

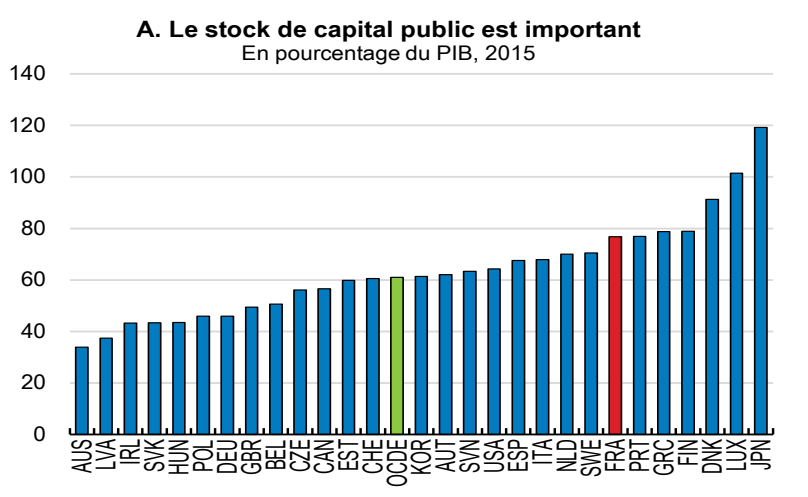

C. L'investissement public est proche de la moyenne de l'OCDE En pourcentage du PIB, 2018 ou dernière année disponible

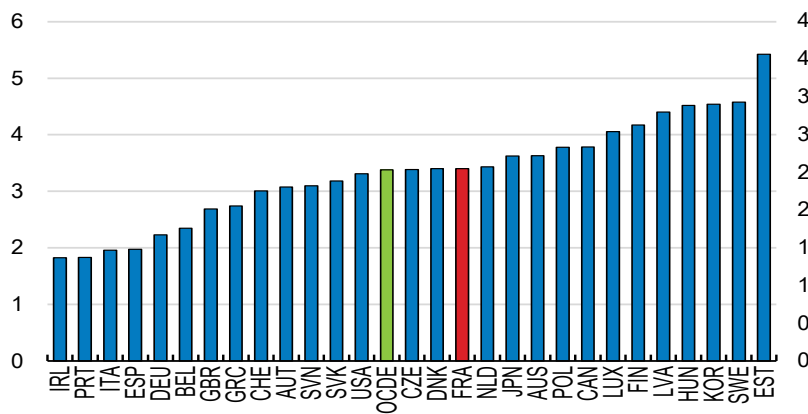

B. La qualité des infrastructures est élevée Indice ${ }^{1}, 2017$

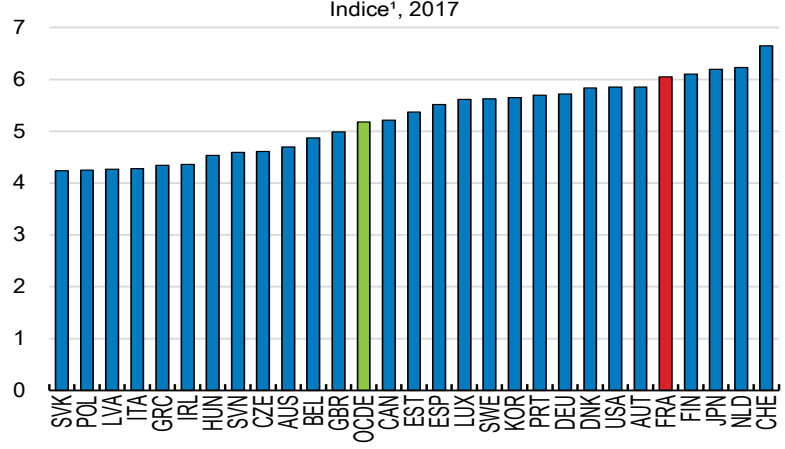

D. L'investissement public a diminué ces dernières années

En pourcentage du PIB

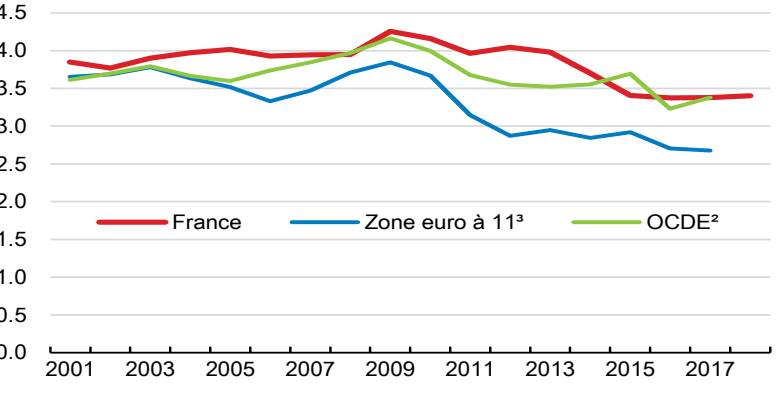

1. Indice variant de 0 (qualité subjective minimale) à 7 (qualité subjective maximale), calculé à partir des appréciations fournies par des responsables d'entreprises opérant dans le pays en réponse à la question : " Comment évaluez-vous l'état général des infrastructures (par exemple transport, communication et énergie) dans votre pays?".

2. Hors Chili et Mexique en raison de disponibilité limitée des données ; moyenne non pondérée.

3. ZE-11 (zone euro-11) correspond à la moyenne non pondérée des pays suivants : Allemagne, Autriche, Belgique, Espagne, Finlande, Grèce, Irlande, Italie, Luxembourg, Pays-Bas et Portugal.

Source : FMI (2017), Investment and Capital Stock Statistics (base de données), Fonds monétaire international, Washington D.C.; WEF (2018), The Global Competitiveness Report 2017-2018 (base de données), Forum économique mondial, Genève ; et OCDE (2019), Perspectives économiques de l'OCDE : statistiques et projections (base de données) et mises à jour.

StatLink त्गा5म https://doi.org/10.1787/888933952651 
Tableau 1. Investissement public par fonction principale

Investissement public par fonction principale

\begin{tabular}{lccccc}
\hline & France & OCDE $^{2}$ & $\begin{array}{c}\text { France - OCDE } \\
\text { Écart }\end{array}$ & ZE-1133 & $\begin{array}{c}\text { France-ZE-11 } \\
\text { Écart }\end{array}$ \\
\hline \multicolumn{1}{c}{ Total } & 3.9 & 3.7 & 0.2 & 3.3 & 0.6 \\
\hline Services publics généraux & 0.5 & 0.5 & -0.0 & 0.5 & -0.0 \\
Défense & 0.3 & 0.3 & 0.0 & 0.2 & 0.1 \\
Ordre public et sécurité & 0.1 & 0.1 & 0.0 & 0.1 & 0.0 \\
Affaires économiques & 1.3 & 1.3 & -0.1 & 1.2 & 0.0 \\
Protection de l'environnement & 0.2 & 0.2 & 0.0 & 0.2 & 0.0 \\
Logement et équipements collectifs & 0.3 & 0.2 & 0.1 & 0.2 & 0.1 \\
Santé & 0.4 & 0.3 & 0.1 & 0.2 & 0.1 \\
\hline Loisirs, culture et religion & 0.3 & 0.2 & 0.1 & 0.2 & 0.1 \\
Éducation & 0.4 & 0.5 & -0.1 & 0.4 & 0.0 \\
Protection sociale & 0.1 & 0.1 & 0.0 & 0.1 & 0.0 \\
\hline
\end{tabular}

1. Les chiffres peuvent ne pas correspondre aux totaux du fait que les chiffres sont arrondis.

2. OCDE est la moyenne non pondérée de 27 pays de l'OCDE pour lesquels des données sont disponibles (hors France). Par conséquent, cela ne correspond pas exactement au groupe de pays de l'OCDE présenté dans le graphique 1, qui comprend tous les pays de l'OCDE.

3. ZE-11 est la moyenne non pondérée des pays suivants: Allemagne, Autriche, Belgique, Espagne, Finlande, Grèce, Irlande, Italie, Luxembourg, Pays-Bas et Portugal.

Source : OCDE (2018), Statistiques de l'OCDE sur les comptes nationaux (base de données).

\section{... mais l'accent devrait être mis davantage sur l'entretien, et les projets d'investissement devraient être rigoureusement choisis}

$\mathrm{Si}$, en termes de qualité et de gouvernance des infrastructures, la France est bien classée au niveau international (Graphique 2), l'investissement dans l'entretien n'a sans doute pas toujours été approprié dans certains secteurs comme le secteur ferroviaire, où la priorité a été donnée à de nouveaux projets au détriment de la maintenance du réseau existant (Cour des comptes, 2014). De même, un tiers du parc immobilier universitaire est jugé dégradé. Dans le passé, des plans d'investissement ponctuels, concernant par exemple la rénovation d'hôpitaux ou d'universités, ont aussi été adoptés pour pallier le manque d'investissements d'entretien et de modernisation des infrastructures. Pourtant, les besoins globaux d'investissement pourraient être réduits et l'efficience du capital accrue si l'on assurait un niveau élevé de maintenance des infrastructures existantes. Il est aussi possible que les avantages liés à de nouveaux investissements publics diminuent lorsque le stock de capital public est déjà élevé, et les risques d'éviction de l'investissement privé peuvent aussi être plus importants.

Une planification stratégique rigoureuse des investissements pour hiérarchiser les projets est une condition nécessaire à des investissements de qualité, et la France se caractérise par une longue tradition de planification des investissements dans les secteurs de l'énergie et des transports. Afin de développer une telle hiérarchisation, tous les grands projets d'investissement public (hors défense et investissements intégralement financés par les collectivités locales) doivent obligatoirement faire l'objet d'une évaluation socioéconomique ex ante depuis 2013. Cette pratique est très répandue dans le secteur des transports, car elle y est obligatoire depuis 1982, et dans le secteur de la santé, des procédures spécifiques sont mises en œuvre depuis 2013 pour garantir la qualité de l'évaluation des projets d'investissement. Par ailleurs, bien que la pratique de l'évaluation socio-économique des projets d'investissement commence à s'inscrire dans les cultures 
ministérielles, la qualité des évaluations reste inégale entre secteurs et l'évaluation environnementale n'est pas systématique (Gouvernement, 2018 ; France Stratégie, 2019).

\section{Graphique 2. Indicateurs de la gouvernance des infrastructures}

2016, indice variant sur une échelle allant de 0 (performance la moins bonne) à 100 (performance la meilleure)

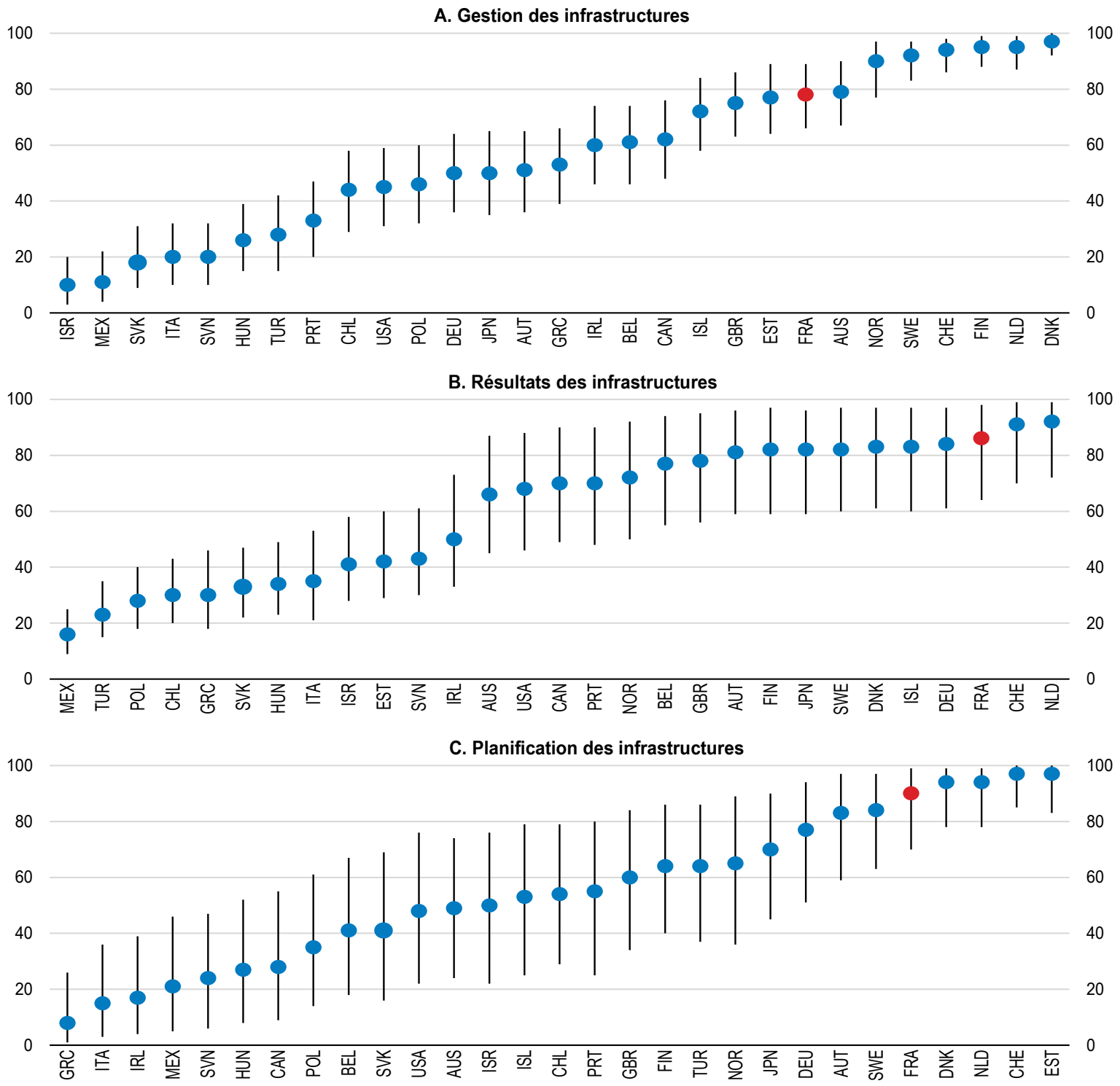

Note: Ces indicateurs de gouvernance permettent de mesurer les résultats obtenus par chaque pays dans chacune des trois dimensions suivantes : planification, gestion et résultats des infrastructures. Les cercles indiquent le score des pays, tandis que les lignes verticales correspondent aux incertitudes statistiques entourant ces résultats. Les indicateurs de gouvernance sont construits à partir des résultats d'une enquête auprès d'experts en infrastructures publiques.

Source: Hertie School of Governance (2016). The Governance Report 2016: Infrastructure Governance Indicators, Hertie School of Governance: Berlin.

StatLink ज्ञाs https://doi.org/10.1787/888933952670 


\section{Les collectivités locales sont le premier investisseur public, et le secteur privé pourrait jouer un rôle accru}

L'investissement public est une responsabilité partagée entre échelons administratifs, et ce sont les collectivités locales qui investissent le plus (Graphique 3). C'est donc d'elles que les politiques visant à accroître l'efficience de l'investissement public dépendent en grande partie. Les collectivités locales investissent dans un large éventail de secteur, tandis que les investissements réalisés par l'administration centrale concernent surtout les affaires économiques (notamment les transports), la défense et la santé (Graphique 4, partie A). En France, il existe trois niveaux d'administration locale : les régions, les départements et les communes. De plus, toutes les communes font partie d'un EPCI (établissement public de coopération intercommunale) qui, dans la pratique, représente un échelon supplémentaire d'administration locale se situant entre les communes et les départements. Le secteur communal représente l'essentiel des investissements des collectivités locales ; viennent ensuite les niveaux supérieurs qui, chacun, investissent environ le même montant (partie B).

\section{Graphique 3. Répartition des dépenses d'investissement entre les différents niveaux de l'administration}

En pourcentage, $2016^{1}$

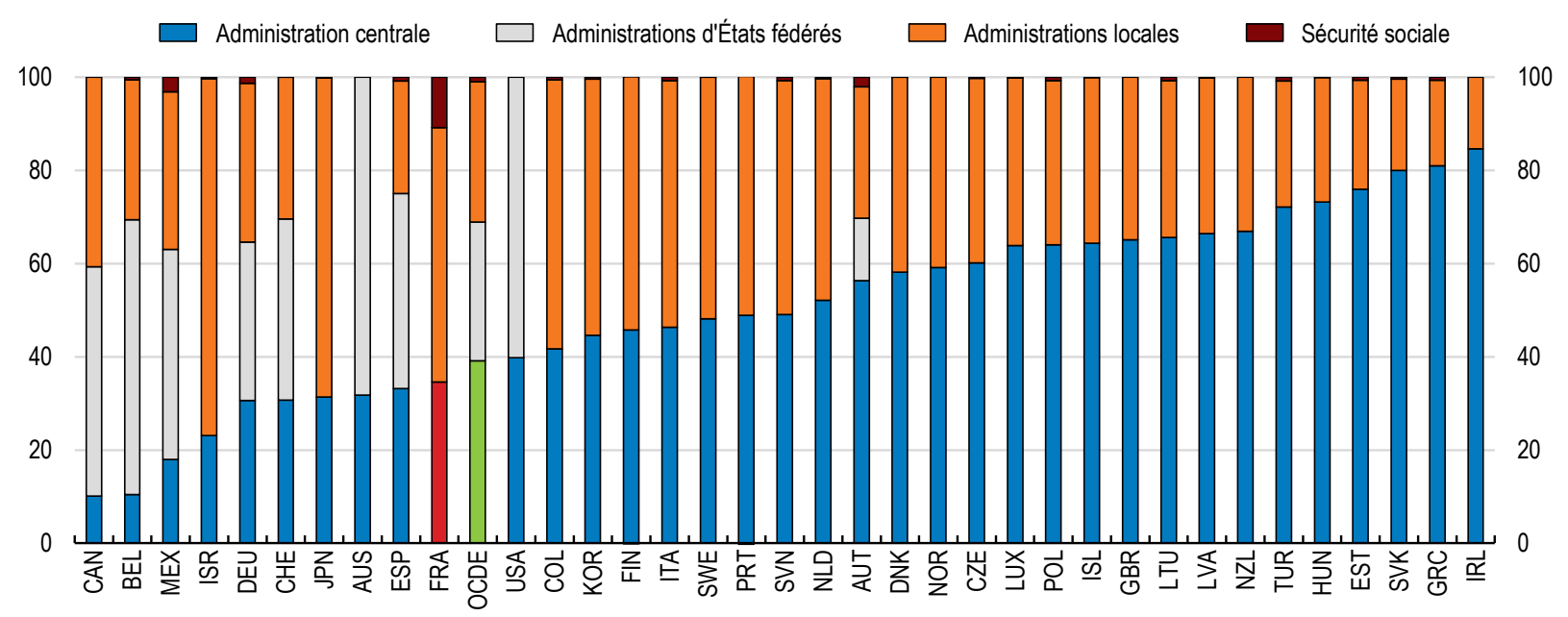

1. Ou dernière année disponible. Les administrations locales sont comptabilisées dans la catégorie des « administrations centrales » pour l'Australie et les États-Unis. En Australie, il n'existe pas de système public d'assurance sociale. Les administrations de sécurité sociale sont comptabilisées dans la catégorie « administration centrale » aux États-Unis, en Irlande, en Norvège, en Nouvelle-Zélande et au Royaume-Uni. Les administrations d'États fédérés n'existent que dans les neuf pays membres de l'OCDE dotés d'une structure fédérale : l'Allemagne, l'Australie, l'Autriche, la Belgique, le Canada, l'Espagne (pays considéré comme quasi-fédéral), les États-Unis, le Mexique et la Suisse.

Source: OCDE (2017), Panorama des administrations publiques (base de données).

StatLink त्गाड़ https://doi.org/10.1787/888933952689

La participation du secteur privé à la réalisation d'infrastructures augmente depuis 2004, date à laquelle les contrats de partenariat ont été mis en place. D'autres articulations publicprivé existent également comme pour les grands projets d'aménagement urbains récents. Les partenariats public-privé existent depuis longtemps en France dans les secteurs 
commerciaux; ils s'appuient sur un mode de financement des infrastructures par les usagers où le secteur privé est responsable de la gestion et de l'entretien des actifs. Toutefois, le recueil d'informations sur les résultats financiers et opérationnels des actifs selon les différents modèles de partenariats public-privé n'est pas satisfaisant (Saussier et Tirole, 2015), ce qui empêche de prendre des décisions plus éclairées sur l'implication du secteur privé dans la réalisation des infrastructures. En outre, étant donné les importants besoins de financement qu'il va falloir couvrir pour mettre au point des infrastructures bascarbone, il faudrait envisager de mieux mobiliser le secteur privé en identifiant clairement, dans les plans sectoriels à long terme, les possibilités d'investissement dans de telles infrastructures (voir ci-après).

\section{Graphique 4. Investissements des collectivités locales}
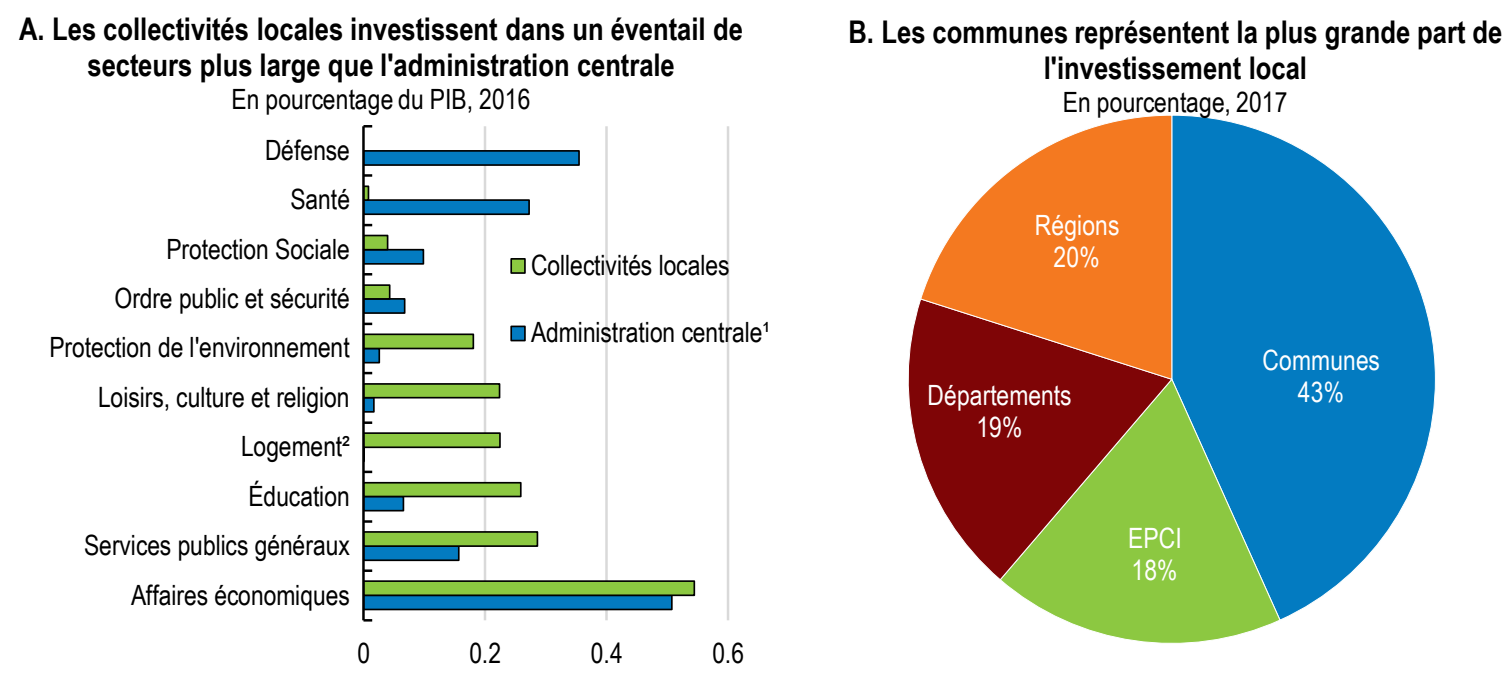

1. Y compris les dépenses de sécurité sociale

2. Et équipements collectifs

Note : Dans la partie B, les chiffres sont présentés en comptabilité budgétaire sans retraiter les flux croisés entre collectivités territoriales.

Source : OCDE (2018), Statistiques de l'OCDE sur les comptes nationaux (base de données); DGCL (2018), Les chiffres-clés des collectivités locales, Direction Générale des Collectivités Locales, Paris, https://www.collectivites-locales.gouv.fr/files/files/statistiques/brochures/chiffres_cles_2018.pdf.

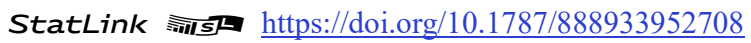

\section{Investir pour l'avenir}

L'investissement public devrait viser à atteindre les engagements nationaux et internationaux pris par la France dans le domaine de l'environnement, comme prévu dans le dernier plan d'investissement des autorités (Grand plan d'investissement, Encadré 2). Favoriser des modes de transport plus durables sera essentiel pour s'attaquer au problème de la pollution de l'air, qui contribue au changement climatique et entraîne des coûts considérables en matière de santé. La France est l'un des rares pays à avoir élaboré une stratégie à long terme de faible niveau d'émissions, et l'enjeu consiste à s'assurer que le soutien public à la transition environnementale soit correctement orienté en vue d'atteindre ces objectifs. Le rôle des collectivités territoriales sera crucial à cet égard car elles représentent l'essentiel des investissements liés à l'environnement et au climat en France (OCDE/Banque mondiale/ONU Environnement, 2018). Il sera également important de rendre les plans d'investissement dans les infrastructures de transport cohérents avec les 
objectifs de forte décarbonation afin d'éviter d'investir dans des infrastructures que la transition énergétique rendraient caduques. La révision de la valeur tutélaire du carbone prise en compte dans les évaluations des grands projets d'investissement va dans ce sens. Les autorités vont aussi devoir relever le défi consistant à ramener la part de l'énergie nucléaire à $50 \%$ dans la production d'électricité à moyen terme, tout en garantissant la sécurité de l'approvisionnement en énergie et en maintenant à de faibles niveaux l'empreinte carbone de la production d'énergie.

\section{Encadré 2. Le Grand plan d'investissement et le Fonds pour l'innovation et l'industrie}

Le Grand plan d'investissement (GPI), qui couvre la période 2018-22, est un dispositif interministériel sous la direction du Premier ministre qui vise à gagner en efficience et à disposer d'outils permettant un réel pilotage avec arbitrages. Le Secrétariat général pour l'investissement (SGPI) doit annuellement proposer, sur la base d'une évaluation de la mise en œuvre, des résultats et des impacts, l'analyse du plan dans son ensemble et préconiser les arbitrages. Le GPI intègre le dernier volet des programmes d'investissement d'avenir (PIA), et s'en inspire dans sa recherche d'efficacité.

Ce plan comporte 26 mesures regroupées en quatre grands axes prioritaires pour un investissement total de 57 milliards d'euros (soit 2,5\% du PIB de 2017). La première priorité, qui concerne la transition écologique, consiste à mettre l'accent sur l'amélioration de la situation énergétique des bâtiments, et à accélérer le déploiement des énergies renouvelables et des modes de transport durables (20 milliards d'euros). La deuxième consiste à investir dans les compétences en ciblant les chômeurs de longue durée et les jeunes qui ne sont ni en emploi, ni scolarisés, ni en formation, comme évoqué dans le chapitre 1 (15 milliards d'euros). L'innovation et l'enseignement supérieur font l'objet de la troisième priorité (13 milliards d'euros) : c'est dans cet axe que se trouvent les actions du PIA pour l'essentiel. Enfin, la dernière priorité vise principalement la transformation numérique des services publics et la modernisation des hôpitaux ( 9 milliards d'euros).

Différents ministères seront chargés de distribuer les crédits, et $3 \%$ des sommes sont mises de côté chaque année pour que les fonds soient affectés aux actions donnant les meilleurs résultats. Le Secrétariat général pour l'investissement assure la supervision générale du GPI, l'objectif étant de préserver les points forts qui étaient ceux des PIA comme l'importance donnée aux évaluations, la sélectivité dans l'allocation des ressources et la coopération public-privé (OCDE, 2014a). Le GPI est financé à hauteur d'environ 24 milliards d'euros par de nouvelles mesures avec impact budgétaire, tandis que le reste du programme est une intégration dans le dispositif de pilotage transversal interministériel de crédits déjà budgétés ministériellement, dans l'esprit du fonctionnement du COPERMO (Encadré 3).

Un nouveau fonds pour l'innovation (Fonds pour l'innovation et l'industrie, FII) a été créé en 2018 pour financer des innovations technologiques de rupture grâce à un soutien supplémentaire à destination des jeunes entreprises opérant dans le secteur des technologies de pointe à forte intensité capitalistique et par le biais d'une nouvelle initiative appelée "grands défis", qui financera des projets d'innovation de rupture à la frontière technologique pour répondre à un enjeu sociétal.

Source: Le Grand Plan d'Investissement 2018-2022, Rapport au Premier ministre, https://www.gouvernement.fr/action/le-grand-plan-d-investissement-2018-2022 et Séance inaugurale du Conseil de l'Innovation, https:/www.entreprises.gouv.fr/files/files/directions services/politique-etenjeux/innovation/dossier-presse-conseil-innovation.pdf. 
Une infrastructure numérique efficiente, fiable et largement accessible est indispensable pour tirer pleinement parti de la révolution numérique, et la généralisation du numérique jouera un rôle de premier plan pour améliorer l'efficience du secteur public. L'avènement du numérique et de ses variantes les plus avancées a le potentiel de réduire les coûts liés à la montée en gamme de la production et de stimuler les échanges, avec des retombées sur l'innovation, la diffusion de la technologie et la productivité. Dans ce contexte, les autorités ont pour objectif d'offrir l'accès à l'internet haut débit à tous les ménages, entreprises et administrations d'ici 2022, grâce à une combinaison d'investissements publics et privés (Plan France Très Haut Débit). Dans l'administration, les technologies numériques se développent, mais leur usage reste modeste par rapport à ce que l'on observe dans les pays les plus performants, ce qui pèse sur la capacité des autorités à se servir du numérique pour atteindre leurs objectifs budgétaires à moyen terme. Par exemple, l'investissement numérique dans l'administration fiscale est faible (Cour des comptes, 2018a), ce qui limite la capacité des autorités à réformer les services fiscaux. Cependant, le GPI vise également la transformation numérique des services publics.

La recherche-développement $(R \& D)$ est un moteur essentiel de la performance économique à long terme et sera indispensable pour affronter le problème du changement climatique. Les financements publics affectés à la R\&D sont comparables à la moyenne de l'OCDE, tandis que les crédits budgétaires publics de $R \& D$ ont plus significativement diminué depuis 2008 que dans la plupart des autres pays du G7 (Graphique 5). Comme d'autres pays de l'OCDE, les autorités françaises ont mis l'accent sur les incitations fiscales (notamment le crédit d'impôt recherche) et aides à la R\&D des entreprises (OCDE, 2018a). La R\&D privée a tendance à privilégier le développement par rapport à la recherche fondamentale. À cet égard, la R\&D privée stimule la compétitivité des entreprises, tandis que des travaux de recherche fondamentale moins directement ciblés sont aussi importants pour encourager l'innovation et relever les grands défis mondiaux, qu'ils soient sociaux ou environnementaux. Par ailleurs, des programmes d'investissement ponctuels bien conçus par les autorités françaises ont compensé de façon partielle la baisse des crédits publics récurrents alloués à la recherche, et le dernier plan d'investissement contient également des mesures bienvenues pour soutenir les meilleurs établissements universitaires de recherche (Encadré 2). 
Graphique 5. Le financement public de la recherche est proche de la moyenne OCDE

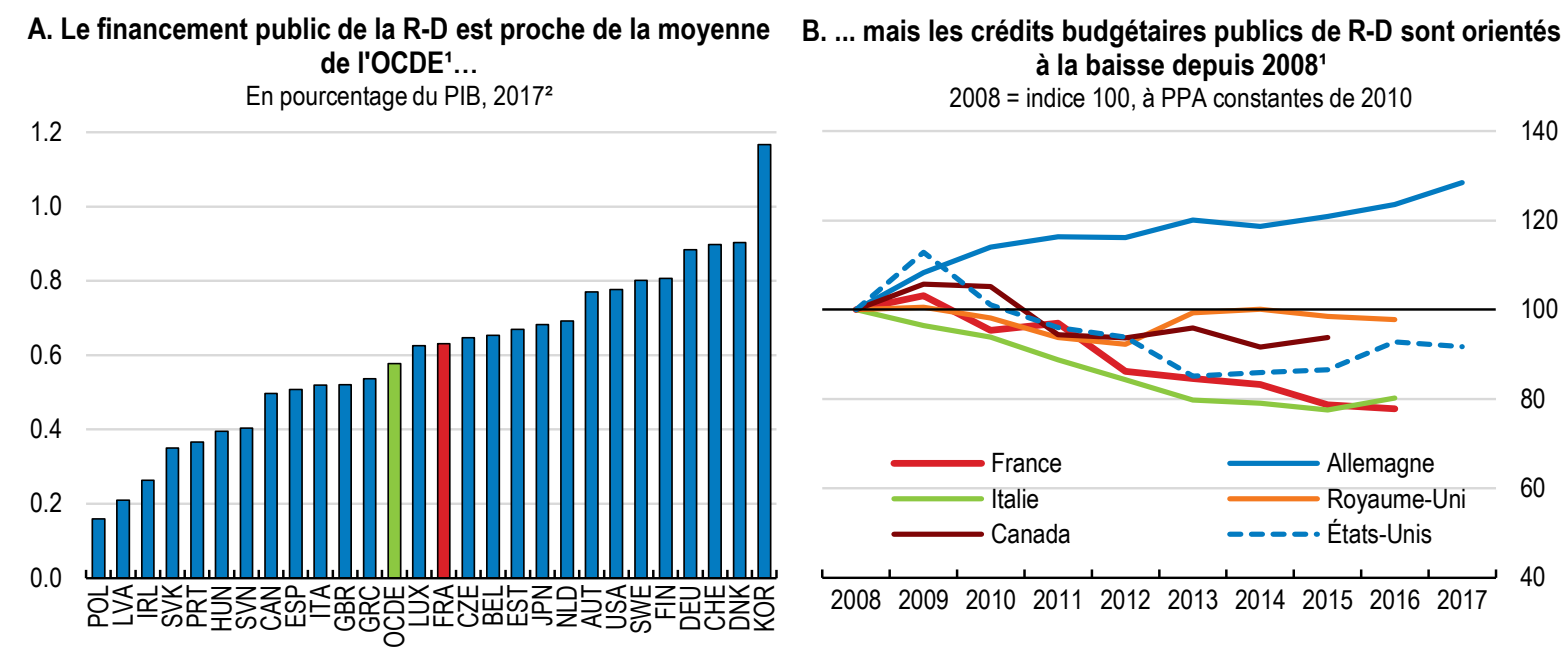

1. Les données représentées sont les crédits budgétaires publics de R\&D (CBPRD) hors incitations fiscales. Ces données diffèrent de la dépense intérieure de recherche et développement (DIRD) financée par l'administration qui indiquent que les dépenses de R\&D de l'enseignement supérieur et de l'État en France sont globalement stables par rapport au PIB depuis 2008. CBPRD et DIRD financée par l'administration diffèrent principalement à deux égards. Premièrement, les données DIRD sont fondées sur les déclarations des exécutants de la R\&D, alors que les données CBPRD s'appuient sur celles des bailleurs de fonds. Ainsi, la différence entre les données CBPRD et DIRD financée par l'administration pourrait venir du traitement budgétaire particulier des programmes d'investissement d'avenir (PIA). Deuxièmement, les séries fondées sur la DIRD ne couvrent que la $R \& D$ exécutée sur le territoire national, alors que celles fondées sur les CBPRD couvrent également les sommes versées à des exécutants étrangers.

2. Ou dernière année disponible.

Source : OCDE (2018), Statistiques de la recherche et développement (base de données).

StatLink त्गा5म https://doi.org/10.1787/888933952727

\section{Renforcer les conditions-cadres pour un investissement public efficace}

Les investissements de grande ampleur concernent différents secteurs et domaines d'action publique et ont des effets à long terme. Il en résulte un processus décisionnel complexe faisant intervenir différents niveaux d'administration et de multiples intervenants. L'OCDE a formulé des recommandations dans le but d'aider les pouvoirs publics à évaluer les forces et les faiblesses de leurs capacités en matière d'investissement public (Tableau 2), et l'OCDE assure le suivi de la mise en œuvre de ces recommandations (OCDE, 2018b). Partant de ces principes, le cadre de gouvernance des infrastructures de l'OCDE met en lumière les bonnes pratiques concernant l'investissement dans les infrastructures (OCDE, 2017b), notamment la nécessité de définir une vision stratégique quant aux besoins en infrastructures à long terme. 
Tableau 2. Principes de l'OCDE pour un investissement public efficace

\begin{tabular}{|c|c|}
\hline Piliers & Principes \\
\hline $\begin{array}{l}\text { Coordonner l'investissement public entre les différents } \\
\text { niveaux d'administration et les politiques publiques }\end{array}$ & $\begin{array}{l}\text { Investir en suivant une stratégie intégrée, adaptée aux } \\
\text { différents territoires } \\
\text { Adopter des instruments efficaces de coordination } \\
\text { entre les administrations, à l'échelon national et } \\
\text { infranational } \\
\text { Assurer une coordination horizontale entre les } \\
\text { administrations infranationales afin d'investir à } \\
\text { l'échelon pertinent }\end{array}$ \\
\hline $\begin{array}{l}\text { Renforcer les capacités en matière d'investissement } \\
\text { public et promouvoir la reproductibilité des politiques } \\
\text { publiques à tous les échelons de l'administration }\end{array}$ & $\begin{array}{l}\text { Évaluer en amont les effets à long terme de } \\
\text { l'investissement public et les risques connexes } \\
\text { Impliquer les parties prenantes tout au long du cycle } \\
\text { d'investissement } \\
\text { Mobiliser les acteurs et institutions de financement } \\
\text { privés pour diversifier les sources de financement et } \\
\text { consolider les capacités } \\
\text { Renforcer l'expertise des agents et des institutions } \\
\text { publics concernés par les projets d'investissement } \\
\text { public } \\
\text { Mettre l'accent sur les résultats et favoriser } \\
\text { l'apprentissage tiré de l'expérience }\end{array}$ \\
\hline $\begin{array}{l}\text { Veiller à l'existence d'un cadre d'investissement public } \\
\text { solide à tous les niveaux de l'administration }\end{array}$ & $\begin{array}{l}\text { Mettre en place un cadre budgétaire adapté aux } \\
\text { objectifs d'investissement recherchés } \\
\text { Développer une gestion financière solide et } \\
\text { transparente à tous les échelons de l'administration } \\
\text { Promouvoir la transparence de la commande publique } \\
\text { et son utilisation stratégique à tous les niveaux de } \\
\text { l'administration } \\
\text { Veiller à la qualité et à la cohérence de la } \\
\text { réglementation à tous les échelons de l'administration }\end{array}$ \\
\hline
\end{tabular}

Source: Recommandation du Conseil de l'OCDE pour un investissement public efficace entre niveaux de gouvernement (OCDE, 2014b).

\section{Vers une meilleure hiérarchisation et une meilleure sélection des projets d'investissement}

\section{Promouvoir le recours à des évaluations socio-économiques avant les décisions d'investissement}

Pour aider à hiérarchiser les projets d'investissement, la France a introduit en 2013 des exigences relatives aux évaluations socio-économiques pour toutes les opérations d'investissement public, à l'exception des projets concernant le secteur de la défense et de ceux financés en totalité par les collectivités locales. Les projets d'investissement dont le financement par l'État ou ses opérateurs dépasse le seuil de 20 millions d'euros doivent notamment faire l'objet d'une évaluation socio-économique ex ante, et être regroupés au sein d'un inventaire afin d'en assurer un suivi cohérent entre les différents secteurs. En outre, une contre-expertise indépendante de l'évaluation ex ante conduite par le Secrétariat général pour l'investissement (SGPI) est obligatoire pour les projets dont la valeur dépasse 100 millions d'euros. Sur la base de ce rapport de contre-expertise, un avis simple est rendu par le SGPI. L'ensemble est diffusé aux différentes parties prenantes de l'Etat et au Parlement. Il doit également faire partie du dossier d'enquête publique le cas échéant et doit être considéré plus généralement avant la décision finale. 
Bien que les procédures d'évaluation des projets d'investissement public varient d'un pays à l'autre, ce seuil de 100 millions d'euros déclenchant l'obligation d'une contre-expertise semble quelque peu élevé au regard d'autres pays de l'OCDE. En Norvège, par exemple, les projets d'investissement public dans les infrastructures de transport doivent faire l'objet d'un contrôle de qualité dès lors qu'ils sont supérieurs à 750 millions de couronnes norvégiennes. En Australie, Infrastructure Australia, un organisme public, conduit des analyses coûts-bénéfices lorsque les projets d'infrastructure excèdent 100 millions de dollars australiens (ce qui est au-dessus du seuil français de 20 millions d'euros et inférieur à celui de 100 millions d'euros pour le déclenchement d'une contre-expertise).

Le nombre de contre-expertises varie d'une année sur l'autre. Les avis rendus (60 depuis 2013), même favorables (26 depuis 2013), sont le plus souvent assortis de recommandations issues de la contre-expertise. Deux avis défavorables ont été rendus depuis 2013. Lorsque le dossier d'évaluation du porteur de projet s'avère insuffisant pour éclairer la décision publique, l'avis du SGPI émet des réserves qui ont parfois conduit à des reconfigurations de projets. Sectoriellement, le transport représente moins d'un quart des projets contre-expertisés et les hôpitaux un tiers.

Afin de déterminer des éléments de doctrine pour les analyses socio-économiques et de fournir aux porteurs de projets des outils normés (tableurs, valeurs tutélaires, scénarios macroéconomiques ...), un groupe d'experts a été installé en 2016. Cette initiative se justifie d'autant plus que l'absence de méthodes bien définies dans certains secteurs a constitué un obstacle à l'évaluation socio-économique des grands projets d'investissement (Inspection générale des Finances, 2016). Il convient d'encourager et de renforcer les mesures destinées à développer des méthodes d'évaluation des projets d'investissement, et de promouvoir davantage le recours aux analyses socio-économiques lors de la programmation des investissements, en établissant une stratégie en vue de toucher toutes les parties prenantes concernées.

Toutefois, malgré la présence de dispositifs réglementaires solides de sélection des projets d'investissement, les considérations d'efficience économique n'ont souvent qu'une influence limitée sur le choix effectif des projets. Il peut arriver en particulier que la sélection des projets réponde à des pressions exercées par les parties intéressées, cependant que la gouvernance des infrastructures obéit souvent à des considérations d'ordre politique. Par exemple, le réseau ferroviaire à grande vitesse est bien développé en France, mais compte un nombre de gares qui semble excessif (on en dénombre plus de 230 capables d'accueillir des trains à grande vitesse), et toutes les lignes à grande vitesse ne sont pas des investissements rentables (Cour des comptes, 2014). Les considérations d'efficience économique interviennent également rarement dans les décisions de maintenir l'exploitation de lignes ferroviaires peu empruntées (Spinetta, 2018).

Si l'investissement public relève, in fine, d'un choix politique, exiger des autorités qu'elles justifient, formellement et publiquement, leurs décisions lorsque celles-ci ne coïncident pas avec les résultats de la contre-expertise dans le cas des grands projets d'investissement, contribuerait à améliorer la transparence et à renforcer le rôle des analyses socioéconomiques dans la sélection des projets d'investissement. Il faudrait pour cela publier systématiquement les contre-expertises des évaluations ex ante conduites par le SGPI, ce qui n'est pas la pratique actuelle bien que la plupart des rapports de contre-expertise soient finalement publiés. Les autorités pourraient également envisager de dissocier l'activité de contre-expertise du SGPI, organisme placé sous l'autorité du Premier ministre et chargé également de la promotion des investissements. Cette dissociation permettrait d'éviter tout conflit d'intérêt qui pourrait survenir dans le cadre institutionnel actuel. En Australie, 
Infrastructure Australia, l'organisme chargé de la planification, bénéficie du soutien des acteurs intéressés et de tous les partis politiques. Cette institution indépendante est responsable de la publication des analyses techniques que les autorités sont officiellement tenues d'examiner avant de rendre leur décision concernant les grands projets d'infrastructure.

\section{Diffuser les bonnes pratiques auprès de tous les acteurs concernés}

Les procédures de sélection des projets d'investissement et le recours aux évaluations socio-économiques ex ante varient considérablement d'un ministère à l'autre. Il était donc nécessaire de mettre en place dans chaque ministère des processus décisionnels et d'analyse qui soient adaptés au cycle de vie de tous les projets d'investissement de grande envergure. Cette initiative permet de mieux identifier les projets prioritaires et d'accélérer la diffusion des meilleures pratiques applicables aux évaluations ex ante. Au Royaume-Uni, on peut noter que les principes qui régissent les investissements publics sont regroupés dans le Green Book. La mise en œuvre des projets d'investissement repose sur un processus de validation par étapes, dites "gateway reviews", destiné à vérifier leur adéquation stratégique, leur rentabilité économique, leur faisabilité financière, leur validité commerciale et les modalités de gestion des avantages attendus (HM Treasury, 2015). En 2017, un guide similaire (Guide de l'évaluation socioéconomique des investissements publics) a été publié en France par France Stratégie et la Direction générale du Trésor.

Afin d'homogénéiser les procédures de sélection des investissements, la France pourrait s'inspirer du cadre existant dans le secteur de la santé pour tous les secteurs concernés par des projets d'investissement de grande ampleur. Depuis 2013, le Comité interministériel de performance et de la modernisation de l'offre de soins hospitaliers (COPERMO) supervise en effet la totalité des grands projets d'investissement hospitalier du pays et intervient à différents stades du cycle d'investissement (Encadré 3). Il réalise notamment une évaluation des analyses coûts-bénéfices, et peut demander aux porteurs de projet, à la lumière de ses conclusions, de retravailler les projets. L'évaluation ex ante des projets d'investissement hospitalier fait également l'objet d'une contre-expertise par le SGPI, qui est membre à part entière du COPERMO ; le SGPI assure ainsi un niveau de contrôle supplémentaire quant à la qualité des évaluations, lorsque le financement public du projet dépasse 100 millions d'euros. 


\section{Encadré 3. Processus décisionnel des grands projets d'investissement hospitalier}

Malgré la baisse enregistrée depuis 2013, du fait de l'achèvement des plans d'investissement exceptionnels visant à moderniser les hôpitaux (plans Hôpital 2007 et 2012), les investissements publics dans le secteur de la santé restent importants et s'élèvent à 6.2 milliards d'euros en $2016(0.3 \%$ du PIB $)$, dont $80 \%$ d'investissements hospitaliers. Le Comité interministériel de performance et de la modernisation de l'offre de soins (COPERMO) a été mis en place en 2013 par le ministère de la Santé. Il s'agit d'un dispositif chargé du contrôle et de l'évaluation des investissements hospitaliers de grande envergure, suite à la mise en place de nouvelles exigences relatives aux évaluations socio-économiques des grands investissements; il doit aussi permettre de mieux hiérarchiser les investissements. Le COPERMO se compose de représentants des ministères de la Santé, du SGPI et du Budget. Il intervient à deux reprises dans le processus décisionnel de l'ensemble des projets d'investissement hospitalier supérieurs à 50 millions d'euros.

- Au démarrage d'un projet, l'établissement ou l'Agence régionale de santé (ARS) soumet la proposition d'investissement au COPERMO en vue de son éligibilité, ce qui constitue une première occasion de recueillir des commentaires sur l'opération envisagée ;

- Le COPERMO intervient ensuite au stade de la validation du mécanisme de financement avant que le ministère de la Santé ne rende une décision quant au montant de sa participation financière.

Lors de chacune de ces deux étapes, le COPERMO peut demander que soient apportées des modifications au projet ou bien en reporter l'examen jusqu'à ce que les ajustements requis aient été appliqués. Un inventaire annuel des projets d'investissement est ensuite réalisé afin d'assurer un suivi des projets en cours, ainsi que des recommandations formulées par le COPERMO.

Le ministère de la Santé élabore également des notes méthodologiques - qui s'appuient dans une large mesure sur les retours d'expérience du COPERMO - afin de diffuser les bonnes pratiques en matière d'efficience des investissements hospitaliers auprès des acteurs concernés.

Source : (Inspection générale des Finances, 2016) et ministère des Solidarités et de la Santé (https://solidaritessante.gouv.fr/professionnels/gerer-un-etablissement-de-sante-medico-social/performance-des-etablissementsde-sante/efficience-hospitaliere).

Imposer une évaluation ex post des grands projets d'investissement dans tous les secteurs permettrait d'éclairer utilement les futures décisions d'investissement. Les évaluations $e x$ post sont obligatoires depuis 1982 dans le secteur des transports, où la comparaison des résultats ex post des projets au regard de leurs objectifs ex ante est obligatoire. Cette obligation vise les projets de transport d'un montant supérieur à 83.1 millions d'euros, et l'évaluation ex post doit avoir lieu entre trois et cinq ans après l'achèvement du projet. Le fait de soumettre tous les secteurs à cette règle - par exemple, pour les investissements supérieurs à 100 millions d'euros comme dans le cas du contrôle de la qualité des évaluations socio-économiques ex ante réalisées par le SGPI - contribuerait à améliorer la qualité des évaluations ex ante à l'avenir. 
Il peut s'avérer compliquer de mesurer les effets d'additionnalité des investissements, c'està-dire de ne pas se contenter d'évaluer la réussite d'un projet uniquement à l'aune du respect des délais et du budget impartis. Par exemple, on observe que les avantages découlant des projets d'infrastructure de transport pour l'économie, en termes d'emploi, reposaient souvent sur de simples analyses de corrélation (CEREMA, 2018). Dans ce cas, il peut s'avérer nécessaire de s'appuyer sur l'expertise d'organismes indépendants ayant la capacité d'effectuer des évaluations de grande qualité.

Les opérations d'investissement de grande envergure des collectivités locales doivent être soumises aux mêmes obligations que celles de l'administration centrale. L'essentiel des investissements public est réalisé par les collectivités locales, mais la planification des opérations d'investissement varie considérablement d'une collectivité locale à l'autre, même entre les plus importantes d'entre elles (Cour des comptes, 2015a). Les résultats des enquêtes montrent en outre que la France est l'un des pays de l'UE où les communes ont tendance à peu recourir aux évaluations ex ante des projets d'infrastructure (Banque européenne d'investissement, 2017). La loi de 2015 portant nouvelle organisation territoriale de la République (NOTRe) prévoit que toutes les opérations exceptionnelles d'investissement des collectivités locales-dans la pratique, celles supérieures à 5 millions d'euros - doivent faire l'objet d'une étude relative à l'impact des opérations concernées sur les dépenses de fonctionnement à venir. Cette évaluation est obligatoire pour les projets d'investissement supérieurs à 100 millions d'euros. Or, ces études d'impact ne constituent pas à proprement parler des analyses socio-économiques, permettant d'évaluer tout l'éventail des avantages et des coûts d'un projet d'investissement, à moins que son cofinancement par l'État ne dépasse 20 millions d'euros.

Il serait souhaitable, par conséquent, de soumettre les investissements des collectivités et des entreprises publiques locales aux mêmes obligations en matière d'analyses socioéconomiques que celles qui s'appliquent à l'État et ses opérateurs, notamment l'obligation de conduire une contre-expertise de l'évaluation ex ante pour les investissements dont le financement public est supérieur à 100 millions d'euros. Cela renforcerait les arguments en faveur d'une dissociation de l'activité de contre-expertise du SGPI pour neutraliser les inquiétudes des collectivités territoriales concernant leur autonomie vis-à-vis de l'Etat. De plus, il faudrait élaborer des lignes directrices adaptées aux besoins des collectivités locales en matière d'évaluation socio-économique. Le Danemark est un exemple de pays de l'OCDE qui a mis au point un tableur facile à utiliser pour effectuer des évaluations socioéconomiques des projets de transport.

\section{Sensibiliser le grand public aux considérations d'efficience économique afin d'améliorer les décisions d'investissement}

Il est nécessaire de sensibiliser l'opinion publique à la notion d'efficience économique des projets d'investissement, car l'opinion publique joue souvent un rôle important dans la sélection et la mise en œuvre des projets. Les grands projets d'urbanisme doivent faire l'objet d'une enquête publique qui vise à mieux informer la population sur les projets d'investissement programmés, mais aussi à permettre aux autorités de recueillir, auprès de tiers, des renseignements sur les risques, notamment environnementaux, que présente tel ou tel projet. À cet égard, recourir plus largement à des moyens de communication électroniques dans le cadre des enquêtes publiques, comme le prévoit la loi de 2010 portant engagement national pour l'environnement, contribuerait à sensibiliser davantage le grand public aux questions de rentabilité des projets d'investissement tant que la qualité des enquêtes publiques n'est pas affaiblie. Certaines collectivités locales, comme Bordeaux Métropole, diffusent ainsi systématiquement sur des sites internet dédiés, des informations 
détaillées sur les projets d'investissement nécessitant une enquête publique. Ces exemples mériteraient d'être plus largement suivis.

La participation des médias et de la société civile constitue un moyen privilégié de mieux sensibiliser la population au principe de l'efficience économique. Pour sensibiliser davantage l'opinion publique à la notion d'efficience économique des projets d'investissement, en renforçant le critère qualité-prix dans la sélection et l'exécution des projets d'investissement public, la Norvège, par exemple, a fait le choix de formations axées sur l'analyse coûts-bénéfices, dispensées aux journalistes économiques par l'administration.

\section{Tirer parti du secteur privé pour la réalisation des infrastructures}

La plupart des projets d'infrastructure font appel à une procédure standard de passation des marchés publics (maîtrise d'ouvrage publique). La participation du secteur privé à la mise en place d'infrastructures est cependant possible depuis 2004, date de l'introduction en France de contrats globaux avec financement public (partenariats public-privé (PPP), ou marchés de partenariat). Des contrats de PPP («marchés de partenariat ») sont conclus dans un grand nombre de secteurs, dont la santé, l'éducation, les installations sportives, la défense et les transports (Bergère, 2016).

Les partenariats public-privé, au sens large, existent depuis de nombreuses années en France dans les secteurs marchands, sous la forme d'un financement des infrastructures assuré par les usagers, la gestion et l'entretien des installations étant pris en charge par les opérateurs privés. C'est le cas par exemple dans le domaine de la distribution d'eau, où le secteur privé desservait $61 \%$ de la population en 2014 (Observatoire des services publics d'eau et d'assainissement, 2017), ou dans celui des autoroutes, avec $80 \%$ environ du réseau concédé à des entreprises privées. L'ouverture des marchés à la concurrence dans certains secteurs, comme celui des télécommunications, a également renforcé le rôle des entreprises privées dans le financement des infrastructures. Cependant, de manière générale, le stock de capital financé par le biais de PPP n'est pas très important rapporté au PIB (Graphique 6). 


\section{Graphique 6. Le stock de capital financé par le biais de PPP est plutôt limité}

En pourcentage du PIB, 2015

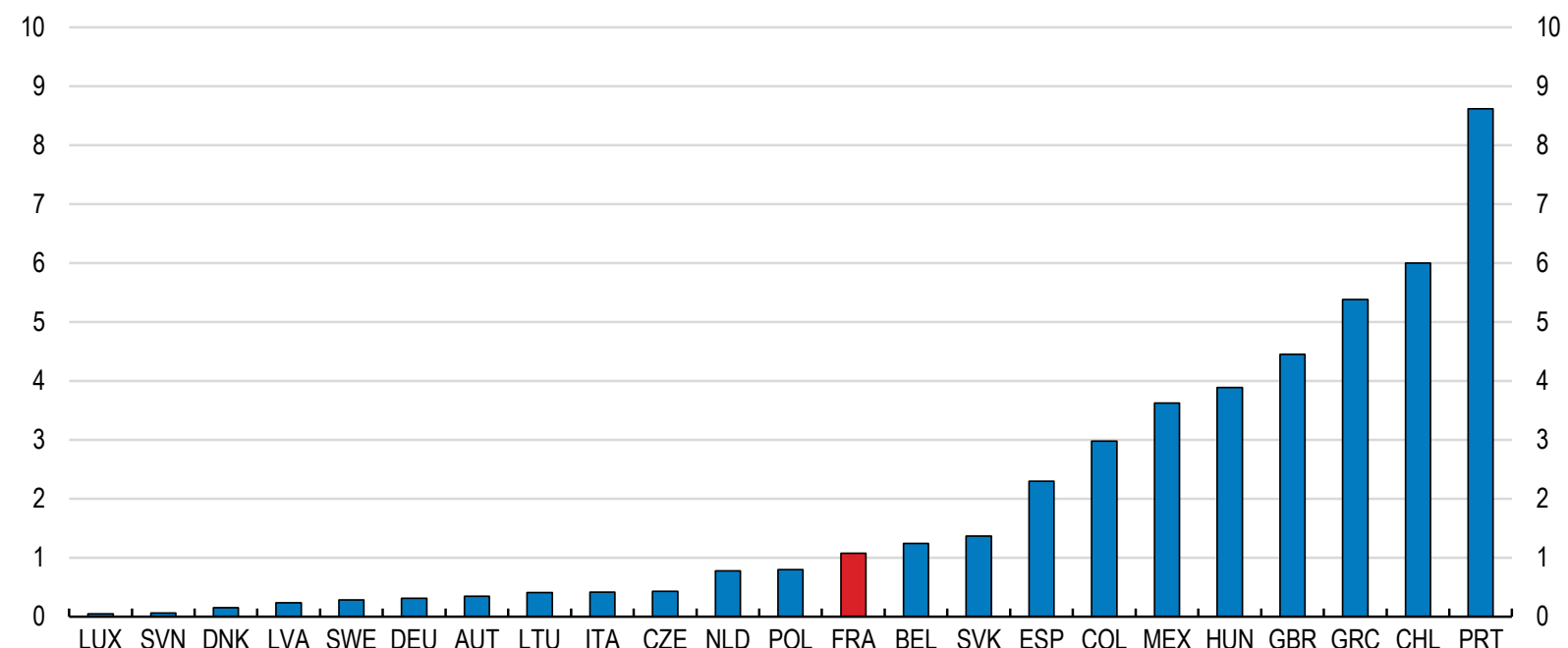

1. Ou dernière année pour laquelle des données sont disponibles. En ce qui concerne les pays européens, le stock de capital financé par le biais de PPP est mesuré sur la base des données de la Banque européenne d'investissement, qui recouvrent également des accords de concession comportant une opération de construction, la fourniture d'un service public et un véritable partage des risques entre le secteur public et le secteur privé pour les transactions dépassant 10 millions d'euros.

Source: FMI (2017), Investment and Capital Stock Statistics (base de données), Fonds monétaire international, Washington D.C.

StatLink त्गाडs https://doi.org/10.1787/888933952746

La constitution d'une liste de projets d'infrastructure en attente de financement contribuerait à attirer des investisseurs privés. C'est d'autant plus important pour répondre aux objectifs d'atténuation du changement climatique qui nécessiteront une réorientation radicale des investissements en vue de déployer des infrastructures pour la transition énergétique et la réduction des émissions de $\mathrm{CO} 2$. Les investissements annuels en infrastructures nécessaires d'ici à 2030 pour atteindre les objectifs économiques et de développement, et limiter le réchauffement climatique à moins de 2 degrés sont estimés à 6900 milliards USD dans le monde, soit environ $10 \%$ de plus que dans un scénario ne prévoyant aucune mesure de lutte contre le changement climatique (OCDE, 2017c). Les investissements entraînant une forte hausse des émissions de carbone devront notamment être réduits au profit de projets en lien avec l'efficacité énergétique et les énergies décarbonées. Bien souvent, ce n'est pas le manque de capitaux qui freine les investissements à l'appui des objectifs climatiques à long terme, mais plutôt le manque de projets pouvant être financés ou en attente d'investissement, auxquels les investisseurs privés et les promoteurs de projets peuvent consacrer du temps, des moyens et des ressources (OCDE, 2018c). À cet égard, la France pourrait faire davantage pour identifier précisément les possibilités d'investissement dans des infrastructures pour la transition énergétique et la réduction des émissions de $\mathrm{CO} 2$, dans le cadre de plans sectoriels à long terme (voir Section 3). Le Royaume-Uni, par exemple, s'est doté d'un cadre institutionnel lui permettant de recenser des projets prêts à être financés par l'intermédiaire de l'Infrastructure and Projects Authority (IPA), laquelle établit une liste de projets prioritaires afin de répondre aux besoins d'infrastructures à long terme du pays. 
La sélection des partenariats public-privé doit reposer sur des considérations d'efficience économique des projets. Il ne devrait y avoir aucun parti-pris d'ordre institutionnel, procédural ou comptable en faveur ou en défaveur des partenariats public-privé et la décision d'investir devrait être indépendante des modalités de montage et de financement du projet (OCDE, 2017d). Les PPP ne sont pas sans risque, dans la mesure où une mauvaise gestion peut donner lieu à des passifs éventuels pour les finances publiques, comme cela a été le cas au Portugal (Araújo and Sutherland, 2010). Ce sont des outils dont le pilotage peut s'avérer délicat, en particulier pour les collectivités locales qui ne disposent pas toujours des capacités administratives pour les utiliser efficacement (Sénat, 2014). Un décret de 2016 a d'ailleurs quelque peu limité le recours aux marchés de partenariat, en instaurant des seuils minimums pour pouvoir y recourir et en limitant le nombre d'opérateurs publics autorisés à utiliser ce type de contrats de PPP directement.

Utilisés à bon escient, les partenariats public-privé peuvent cependant aider les autorités à utiliser au mieux le savoir-faire du secteur privé pour la mise en place de services publics, comprenant à la fois la construction et l'entretien à long terme d'infrastructures complexes nécessitant des connaissances et des compétences techniques spécifiques. Les analyses du Forum International des Transports précisent les conditions à partir desquelles l'un des deux grands modèles de financement privé est le plus approprié en fonction de l'actif et du marché considérés (Forum international des transports, 2018). D'une part, selon le modèle d'actifs réglementés (regulatory asset base, RAB), il existe des négociations périodiques entre l'opérateur privé et un régulateur économique basées sur des objectifs d'efficacité. D'autre part, dans un modèle de PPP, le prix de l'infrastructure et les incitations à l'efficacité découlent de la concurrence pour le contrat ou la concession. L'aéroport d'Heathrow est un exemple de réglementation d'infrastructure basée sur les incitations (RAB) gérée par l'autorité de l'aviation civile du Royaume-Uni. C'est lorsque l'opérateur privé n'est pas soumis à la concurrence que le modèle $\mathrm{RAB}$ est généralement préférable. En outre, dans le cadre des contrats de régulation économique des aéroports, il semble justifié de bien définir l'ensemble des règles régissant le suivi et la surveillance de l'opérateur privé (Cour des comptes, 2018b).

Il serait souhaitable d'améliorer l'accès du grand public et des investisseurs à des données comparables sur la performance des projets d'infrastructure, quelles que soient les modalités de mise en œuvre, et de renforcer l'expertise des fonctionnaires qui participent à la sélection des marchés publics. Fin Infra, un service rattaché au ministère des Finances, est notamment chargé d'apporter conseils et appui technique sur tous les types de contrats complexes public-privé. Plusieurs ministères, comme celui des Transports par exemple, disposent aussi d'unités spécialisées dans les PPP. Cependant, les ressources affectées à la programmation des projets d'infrastructure mis en ouvre par le biais de PPP n'ont pas toujours été suffisantes par le passé, il semble donc nécessaire d'assurer un renforcement de l'expertise des agents du secteur public concernés par les PPP (Cour des comptes, 2015b; Saussier, 2017). En outre, améliorer l'accès du public et des investisseurs à des données comparables sur la performance des projets d'infrastructure, indépendamment des modalités de mise en œuvre, pourrait être obtenu en créant une agence chargée de collecter ces données. Une telle agence pourrait également conseiller les collectivités locales sur leurs choix en matière de marchés publics et assurer le suivi des performances des PPP une fois ceux-ci signés (OCDE, 2018c). Des données plus complètes sur les performances passées des investissements d'infrastructure permettraient également aux investisseurs de mieux calibrer la tarification des risques, ce qui pourrait, in fine, faire baisser le coût total des projets (Forum international des transports, 2018). 


\section{Renforcer les capacités des collectivités locales en matière d'investissement public}

\section{La poursuite des réformes institutionnelles contribuerait à dégager des gains d'efficience}

La France est l'un des pays de l'OCDE qui compte le plus grand nombre de collectivités locales (Graphique 7, partie A). La loi relative à la délimitation des régions, aux élections régionales et départementales et modifiant le calendrier électoral de 2015 s'est traduite par une diminution du nombre des régions, de 22 à 13 , sur le territoire métropolitain. Le nombre des intercommunalités a aussi été divisé par près de deux au cours des années qui ont suivi, pour s'établir à 1263 en 2018, notamment avec la création des métropoles, dont le périmètre couvre l'aire métropolitaine. La loi portant nouvelle organisation territoriale de la République (loi NOTRe) de 2015 est venue par ailleurs préciser les responsabilités des différents niveaux de collectivités. La France se distingue cependant des autres pays de l'OCDE par la petite taille de ses communes. Plus de la moitié d'entre elles comptent ainsi moins de 500 habitants, environ $85 \%$ des communes ont moins de 2000 habitants, et la taille médiane des communes, en nombre d'habitants, est la deuxième plus petite de l'OCDE (partie B).

\section{Graphique 7. Une fragmentation territoriale importante}
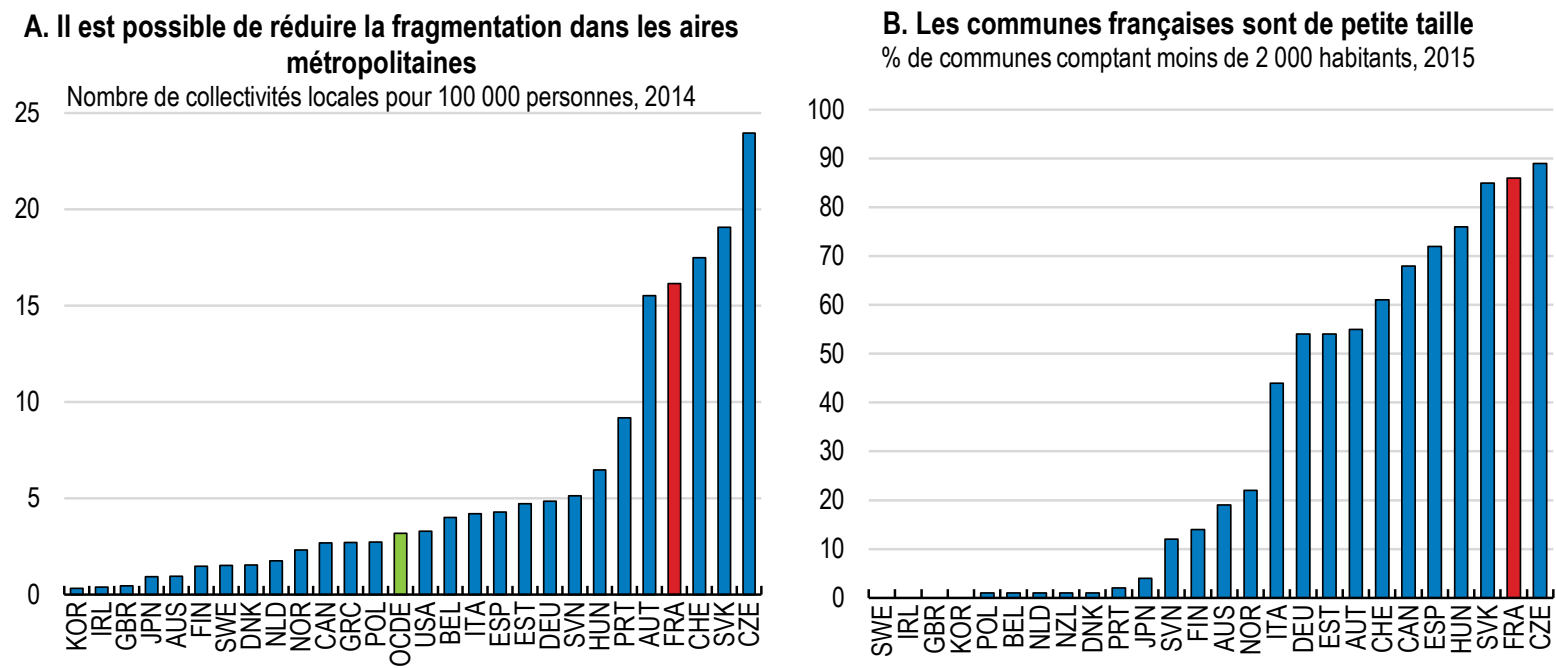

Source : OCDE (2015), Statistiques sur les aires métropolitaines (base de données); OCDE (2017), Finance et structure des gouvernements infranationaux (base de données).

\section{StatLink त्ञाज https://doi.org/10.1787/888933952765}

L'investissement des communes est d'une manière générale plus élevé dans les zones de montagne et les zones touristiques, ce qui tient aux écarts de coûts des biens et des services collectifs dans ces zones (Graphique 8, partie A). Toutefois, il n'existe pas de données consolidées relatives à l'investissement communal-c'est-à-dire tenant compte des investissements des échelons administratifs supérieurs dans les communes-ce qui complique l'analyse des disparités géographiques de l'investissement au niveau municipal. L'investissement par habitant des communes présente également un profil en $\mathrm{U}$, en ce sens qu'il est plus important dans les communes les plus petites et dans les plus grandes 
(Tableau 3), ce qui ne s'explique que partiellement par le caractère montagneux ou touristique de ces communes (partie B). Alors que les dépenses des collectivités locales présentent fréquemment un profil en U dans les pays de l'OCDE (OCDE, 2013), la France se caractérise par un grand nombre de communes de petite taille, ce qui suggère qu'une diminution de la fragmentation administrative permettrait de réaliser des économies d'échelle en matière d'investissement pour les communes les plus petites.

\section{Graphique 8. Dépenses d'investissement des communes par type de municipalités}
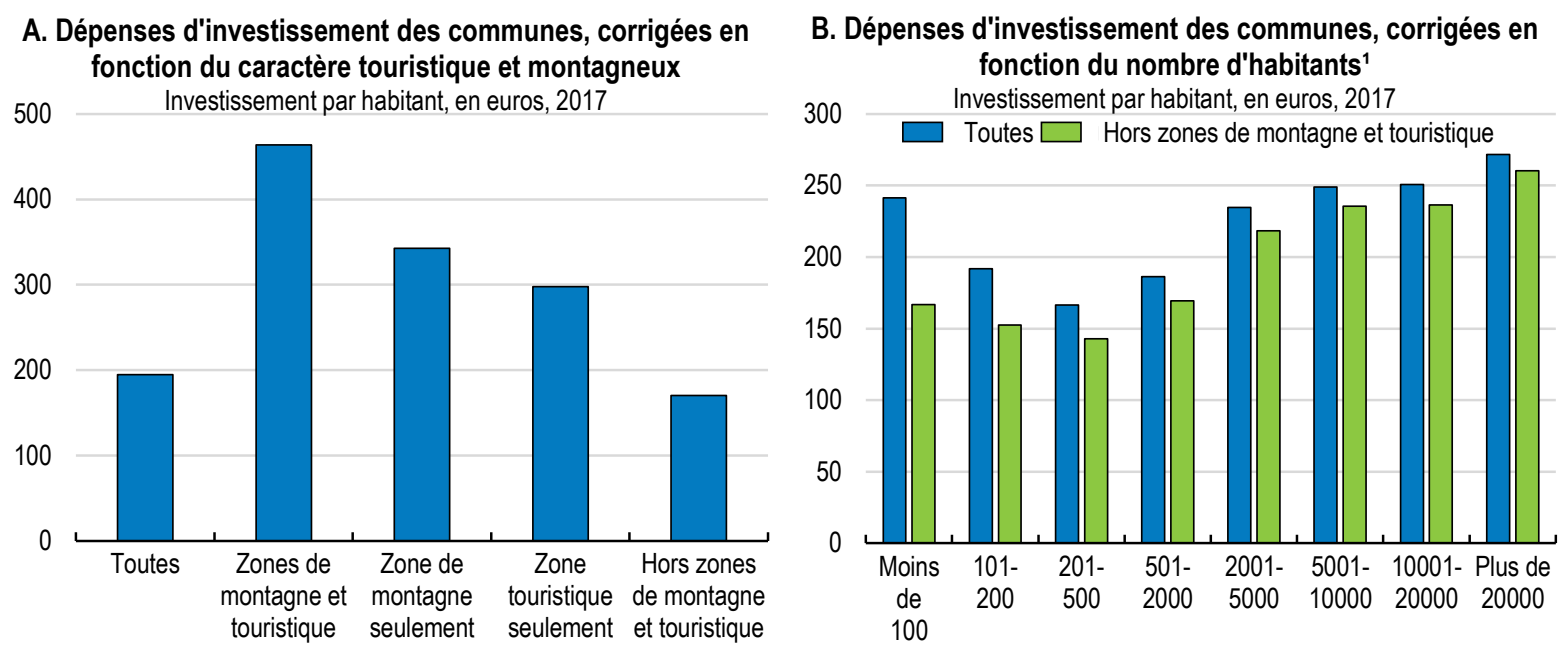

1. Les dépenses d'investissement des communes n'incluent pas l'investissement des intercommunalités. En supposant que les communes les plus petites tendent à déléguer relativement plus leurs opérations d'investissement à leur intercommunalité que les communes plus grandes, la partie B présenterait donc une estimation prudente de l'investissement excessif réalisé par les plus petites communes. Les dépenses d'investissement des communes sont indiquées pour toutes les communes et pour les communes qui ne sont situées ni en zone de montagne ni en zone touristique, car le nombre de communes appartenant aux autres catégories est trop faible pour certaines strates de population.

Source: L'Observatoire des finances et de la gestion publique locales, https://www.collectiviteslocales.gouv.fr/ofgl

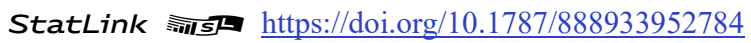

Tableau 3. Investissements des communes

Investissement communal médian par habitant, 2017

\begin{tabular}{|c|c|c|c|c|c|c|c|c|}
\hline $\begin{array}{l}\text { Taille des communes } \\
\text { (nombre d'habitants) }\end{array}$ & $\begin{array}{l}\text { Moins } \\
\text { de } 100\end{array}$ & $\begin{array}{l}{[101-} \\
200]\end{array}$ & $\begin{array}{l}{[201-} \\
500]\end{array}$ & $\begin{array}{l}{[501-} \\
2000]\end{array}$ & $\begin{array}{l}{[2001-} \\
5000]\end{array}$ & $\begin{array}{l}{[5001-} \\
10000]\end{array}$ & $\begin{array}{l}{[10001-} \\
20000]\end{array}$ & $\begin{array}{l}\text { Plus de } \\
20000\end{array}$ \\
\hline $\begin{array}{l}\text { Dépenses } \\
\text { d'investissement par } \\
\text { habitant }\end{array}$ & 241.4 & 191.9 & 166.5 & 186.2 & 234.6 & 249.0 & 250.8 & 271.7 \\
\hline Nombre de communes & 3251 & 5468 & 9827 & 11402 & 3201 & 1140 & 515 & 437 \\
\hline $\begin{array}{l}\text { Pourcentage de la } \\
\text { population }\end{array}$ & $0.3 \%$ & $1.2 \%$ & $4.9 \%$ & $17.2 \%$ & $15.1 \%$ & $12.1 \%$ & $10.8 \%$ & $38.2 \%$ \\
\hline
\end{tabular}

Note : Les dépenses d'investissement par habitant incluent les budgets principaux et les budgets annexes, mais excluent les remboursements de la dette. Les données concernent la France métropolitaine uniquement.

Source: L'Observatoire des finances et de la gestion publique locales, https://www.collectiviteslocales.gouv.fr/ofgl. 
À court terme, un renforcement du rôle des établissements publics de coopération intercommunale dans la mise en place des infrastructures contribuerait à dégager des gains d'efficience sur les dépenses locales d'investissement. Il serait judicieux de faire en sorte que ces établissements deviennent la structure de référence pour toutes les grandes opérations d'infrastructure publique. Les investissements d'infrastructure et les dépenses d'équipement de grande envergure permettent en effet de réaliser des économies d'échelle substantielles, car les coûts unitaires diminuent si une population importante est desservie. Les dispositions asymétriques, selon lesquelles les responsabilités des municipalités sont différenciées en fonction de la taille de la population ou de critères urbains / ruraux, pourraient être davantage développées à cet égard (Allain-Dupré, 2018). La différenciation des compétences selon la catégorie d'intercommunalités va dans ce sens. Conduire des expériences pilotes, comme celles menées au Danemark dans le cadre du programme « Municipalité libre », serait également utile pour identifier les arrangements asymétriques présentant les avantages les plus importants.

À plus long terme, les fusions de communes pourraient être encouragées en faisant la preuve qu'il est possible d'améliorer les services publics, par exemple en publiant des indicateurs de performance au niveau communal (résultats des services et efficience des prestations de services, par exemple). Dans les petites communes, l'expertise est plus dispersée, ce qui a pour effet d'aggraver les problèmes de coordination. De nombreux pays de l'OCDE, comme le Japon, le Danemark et la Nouvelle-Zélande, ont procédé à des fusions de communes à grande échelle, dans le cadre de dispositifs obligatoires ou volontaires (OCDE, 2017e). Les régions pourraient également aider les communes à mieux saisir les avantages financiers d'une fusion ou bien agir en tant que médiateur, comme c'est le cas aux Pays-Bas ou en Suisse. Bien que la taille optimale des communes ne fasse pas l'objet d'un consensus, il a été démontré que les bénéfices d'une fusion seraient probablement les plus importants dans les zones urbaines où le morcellement du tissu communal a des effets négatifs sur la performance économique (Bartolini, 2015).

\section{Les transferts aux collectivités locales devraient mieux tenir compte de leurs besoins de dépenses}

Il est nécessaire de donner plus de poids aux critères de charges dans le calcul de concours financiers de l'État aux collectivités locales afin de garantir une répartition efficace des ressources. Les investissements représentent une part substantielle des dépenses des collectivités locales, en particulier des communes et des régions (Graphique 9); l'efficacité des investissements au niveau local passe par conséquent par une adéquation des ressources avec les besoins au plan local. En outre, le système de décentralisation budgétaire fait actuellement l'objet d'une refonte en profondeur, ce qui a des répercussions sur l'investissement local. La diminution des transferts de l'État aux collectivités locales à partir de 2014 a amplifié les effets du cycle électoral sur l'investissement local. La suppression progressive de la taxe d'habitation risque en outre de réduire l'autonomie budgétaire des collectivités. Il est prévu de compenser la suppression de la taxe d'habitation par le redéploiement d'autres impositions directes locales et par l'affectation de fractions d'impôt national aux collectivités territoriales (voire marginalement par des dotations de l'Etat). Cependant la réforme risque d'affaiblir quelque peu la responsabilité des collectivités, qui est de nature à favoriser la croissance et les investissements publics (Kim et Dougherty, 2018). 


\section{Graphique 9. Dépenses d'investissement des collectivités locales en pourcentage du total des dépenses}

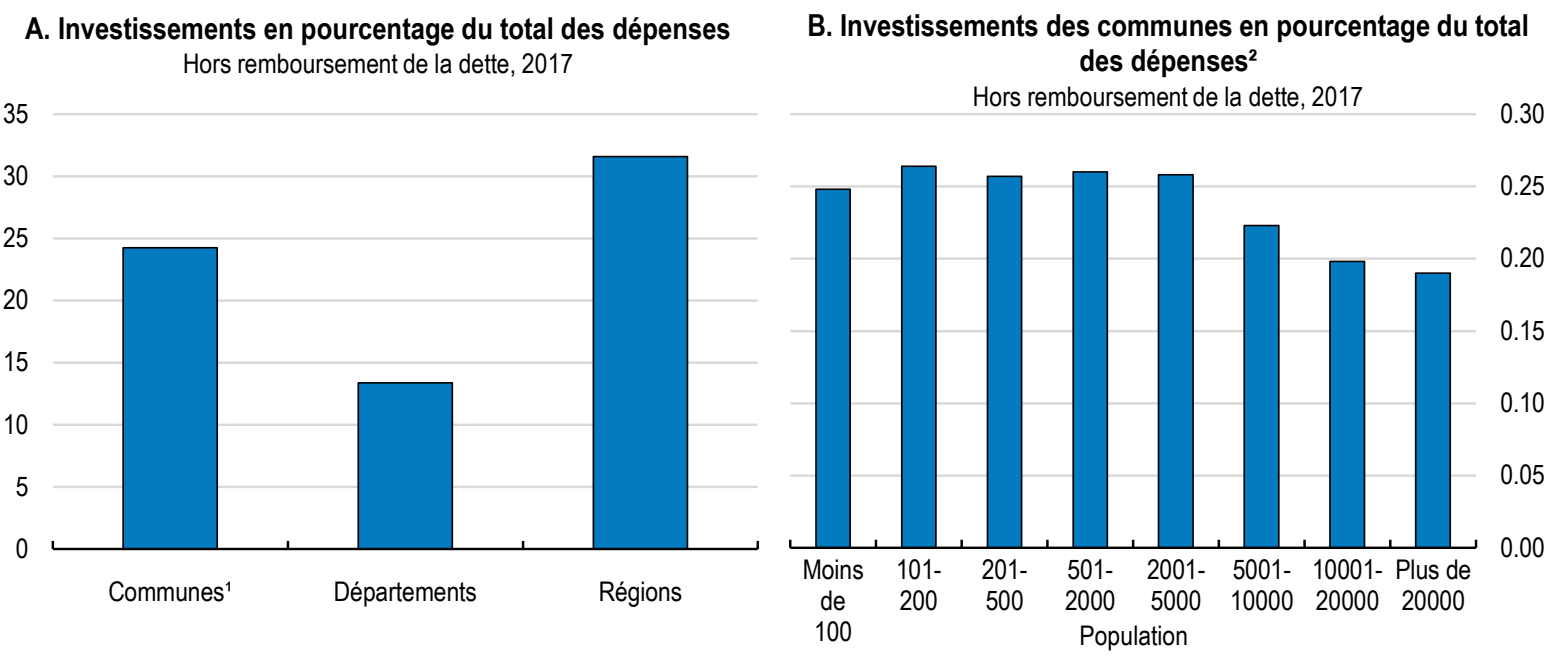

1. Les communes comprennent également les établissements publics de coopération intercommunale.

2. Données corrigées en fonction de la taille des communes.

Source : DGCL (2018), Les chiffres-clés des collectivités locales, Direction Générale des Collectivités Locales,

Paris; L'Observatoire des finances et de la gestion publique locales, https://www.collectivites-

locales.gouv.fr/ofgl.

StatLink ज्ञाs https://doi.org/10.1787/888933952803

Il serait souhaitable que le montant des transferts aux collectivités locales soit déterminé davantage en fonction de coûts unitaires standards, et moins sur les dépenses passées, afin de mieux prendre en compte leurs besoins réels de dépenses. La dotation globale de fonctionnement (DGF), la principale dotation de l'État aux collectivités locales, est répartie entre les communes, les intercommunalités ainsi que les départements. La DGF se compose d'une part forfaitaire et, selon l'échelon administratif, d'une ou de plusieurs parts de péréquation, qui sont fonction d'un grand nombre de critères. Les communes sont les principales bénéficiaires des transferts de l'État, mais ces transferts sont en partie calculés sur la base des recettes fiscales antérieures, de valeurs cadastrales obsolètes utilisées aux fins du prélèvement de l'impôt foncier et d'autres facteurs historiques, qui tendent à figer des schémas de dépenses publiques qui ne sont plus nécessairement justifiés et peuvent constituer une source importante d'inégalités entre des collectivités similaires (Cour des comptes, 2016a). À cet égard, il serait préférable que le calcul de la part forfaitaire des transferts au bloc communal repose davantage sur une méthode des coûts standards de manière à évaluer les besoins réels de dépenses des collectivités locales pour un ensemble de biens et de services collectifs de base. Dans la zone OCDE, l'Italie constitue un exemple récent de pays qui adopte progressivement une approche fondée sur les coûts standards pour diminuer le rôle des dépenses passées dans les transferts attribués aux communes (Brosio, 2018 ; Marchionni et al., 2017).

Le mécanisme de péréquation entre les communes doit être réformé afin de mieux tenir compte des disparités en matière de potentiel fiscal et de coûts de mise à disposition des biens et services publics. La part des dotations de péréquation verticale perçues par les collectivités locales a augmenté ces dernières années, ce qui est une bonne chose, même si cette hausse est due en grande partie à une diminution des concours forfaitaires. Pourtant, 
le système des dotations de péréquation est compliqué. On dénombrait ainsi 15 fonds de péréquation en 2017, pour tous les échelons administratifs : des fonds de péréquation verticale correspondant aux transferts de l'État aux collectivités locales, et des fonds de péréquation horizontale, correspondant aux transferts au sein d'un niveau d'administration locale donné. De plus, en 2018, 97, $5 \%$ des communes ont reçu au moins une dotation au titre de la péréquation verticale, ce qui suggère qu'il est possible de mieux cibler les dotations de péréquation en leur attribuant des objectifs bien définis. La péréquation horizontale pourrait encore être augmentée dans la mesure où elle représente une part relativement faible de la péréquation (environ $25 \%$ en 2018), alors même que les transferts horizontaux tendent à être plus efficaces que la péréquation verticale en termes de redistribution obtenue par unité monétaire dépensée (OCDE, 2013).

Le soutien direct en faveur de l'investissement local se fait essentiellement par le biais d'une compensation au titre du remboursement de la TVA. Le fonds de compensation pour la taxe sur la valeur ajoutée (FCTVA) est destiné à assurer aux collectivités locales une compensation de la charge de TVA qu'elles supportent sur leurs dépenses d'investissement et d'entretien des bâtiments publics et de la voirie, et qu'elles ne peuvent pas récupérer par la voie fiscale, à l'instar de ce qui se pratique dans d'autres pays de l'UE. Ce fonds représente environ les deux tiers de l'aide de l'État en matière d'investissement au plan local. Le mécanisme de remboursement du FCTVA était compliqué, en grande partie parce que les opérations de remboursement devaient être effectuées principalement manuellement, ce qui était source de complexité excessive pour l'État et les collectivités locales. On peut donc se féliciter du basculement vers une gestion automatisée du FCTVA en 2020. Cette réforme permettra d'asseoir le FCTVA sur une assiette de comptes éligibles et pourrait ainsi orienter les investissements locaux vers des secteurs spécifiques qui bénéficieront du remboursement de la TVA.

\section{Améliorer la commande publique}

La fragmentation administrative territoriale est un obstacle à des modes de gestion plus professionnels des marchés publics, ce qui peut peser sur l'efficacité des investissements publics. Les marchés publics sont estimés à $15 \%$ du PIB, les administrations locales en représentant environ la moitié. Les efforts récents pour améliorer la commande publique consistent notamment à rendre obligatoire la dématérialisation des marchés publics pour tous les niveaux administratifs en 2018. En outre, les données relatives aux marchés publics passés ont également été rendues publiques en 2018 pour favoriser la prévention et la lutte contre la corruption et promouvoir la concurrence.

Renforcer la centralisation des activités d'achat de biens et services standard achetés par le secteur public permettrait de réaliser des économies d'échelle et d'améliorer l'intégrité. Promouvoir la professionnalisation des services de marchés publics est également essentiel pour améliorer l'exécution et la maintenance des infrastructures, tout en favorisant l'intégrité des achats publics, car les marchés publics sont l'une des activités gouvernementales les plus exposées à la corruption (OCDE, 2016a). Des plates-formes électroniques de publicité et de candidature coexistent actuellement à différents niveaux administratifs (des municipalités aux régions), ce qui entraîne des coûts pour les entreprises candidates, en particulier pour les PME qui doivent adapter leurs offres à différentes platesformes en ligne. L'Union des groupements d'achats publics (UGAP), la principale centrale d'achats publics en France, est relativement modeste (environ 3 milliards d'euros en 2017 ou moins de $4 \%$ des achats publics) par rapport à d'autres pays de l'OCDE comme la Corée où la centrale d'achat concentre environ un tiers des achats du secteur public (Saussier et 
Tirole, 2015). Les régions pourraient aider à coordonner les solutions d'achat des échelons administratifs inférieurs, y compris les communes.

Il est possible d'améliorer la gouvernance des entreprises publiques locales, notamment en clarifiant leur champ d'activités et en renforçant leurs mécanismes de contrôle. Environ 1250 entreprises publiques locales (généralement sous la forme d'une société d'économie mixte) opèrent dans un large éventail de secteurs, notamment le tourisme, le logement, l'urbanisme et les industries de réseau telles que la distribution d'eau. Leur structure d'actionnaires est souvent fragmentée entre différents niveaux de collectivités locales et leur champ d'activité est souvent vaste, ce qui peut entraîner des conflits d'intérêts et nuire à la concurrence (Cour des comptes, 2017a). Une plus grande transparence sur la rémunération des membres des conseils d'administration des entreprises publiques locales augmenterait leur responsabilité, tandis que le renforcement des mécanismes de contrôle externe serait important pour préserver leur intégrité et assurer une affectation efficace de leurs ressources.

\section{Améliorer la maintenance et verdir les investissements dans l'énergie et les transports}

La France dispose d'un important stock de capital dans les secteurs de l'énergie et des transports. Cependant, les besoins en maintenance du réseau routier sont élevés, et les priorités d'investissement dans le réseau ferroviaire ont été fortement orientées vers le réseau à grande vitesse, ce qui pèse sur l'efficacité des infrastructures ferroviaires existantes. Dans le domaine de l'énergie, ce sont les centrales nucléaires vieillissantes qui posent les problèmes de maintenance les plus épineux. Il est donc prioritaire d'investir dans la maintenance des infrastructures de ces secteurs. Dans le même temps, il s'agit également de concentrer les nouveaux investissements vers la réalisation des objectifs environnementaux.

\section{Améliorer les investissements dans les infrastructures de transport}

Le total des dépenses d'investissement dans les transports, en ralentissement depuis 2013, se situe désormais au-dessous de la moyenne de l'OCDE (Graphique 10). Cette tendance est en partie la conséquence de la maturité des réseaux de transport, des contraintes budgétaires qui ont conduit le pays à revoir ses priorités dans le domaine, et du cycle électoral local. Comme dans la plupart des autres pays de l'OCDE, la majorité des investissements dans les infrastructures de transport sont destinés au réseau routier (partie B). L'analyse ci-après se concentre sur la route et le rail, destinataires de quelque $90 \%$ des investissements dans les infrastructures de transport intérieur. 
Graphique 10. Investissements dans les infrastructures de transport
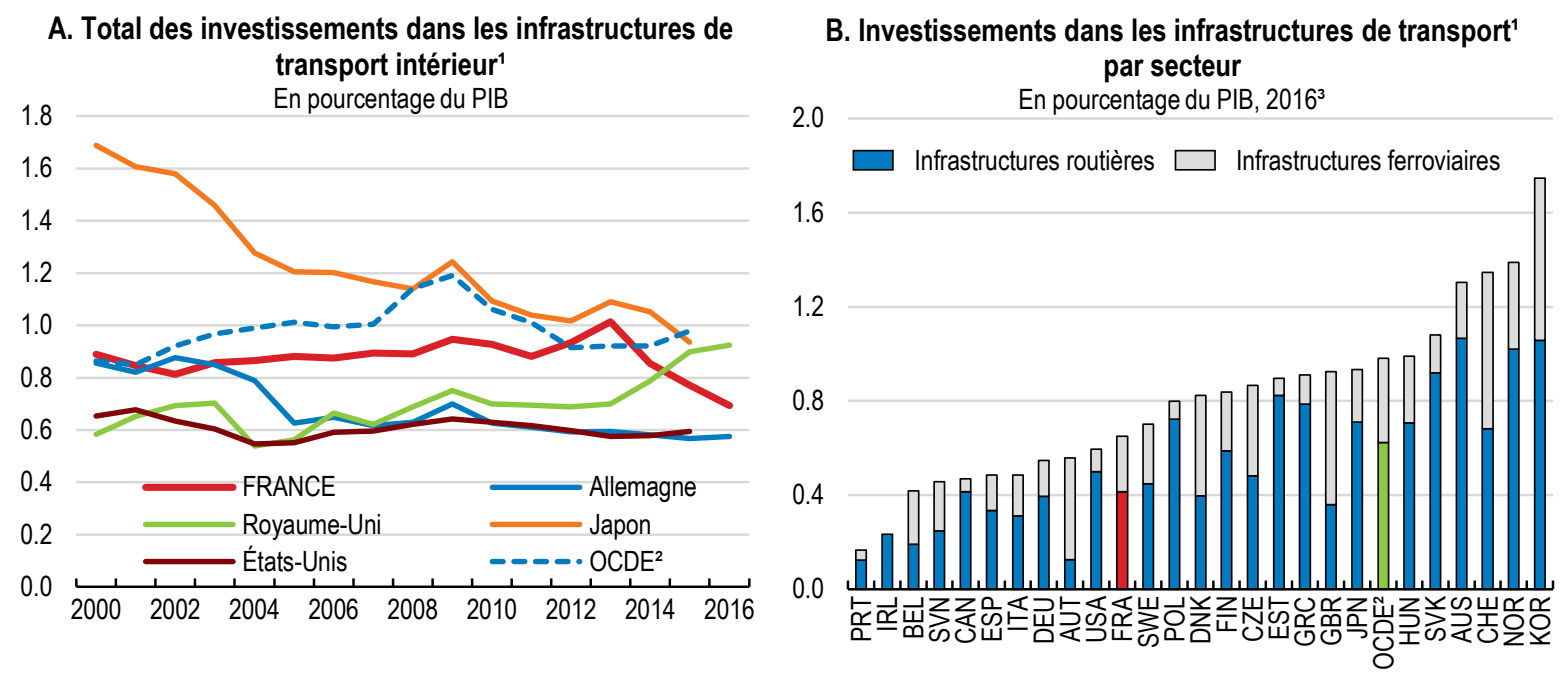

C. Densité du réseau routier Km pour $100 \mathrm{~km} 2,2016^{3}$
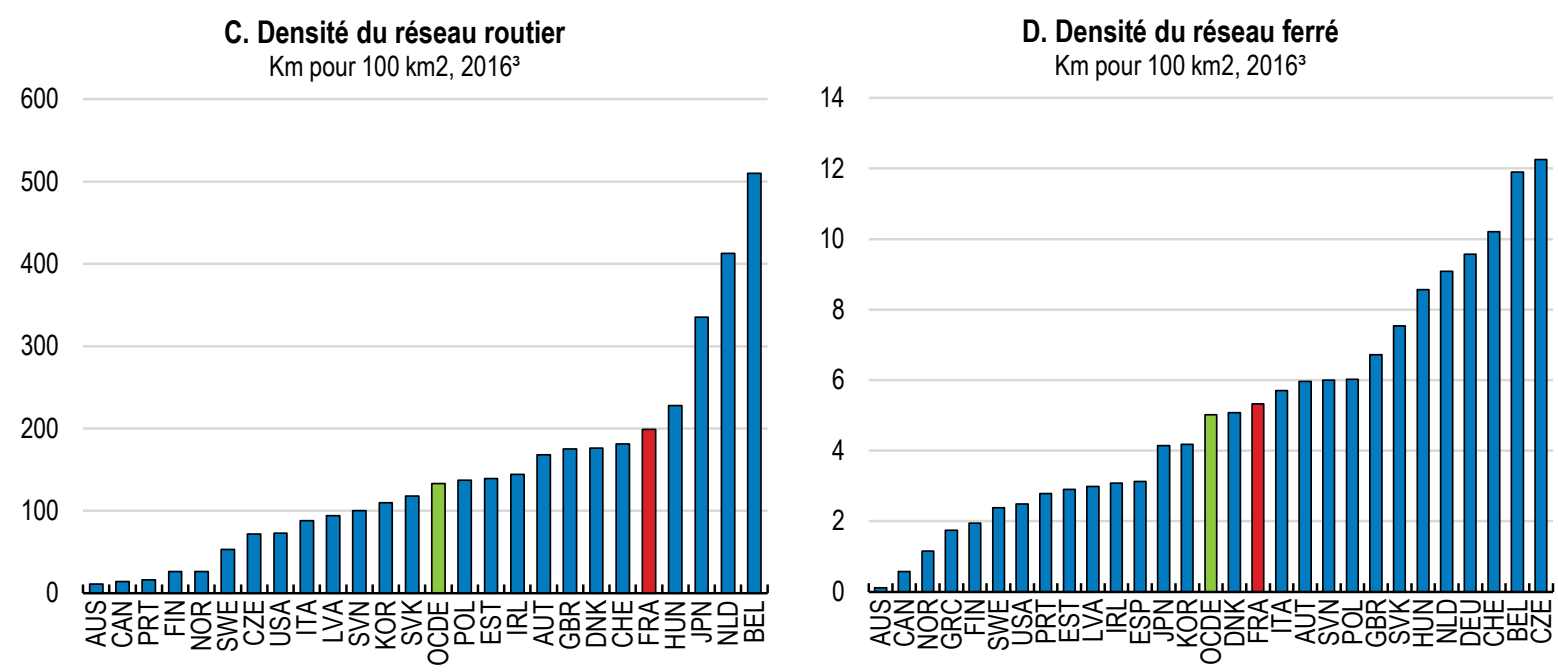

1. Les investissements dans les infrastructures de transport comprennent les dépenses publiques et les dépenses privées.

2. Moyenne non pondérée excluant le Chili, l'Irlande, les Pays-Bas et le Portugal, en raison de données limitées ou non disponibles, pour la partie A. Moyenne non pondérée des 30 pays membres ayant fourni des données pour les deux séries, pour la partie B.

3 . Ou dernière année connue.

Source: OCDE (2018), Statistiques du Forum international des transports (base de données).

StatLink ज्ञात https://doi.org/10.1787/888933952822

$\mathrm{Si}$, en France, la densité du réseau ferroviaire est proche de la moyenne de l'OCDE, celle du réseau routier est supérieure à la moyenne de l'OCDE (Graphique 10, Parties C and D), ce qui tient dans une certaine mesure à la densité de population. La planification des infrastructures de transport stratégiques obéit de plus en plus souvent à une approche multimodale des infrastructures de transport, qui tend notamment à mieux tenir compte des externalités environnementales des modes de transport (Encadré 4).

La France évalue et sélectionne ses grands projets d'infrastructure de transport selon une approche globalement cohérente avec les bonnes pratiques, mais elle aurait intérêt à prêter 
davantage attention aux interdépendances entre modes de transport, à recourir à des analyses fondées sur des scénarios, et à surveiller de plus près les coûts d'exploitation et de maintenance. Les outils de modélisation que le ministère des Transports a mis au point pour établir des prévisions des flux de trafic - indispensables pour recenser les besoins d'infrastructures de transport - et qui sont utilisés dans les analyses coûts-bénéfices ex ante, conviennent bien aux projets routiers de longue distance, mais moins aux projets routiers urbains ou multimodaux, ce qui laisse entendre qu'il est nécessaire de mieux adapter les outils de modélisation au type de projet (CEREMA, 2018).

\section{Encadré 4. Répartition des responsabilités et planification des transports}

L'État est chargé de la construction et de l'entretien des autoroutes et des routes nationales, mais il a concédé la plupart des autoroutes à des sociétés privées qui, à ce titre, en assurent la maintenance et exploitent les gares de péage. Les départements sont responsables des routes départementales et d'une partie du reste du réseau routier, tandis que les voies communales sont placées sous la supervision de la commune ou de l'intercommunalité concernée. Enfin, les investissements dans les infrastructures ferroviaires sont du ressort de l'entreprise ferroviaire publique française, la Société nationale des chemins de fer français (SNCF).

Formulé pour la première fois en 2011, le Schéma national d'infrastructures de transport (SNIT), qui a remplacé les schémas directeurs sectoriels, se veut un moyen de réfléchir selon une approche multimodale à la planification de l'ensemble des infrastructures de transport. Sa révision en 2013 puis en 2018 a permis le recensement de projets prioritaires raisonnables d'un point de vue budgétaire (Commission Mobilité 21 en 2013 et Conseil d'orientation des infrastructures en 2018) ; un projet de révision de la loi d'orientation des transports intérieurs (LOTI) de 1982 est aujourd'hui en préparation (loi d'orientation des mobilités, LOM). Tous ces plans stratégiques d'aménagement du territoire visent à fixer des objectifs à long terme, mais ne constituent pas des programmes d'investissement, ceuxci étant décidés dans des contrats de plan; de plus, les schémas directeurs ne sont pas exhaustifs, puisqu'ils n'incluent pas des projets à grande échelle tels que le Grand Paris Express (construction en région parisienne de nouvelles lignes de métro d'un coût total estimé de 35 milliards d'euros, soit $1.6 \%$ du PIB de 2017, avec un impact budgétaire sur les finances publiques) ou la planification des infrastructures aéroportuaires.

De plus, mieux documenter les hypothèses qui sous-tendent l'estimation des flux de trafic futurs dans les analyses ex ante et utiliser systématiquement des outils de modélisation aideraient à identifier les sources des erreurs de prévision et à formuler de meilleures analyses prospectives, ce qui, in fine, améliorerait la qualité des évaluations. En intégrant des approches fondées sur des scénarios au processus d'évaluation des projets, il serait également possible de mieux caractériser les incertitudes et les risques pour les décideurs, en particulier s'agissant des coûts de construction et de maintenance, qui ont souvent tendance à dépasser les montants prévus, dans le domaine du transport ferroviaire comme dans celui du transport routier (CEREMA, 2018 ; Crozet, 2013).

\section{Les transports non carbonés devraient être mieux inclus dans les plans d'investissement}

Les projets d'infrastructures de transport à long terme ne tiennent pas encore pleinement compte des transports non carbonés, malgré leur prise en compte dans les différents volets 
du PIA et dans le GPI. Pourtant, les scénarios de déploiement technologique élaborés par l'Agence internationale de l'énergie à la suite de l'accord de Paris sur le climat conclu en 2015 laissent entendre que, dans toutes les grandes économies du monde, la quasitotalité des deux roues en circulation devront être électriques au milieu des années 2040, et près de $90 \%$ de toutes les voitures devront l'être en 2060 (Agence internationale de l'énergie, 2017). Les pays à revenu élevé comme la France doivent atteindre plus rapidement la neutralité carbone - c'est-à-dire un flux de "zéro émissions nettes 》- à l'échelle de leur territoire - et en 2017, la France s'est engagée à atteindre la neutralité carbone d'ici 2050 -, ce qui suppose un déploiement anticipé des infrastructures requises, que ce soit pour l'électricité, l'hydrogène ou les carburants de synthèse (Climate Action Tracker, 2018). Il serait donc pertinent que les plans relatifs aux infrastructures de transport incluent des scénarios qui tiennent compte de la réalisation de cet objectif. Comme prévu dans le projet de loi d'orientation des mobilités, les investissements locaux doivent eux aussi soutenir une transition vers les transports publics, le vélo et la marche à pied. À noter que le cadre pour l'évaluation socio-économique des grands projets permet d'intégrer la nouvelle valeur tutélaire du carbone réestimée en 2019 dans le cadre de l'objectif de neutralité carbone en 2050.

\section{L'accent mis sur les dépenses de maintenance est le bienvenu}

La France doit consacrer davantage d'efforts à l'évaluation de l'état de son réseau routier et élaborer une stratégie de diffusion des bonnes pratiques d'entretien de ce réseau. Son réseau routier, très étendu, est principalement géré par les collectivités locales (Tableau 4). Depuis 2016, une agence est chargée d'en surveiller systématiquement la qualité (ONR, Observatoire national de la route). Le premier rapport de l'ONR paru en 2017 a montré qu'il faut un travail accru de collecte de données sur la qualité du réseau de transport routier - en particulier à l'échelle des collectivités locales - et qu'il faut améliorer les analyses de données qui sous-tendent les dépenses de maintenance. Le partage des bonnes pratiques d'entretien du réseau routier, par exemple la budgétisation pluriannuelle des dépenses ou des politiques de mutualisation des ressources des collectivités locales, pourrait générer des économies substantielles (Conseil général de l'environnement et du développement durable, 2017). Pour ce qui est du réseau routier à la charge de l'État, les dépenses de maintenance semblent limitées au regard de celles d'autres pays européens comme les Pays-Bas ou le Royaume-Uni ; et ces dépenses semblent également modestes pour maintenir le niveau de qualité des infrastructures (Ministère de la Transition écologique et solidaire, 2018). À noter le renforcement dans le cadre du GPI des dépenses de rénovation des routes, des chemins de fer, des bâtiments ainsi que des actions prévues pour la mobilité douce.

Tableau 4. Le réseau routier français en quelques chiffres

\begin{tabular}{ccl}
\hline Réseau routier, nombre total & 1064 & \multicolumn{1}{c}{ Gestion de la maintenance } \\
de km & 862 & \multicolumn{1}{c}{} \\
\hline dont autoroutes concédées & 9048 & Secteur privé \\
autoroutes non concédées & 2601 & État \\
routes nationales & 8898 & État \\
routes départementales & 377 & Départements \\
& 965 & \\
routes communales & 666 & Communes ou intercommunalités \\
\hline
\end{tabular}

Source : Observatoire national de la route, https://www.idrrim.com/ONR/. 
Les investissements ferroviaires ont été largement concentrés sur le déploiement du réseau à grande vitesse, au détriment des opérations de maintenance, ce qui semble avoir eu des effets négatifs sur la qualité perçue du réseau ferroviaire (Graphique 11). Les priorités d'investissement ont été établies en fonction des rendements économiques des différents projets, et un schéma directeur sectoriel de planification des futures lignes de train à grande vitesse a été adopté en 1992. Cependant, l'expansion du réseau à grande vitesse fait aujourd'hui l'objet d'une surveillance accrue, les lignes les plus récentes étant moins rentables et leur financement plus difficile à trouver étant donné les contraintes budgétaires avec lesquelles doivent composer les pouvoirs publics (Cour des comptes, 2014).

\section{Graphique 11. Dépenses d'investissement ferroviaire}
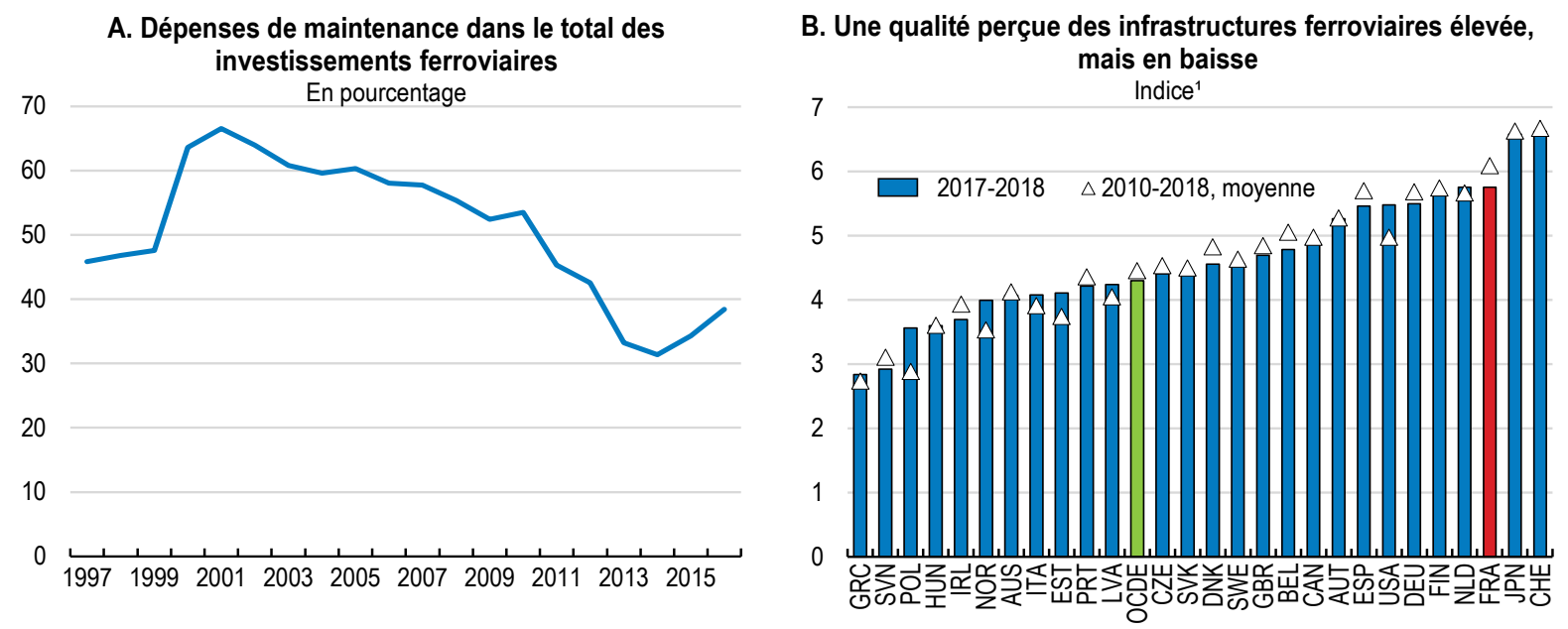

1. Indice de qualité perçue, du niveau le plus bas (0) au niveau le plus haut (7).

Source: FIT (2018), Statistiques sur les infrastructures de transport (base de données), Forum international des transports, Paris ; FEM (2018), The Global Competitiveness Report 2017-2018, Forum économique mondial, Genève.

StatLink तiाs https://doi.org/10.1787/888933952841

La loi d'orientation des mobilités devrait avoir pour effet bienvenu d'aider à rediriger les investissements ferroviaires vers les opérations de maintenance et de faciliter le trafic sur les lignes saturées aux abords de certaines grandes villes (nœuds ferroviaires). Cependant, les efforts mobilisés doivent aussi viser à améliorer les conditions cadre de la planification des investissements dans la maintenance ferroviaire. À cet égard, l'entreprise publique responsable de l'infrastructure ferroviaire (SNCF Réseau) doit améliorer la façon dont elle rend compte de son activité à l'État, de sorte que les investissements de maintenance parviennent là où ils sont le plus nécessaires, une évolution qui nécessitera sans doute des audits externes (ARAFER, 2018).

\section{Promouvoir le covoiturage peut aider à réduire les besoins d'investissement dans les transports publics}

Améliorer les services de covoiturage proposés sur plateforme numérique serait un moyen de réduire les kilométrages parcourus, les émissions et la congestion routière, sous réserve qu'ils se substituent à l'utilisation de la voiture individuelle dans les zones métropolitaines. Ils permettraient de libérer des espaces urbains coûteux actuellement occupés par des places de stationnement, et fourniraient un transport public de bonne qualité à faible coût. Dans ses analyses de la mobilité partagée, le Forum International des Transports suggère en outre 
que le covoiturage favoriserait l'accès à l'emploi et aux services publics qu'il rendrait plus faciles et équitables, notamment en zone suburbaine, et qu'il pourrait aussi être un moyen de desservir les réseaux de transports publics de grande capacité comme le métro ou le train, parfois sous-utilisés quand les usagers ont du mal à se rendre jusqu'aux stations ou aux gares de manière efficiente (Forum International des Transports, 2017).

À cet égard, il serait pertinent d'inclure des solutions de mobilité partagée dans les programmes d'aménagement du territoire comme envisagé par le projet de loi LOM. Les autorités de régulation des transports locaux pourraient faciliter la mise en place d'une plateforme unique de mise en relation des demandes de déplacement avec les offres des différents opérateurs de mobilité, laquelle plateforme pourrait faire l'objet d'une concession réglementée assortie d'une échéance, accordée à une entreprise privée à l'issue d'un processus de mise en concurrence. Il serait également judicieux d'examiner les obstacles réglementaires aux nouveaux services de transport urbain tels que les services de location de véhicule avec chauffeur, l'objectif étant de s'assurer que les exigences à l'entrée sur le marché ne sont pas trop restrictives, que la période de mise en œuvre des nouvelles mesures réglementaires est suffisante, et que les examens organisés par les associations professionnelles (Chambres des métiers et de l'artisanat) sont équitables dans tous les départements (Autorité de la concurrence, 2017).

Les services de covoiturage à la demande proposés sur plateforme numérique sont également un moyen d'améliorer l'offre de transport en zone rurale, même quand la densité de population est faible. La Norvège a obtenu des résultats prometteurs en proposant des services de bus qui, au lieu d'emprunter un trajet fixe à une heure fixe, acheminent les passagers de leur point de départ à leur point d'arrivée (service de porte à porte) à l'heure de leur choix (Dotterud et Skollerud, 2015). En offrant ainsi, spécialement aux jeunes et aux seniors, de meilleures possibilités d'accès aux activités sociales et culturelles et aux services de santé, ces solutions nouvelles contribuent à améliorer le bien-être.

\section{Développer la tarification efficace de l'usage des infrastructures routières aiderait à répondre aux besoins de maintenance et à promouvoir d'autres modes de transport}

La France gagnerait à promouvoir la tarification efficace de l'usage des infrastructures routières. En octobre 2014, elle a renoncé à instaurer une taxe sur les poids lourds (dite écotaxe) compte tenu des dissensions suscitées par la tarification routière. Pourtant, les poids lourds sont généralement la cause d'une grande partie des travaux d'entretien des infrastructures routières, et ils contribuent grandement à augmenter la pollution de l'air. Un certain nombre de pays de l'OCDE comme l'Allemagne, la Belgique ou la Slovaquie taxent l'utilisation des routes par les camions en leur appliquant une tarification kilométrique. À l'avenir, l'adhésion du public à de tels mécanismes de tarification pourrait être encouragée par de plus larges campagnes de sensibilisation expliquant les besoins d'entretien du réseau routier, les avantages d'une circulation plus fluide et les effets néfastes qu'une pollution atmosphérique urbaine trop élevée peut avoir sur la santé.

De plus, promouvoir la solution du péage urbain, qui consiste à imposer aux automobilistes le paiement d'une redevance à l'entrée d'une zone urbaine spécifique, aiderait à lutter contre la congestion et financer les transports publics. Le projet de loi LOM prévoyait de faciliter la mise en place de péages urbains, mais cette mesure a été abandonnée à la suite des manifestations des gilets jaunes. Des villes européennes telles que Londres, Stockholm ou Milan ont mis en place des mécanismes de péage urbain et obtenu des résultats positifs (Eliasson, Hultkrantz et Smidfelt Rosqvist, 2009). À cet égard, il serait pertinent, en France, 
de lever la restriction qui n'autorise les autorités locales à expérimenter des systèmes de péage urbain que pendant une durée de trois ans, étant donné le temps qui leur est nécessaire pour atteindre le seuil de rentabilité de tels investissements. Des mesures d'accompagnement à destination des ménages modestes, à l'image des mesures prises à Rome ou Stockholm, seront également nécessaires pour encourager l'adhésion du public à un tel dispositif.

Le transport ferroviaire de marchandises, une alternative faiblement carbonée au fret routier, affiche une tendance baissière même si sa part de marché est stable depuis quelques années (Graphique 12). L'Autriche et la Suisse sont deux pays de l'OCDE ayant réussi à développer substantiellement le marché du fret ferroviaire, sans conditions préalables favorables à ce mode de transport, telles que la présence d'industries lourdes ou la production de matières premières. Pour obtenir ces résultats, l'Autriche et la Suisse ont appliqué un ensemble de mesures de réglementation - par exemple, une taxe sur les poids lourds, l'application de restrictions de dimension et de poids aux camions, l'interdiction faite aux poids lourds de se déplacer la nuit et le week-end- et de politiques d'investissement dans la rénovation ou la construction de lignes ferroviaires afin d'améliorer les performances du fret ferroviaire (Cour des comptes de l'Union européenne, 2016).

Améliorer les performances des services de fret ferroviaire et faire en sorte que les autorités assurent un soutien cohérent sont deux leviers d'action pour contribuer au développement du transport ferroviaire de marchandises. Par le passé, certaines politiques nationales sont allées contre l'objectif de développer le fret ferroviaire (par exemple, suppression de l'écotaxe sur les camions en 2014, et augmentation du poids total autorisé pour les camions en 2012). Il sera également important d'améliorer la qualité du service de fret ferroviaire en améliorant celle du réseau ferroviaire lui-même, puisqu'il existe une relation entre l'insuffisance de la qualité de service et l'insuffisance de la maintenance des lignes ferroviaires les plus utilisées pour le fret (Cour des comptes, 2017b).

\section{Graphique 12. Le fret ferroviaire est engagé sur la mauvaise voie}

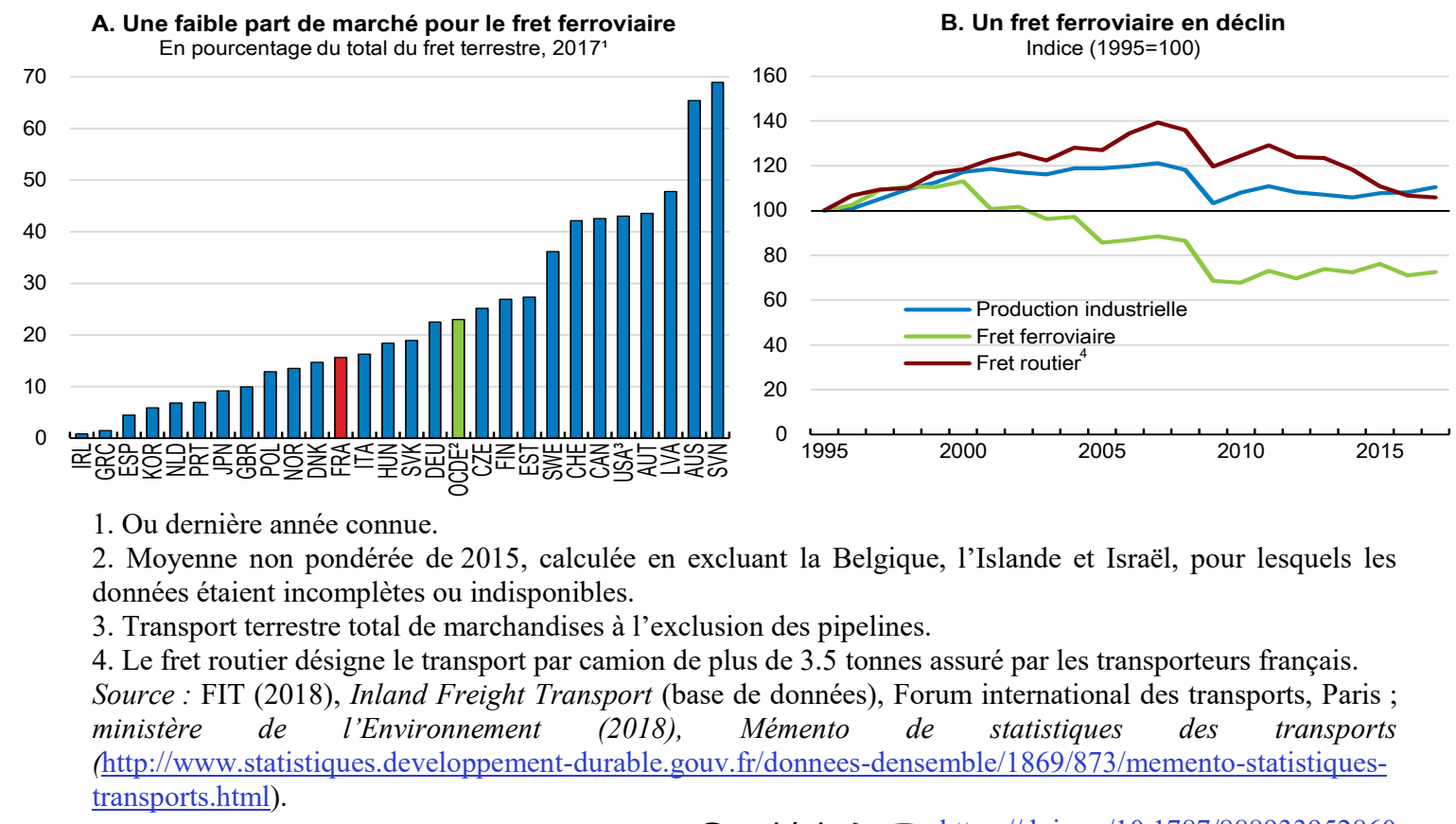

StatLink 게개 https://doi.org/10.1787/888933952860 
Les investissements devraient aussi viser à augmenter le nombre de terminaux de chargement/déchargement des trains de marchandises et à améliorer les connexions multimodales. Il semble important de moderniser les axes ferroviaires qui relient les ports maritimes à l'intérieur du territoire, en tenant compte des résultats des analyses coûtsbénéfices. Par exemple, les liaisons ferroviaires à destination des deux premiers ports maritimes français - Le Havre et Marseille - sont beaucoup plus lentes que les liaisons routières, la vitesse moyenne des trains de marchandises relevée entre Paris et Le Havre ne dépassant pas $6 \mathrm{~km} / \mathrm{h}$ (Parlement européen, 2015). Il convient à cet égard de saluer le récent développement des autoroutes ferroviaires (ferroutage), une forme particulière de fret ferroviaire consistant à charger des semi-remorques sur des wagons en toute simplicité et flexibilité - par opposition aux autres formes de fret ferroviaire qui nécessitent généralement le chargement de conteneurs par manutention verticale (grue ou portique). Continuer les efforts au service du déploiement d'autoroutes ferroviaires avec les pays voisins, prévue par les pouvoirs publics, semble également justifiée.

\section{Promouvoir des infrastructures énergétiques appropriées}

Pour se conformer à ses engagements internationaux et européens, la France a voté en 2015 une loi relative à la transition énergétique pour la croissance verte qui définit ses objectifs énergétiques et climatiques. La part des énergies renouvelables dans la consommation d'énergie finale devrait atteindre $32 \%$ d'ici 2030, en cohérence avec l'objectif de référence de l'Union européenne, tandis que les émissions de gaz à effet de serre (GES) devraient être ramenées en 2030 à un niveau inférieur de $40 \%$ à leur niveau de 1990. Dans le même temps, il est prévu que la part du nucléaire dans la production d'électricité soit limitée à $50 \%$ en 2035 (contre $72 \%$ en 2017) et la puissance nucléaire installée plafonnée à 63.2 GW dans la version révisée de la Programmation pluriannuelle de l'énergie. En 2017, le Plan Climat a permis de mettre à jour les objectifs et les instruments d'action, qui sont notamment d'atteindre la neutralité carbone à l'horizon 2050 et l'interdiction de la vente de véhicules alimentés aux carburants fossiles à compter de 2040 .

Ces objectifs environnementaux sont cependant nombreux et parfois contradictoires, étant donné les défis que doivent relever les autorités pour respecter l'objectif de réduction de la part du nucléaire à $50 \%$ du parc électrique à moyenne échéance, tout en assurant la sécurité des approvisionnements énergétiques et en maintenant aussi bas que possible l'empreinte carbone de la production d'énergie (OCDE, 2016b). La part de l'électricité d'origine renouvelable n'a que peu augmenté depuis les années 1990, même si la place importante de l'électronucléaire permet de limiter les émissions de $\mathrm{CO}_{2}$ (Graphique 13). 
Graphique 13. Peu d'énergies renouvelables dans le parc électrique, mais peu d'émissions de CO2 aussi
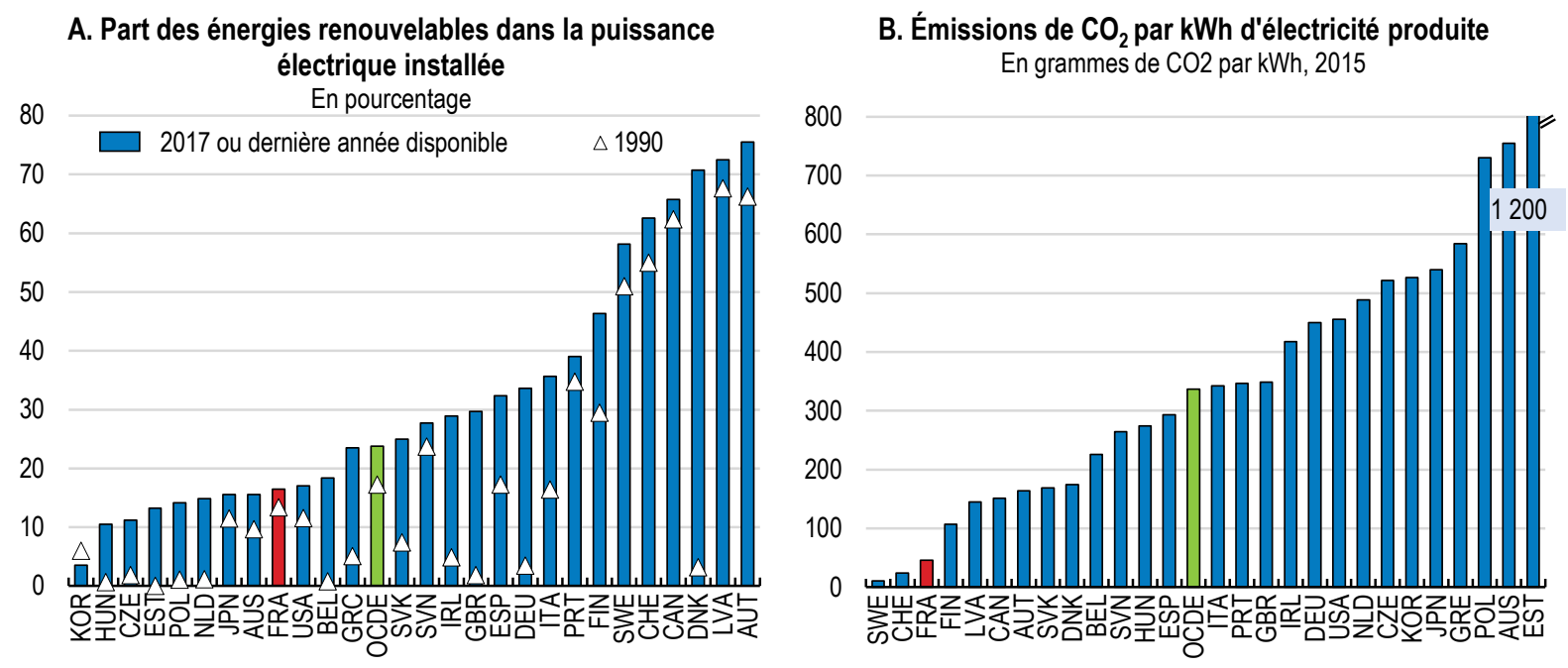

Source: OCDE (2019), Indicateurs de croissance verte (base de données); AIE (2019), « Emissions per kWh of electricity and heat output », IEA CO2 Emissions from Fuel Combustion Statistics (base de données).

StatLink :inst https://doi.org/10.1787/888933952879

\section{Augmenter l'efficacité énergétique pour limiter la demande d'énergie}

Augmenter l'efficacité énergétique des bâtiments résidentiels et publics est un volet important du Grand plan d'investissement (Encadré 2). Investir dans l'efficacité énergétique peut être bénéfique à de multiples niveaux : cela réduit la demande d'énergie et les coûts associés et peut favoriser la réalisation d'autres objectifs, par exemple l'amélioration de la qualité sanitaire des environnements intérieurs. Le chauffage résidentiel demeure l'une des utilisations finales les plus émettrices de $\mathrm{CO}_{2}$, même s'il a également contribué le plus aux économies d'énergie depuis 2000 (Graphique 14). Le Grand plan d'investissement alloue 9 milliards d'euros à l'augmentation de l'efficacité énergétique des bâtiments résidentiels et publics au cours de la période 2018-22, une enveloppe qui vient s'ajouter aux mesures existantes d'amélioration des performances énergétiques du secteur résidentiel (certificats d'économies d'énergie, réglementation thermique applicable aux bâtiments neufs, prêts subventionnés). Quelque $85 \%$ de ces 9 milliards d'euros devraient aller aux logements sociaux et aux bâtiments du secteur public, en particulier ceux des collectivités locales. Toutefois, pour tirer le maximum de bénéfices de ce financement supplémentaire, la France doit continuer de tout mettre en œuvre pour améliorer les compétences et la certification des professionnels du secteur des performances énergétiques, attendu que, par le passé, la qualité des diagnostics énergétiques et des travaux de rénovation a pu être inégale (OCDE, 2016b). Il est également important de veiller à ce que les mesures de soutien dépendent d'une amélioration globale de la performance énergétique des bâtiments. 


\section{Graphique 14. Des gains d'efficacité énergétique qui proviennent essentiellement du secteur} résidentiel

\section{A. Émissions de $\mathrm{CO}_{2}$ par type d'utilisation finale, $2015^{1}$}

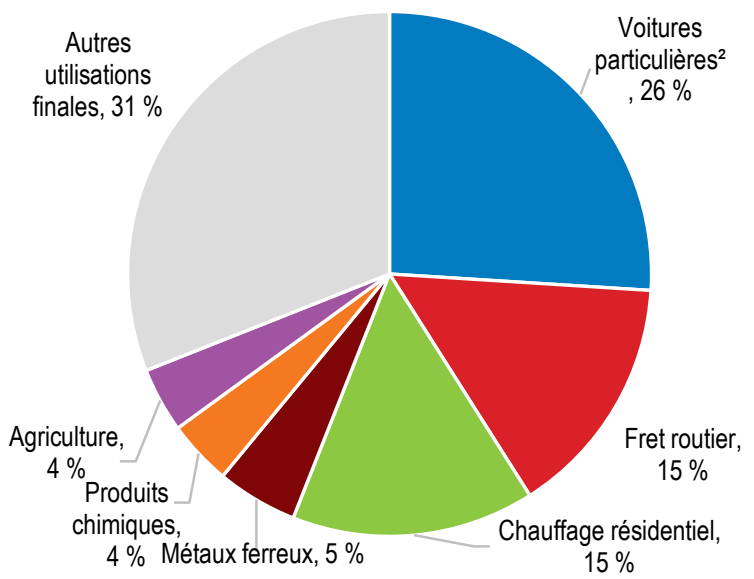

\section{B. Estimation des économies d'énergie cumulées par secteur 2000-2015, pétajoules ${ }^{3}$}

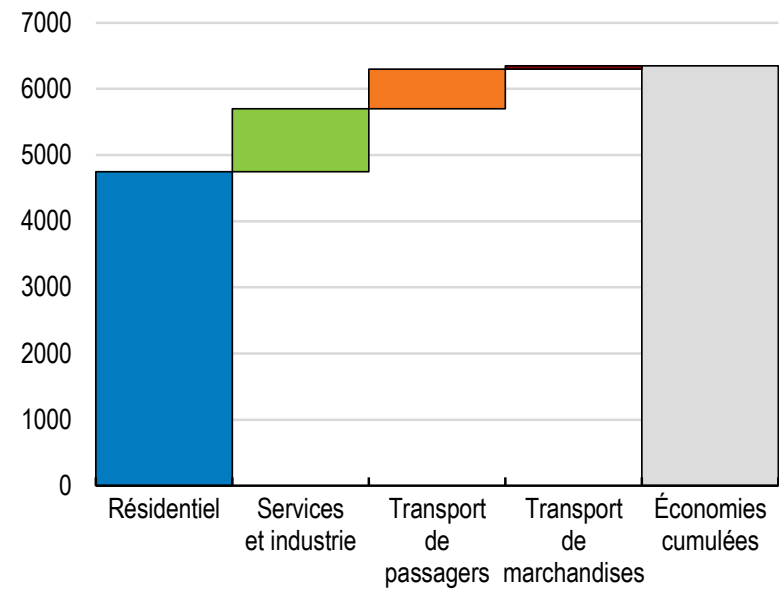

1. Y compris les émissions dues à la production d'électricité et de chaleur.

2. Les voitures particulières incluent les berlines, les véhicules tout-terrain de loisir et les véhicules utilitaires utilisés à des fins personnelles.

3. Résultats d'une ventilation de l'AIE recouvrant environ $98 \%$ de la consommation d'énergie finale en France.

Source : AIE (2017), Energy Efficiency Indicators: Highlights, Agence internationale de l'énergie, Paris.

StatLink ज्ञाs https://doi.org/10.1787/888933952898

\section{Les défis économiques de l'électronucléaire}

La rénovation des centrales nucléaires représente un coût important, dont la justification économique dépend de la durée de fonctionnement prévue pour ces installations. En France, l'énergie nucléaire est la première source d'électricité (Graphique 15). Les 58 réacteurs du pays, implantés sur 19 sites, sont tous exploités par l'énergéticien public Électricité de France (EDF) - une entreprise cotée en bourse dont l'État détient 83.7\% du capital. La plupart des réacteurs nucléaires ont été construits dans les années 1980 en l'espace de quinze ans, quand une hypothèse largement partagée était qu'ils auraient une durée d'exploitation fixée à 40 ans (Tableau 5). Toutefois, l'Autorité de sûreté nucléaire (ASN) ne fixe pas de limite maximale au nombre d'années d'exploitation. Au lieu de cela, l'ASN effectue tous les dix ans, pour chaque réacteur, un examen approfondi au cours duquel la conformité aux références de sécurité et le vieillissement des matériaux sont évalués.

Pour continuer à obtenir les prolongations de vie de 10 ans de son parc de réacteurs au-delà de la durée de vie initialement prévue de 40 ans, et afin de mettre en œuvre les améliorations de sûreté décidées après l'accident de Fukushima en 2011, EDF a lancé dès 2014 un grand programme d'investissement à long terme (le grand carénage). EDF estime le coût de ce programme, qui vise en particulier à porter la durée de vie de la plupart des réacteurs à 50 ans, dans la perspective d'une extension à 60 ans, ce qui est habituel aux États-Unis, à 48 milliards EUR sur la période 2014-2025. Ce chiffre équivaut à faire passer les investissements de maintenance des centrales nucléaires de quelque 3 milliards d'euros par an à environ 4,2 milliards d'euros par an. 


\section{Graphique 15. Une production d'électricité d'origine principalement nucléaire}

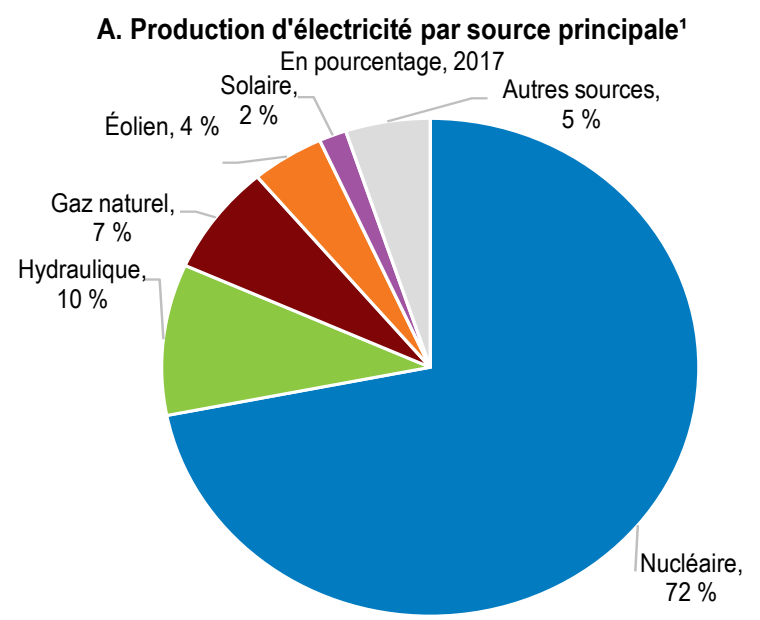

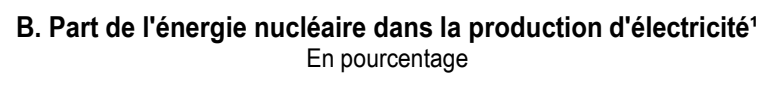

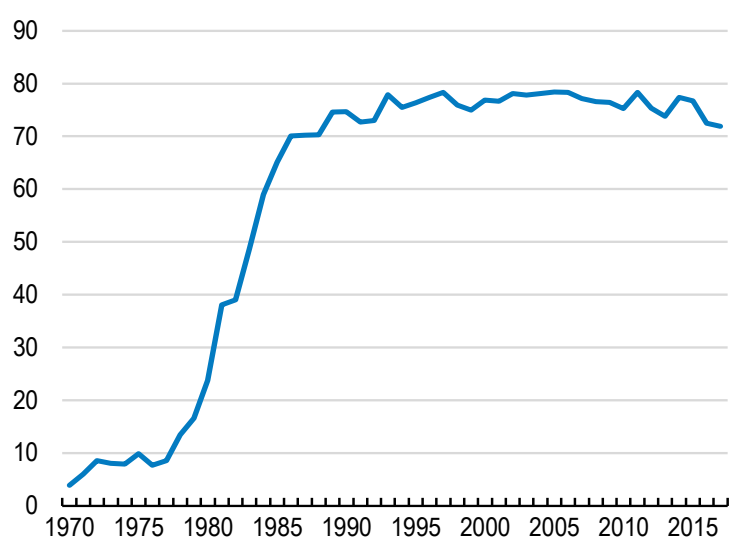

1. Production d'électricité brute.

Source: AIE (2018), Electricity Information Statistics - OECD Electricity and Heat Generation (base de données), Agence internationale de l'énergie, Paris.

StatLink त्गाजम https://doi.org/10.1787/888933952917

Tableau 5. Un parc électronucléaire vieillissant

Années de mise en service des réacteurs à eau pressurisée en France, 1977-99

\begin{tabular}{|c|c|c|c|c|c|c|c|}
\hline 1977 & Fessenheim 1 & Fessenheim 2 & & & & & \\
\hline 1978 & Bugey 2 & Bugey 3 & & & & & \\
\hline 1979 & Bugey 4 & Bugey 5 & & & & & \\
\hline 1980 & Tricastin 1 & Gravelines 1 & Tricastin 2 & Tricastin 3 & $\begin{array}{l}\text { Gravelin } \\
\text { es } 2\end{array}$ & $\begin{array}{l}\text { Dampier } \\
\text { re } 1\end{array}$ & Gravelines 3 St-Laurent B 1 \\
\hline 1981 & Dampierre 2 & $\begin{array}{l}\text { St-Laurent B } \\
2\end{array}$ & Blayais 1 & Dampierre 3 & $\begin{array}{l}\text { Tricastin } \\
4\end{array}$ & $\begin{array}{l}\text { Gravelin } \\
\text { es } 4\end{array}$ & Dampierre 4 \\
\hline 1982 & Blayais 2 & Chinon B 1 & & & & & \\
\hline 1983 & Cruas 1 & Blayais 4 & Blayais 3 & Chinon B 2 & & & \\
\hline 1984 & Cruas 3 & Paluel 1 & Cruas 2 & Paluel 2 & $\begin{array}{l}\text { Gravelin } \\
\text { es } 5\end{array}$ & Cruas 4 & \\
\hline 1985 & St-Alban 1 & Paluel 3 & $\begin{array}{l}\text { Graveline } \\
\text { s } 6\end{array}$ & $\begin{array}{l}\text { Flamanville } \\
1\end{array}$ & & & \\
\hline 1986 & Paluel 4 & St-Albans 2 & $\begin{array}{l}\text { Flamanvill } \\
\text { e } 2\end{array}$ & Chinon B 3 & $\begin{array}{l}\text { Catteno } \\
\text { m } 1\end{array}$ & & \\
\hline 1987 & Cattenom 2 & Nogent 1 & $\begin{array}{l}\text { Belleville } \\
1\end{array}$ & Chinon B 4 & & & \\
\hline 1988 & Belleville 2 & Nogent 2 & & & & & \\
\hline 1990 & Cattenom 3 & Penly 1 & Golfech 1 & & & & \\
\hline 1991 & Cattenom 4 & & & & & & \\
\hline 1992 & Penly 2 & & & & & & \\
\hline 1993 & Golfech 2 & & & & & & \\
\hline 1996 & Chooz B 1 & & & & & $900 \mathrm{MW}$ & \\
\hline 1997 & Chooz B 2 & Civaux 1 & & & & $\begin{array}{l}1300 \mathrm{M} \\
\mathrm{W}\end{array}$ & \\
\hline 1999 & Civaux 2 & & & & & $\begin{array}{l}1450 M \\
W\end{array}$ & \\
\hline
\end{tabular}

Note: Un réacteur EPR de $1630 \mathrm{MW}$ de troisième génération devrait entrer en service à Flamanville (Normandie) en 2020 ou 2021.

Source : RTE (2016), Bilan prévisionnel, Paris. https://www.rte-

france.com/sites/default/files/bp2016_complet_vf.pdf 
Le parc électronucléaire existant produit une électricité bon marché - y compris en tenant compte du coût du programme grand carénage - et ce prix se compare actuellement favorablement par rapport à l'électricité obtenue à partir d'autres sources peu carbonées (Agence internationale de l'énergie, 2016). Cependant, les coûts futurs de la production électronucléaire sont entachés de grandes incertitudes (Cour des comptes, 2016b). Les coûts de l'électronucléaire dépendent très fortement de la quantité d'électricité produite par $\mathrm{kW}$ (facteur de charge), et les incertitudes sont liées en partie à l'évolution des normes de sûreté. La construction du tout nouvel EPR (Evolutionary Power Reactor) de Flamanville a accumulé les retards et les difficultés techniques (le démarrage du réacteur est prévu en 2020 ou 2021, contre l'échéance de 2012 envisagée à l'origine). Les dépassements de coûts qui se sont multipliés pour l'EPR en Finlande ont aussi conduit à une restructuration de la filière électronucléaire française.

Il est nécessaire de maintenir une surveillance étroite et de veiller à la bonne adéquation des mécanismes de financement du démantèlement des installations nucléaires et de la gestion des déchets à long terme. L'acceptation sociale de l'électronucléaire exige que l'on s'assure de la bonne gestion du démantèlement des installations et de la gestion des déchets, et de la disponibilité des fonds nécessaires en temps voulu. À la fin de 2016, EDF estimait à 75 milliards d'euros les charges de démantèlement de l'ensemble des installations nucléaires et de gestion à long terme des déchets associés, mais il est probable qu'il s'agisse d'une sous-estimation (Assemblée Nationale, 2018). En outre, les montants provisionnés pour le démantèlement des installations et la gestion des déchets à long terme dépendent beaucoup du taux d'actualisation choisi : le gouvernement impose aujourd'hui un taux révisé pour que les chiffres soient le reflet d'un prix davantage fondé sur le marché, et pour que le taux d'actualisation français soit plus proche de celui que d'autres juridictions appliquent pour calculer leurs propres provisions (par exemple, Belgique ou Suisse). Par ailleurs, l'Autorité de sûreté nucléaire (ASN) a souligné qu'EDF ne présente pas les informations suffisantes pour que l'ASN prenne position sur la complétude des charges financières concernant le démantèlement des installations et la gestion des déchets à long terme (Autorité de sûreté nucléaire, 2017), ce qui laisse entendre que davantage d'efforts sont nécessaires pour améliorer la transparence de l'adéquation des montants provisionnés pour le démantèlement des installations et la gestion des déchets à long terme.

\section{Promouvoir les sources d'énergie renouvelables}

Le soutien aux énergies renouvelables prend la forme de tarifs de rachat, de crédits d'impôt et d'une taxe sur le carbone. Pour encourager la production d'électricité d'origine renouvelable, les pouvoirs publics ont prévu des obligations d'achat pour les installations de petite taille et des appels d'offres pour les plus grandes. Pour encourager la production de chaleur d'origine renouvelable, ils proposent des aides à l'investissement via un Fonds Chaleur ainsi que, pour les ménages, un crédit d'impôt pour la transition énergétique (CITE). Le soutien public aux énergies renouvelables bénéficie de façon disproportionnée à la production d'électricité qui, en effet, contribue relativement moins aux objectifs de réduction des émissions de $\mathrm{CO} 2$ que la production de chaleur, ce qui donne à penser qu'il faudrait augmenter les mesures en faveur de cette dernière (Cour des comptes, 2018c).

Il serait pertinent de poursuivre l'examen de l'adéquation du mécanisme de financement du Fonds Chaleur. Administré par l'Agence de l'environnement et de la maîtrise de l'énergie (ADEME), le Fonds chaleur est le principal mécanisme public d'aide à l'investissement dans la production de chaleur d'origine renouvelable. Il est destiné aux collectivités locales, aux logements collectifs et aux entreprises. Les sources renouvelables de la production de chaleur se substituent généralement à des sources fossiles, tandis que 
celles de la production d'électricité tendent à remplacer l'énergie nucléaire, déjà faiblement carbonée. Pourtant, la production de chaleur d'origine renouvelable ne reçoit que $10 \%$ des aides publiques aux énergies renouvelables, qui bénéficient de façon disproportionnée à la production d'électricité d'origine renouvelable. Qui plus est, le déploiement d'installations de production de chaleur d'origine renouvelable est entravé par la limitation des ressources du Fonds Chaleur depuis quelques années (CGEDD et CGE, 2018). C'est pourquoi, l'augmentation prévue du budget du Fonds chaleur en 2019 est bienvenue; il conviendrait aussi, à l'avenir, d'ajuster périodiquement le soutien à la production de chaleur d'origine renouvelable.

Formulées pour la première fois en 2015, la Programmation pluriannuelle de l'énergie (PPE) et la Stratégie nationale bas-carbone (SNBC) ont vocation à être régulièrement mises à jour avec toutes les parties prenantes concernées, le but étant de disposer d'un cadre intégré de planification des investissements énergétiques dans le respect des objectifs énergétiques et climatiques. L'horizon de la PPE est de 10 ans, tandis que l'horizon de la SNBC est de 15 ans ; et il est prévu de les actualiser tous les cinq ans, la prochaine révision devrait être publiée à la mi-2019. Ces deux documents sont utilisés pour orienter les aides publiques vers les renouvelables en formulant des trajectoires détaillées au service de la réalisation des objectifs environnementaux à long terme.

Il manque cependant des mécanismes formels permettant d'ajuster les mesures de soutien aux énergies renouvelables quand les indicateurs annuels de performance s'éloignent trop des trajectoires définies dans la Programmation pluriannuelle de l'énergie et la Stratégie nationale bas-carbone (OCDE, 2016b). À cet égard, il serait utile de charger une institution de conduire des examens annuels et d'élaborer des scénarios à long terme, pour pouvoir réviser régulièrement le soutien public aux mesures de lutte contre le changement climatique et de transition énergétique. Une telle institution viendrait se substituer aux différentes structures actuellement actives dans ce domaine, mais avec des synergies insuffisantes ; elle pourrait être inspirée du Committee on Climate Change du RoyaumeUni ou, en France, du Conseil d'orientation des retraites, mandaté pour conduire des analyses approfondies du régime français de retraite avec la participation et le soutien d'un large éventail de parties prenantes. Le Haut Conseil pour le Climat, institué à la fin de 2018, semble répondre à cette préoccupation, mais il est encore trop tôt pour juger de son efficacité.

\section{Développer les interconnexions électriques}

La France doit continuer d'investir dans ses interconnexions avec le réseau électrique européen afin d'assurer la sécurité de ses approvisionnements énergétiques et une meilleure intégration de la production d'électricité d'origine renouvelable. Ses importants moyens de production de base en raison de la prépondérance de l'électronucléaire lui garantissent une grande quantité d'électricité disponible ; néanmoins, elle doit disposer d'une capacité d'importation suffisante pour maintenir la sécurité de ses approvisionnements pendant les pointes de demande, même si elle est en général exportatrice nette d'électricité (Agence internationale de l'énergie, 2016). Le réseau électrique français est interconnecté avec ceux de six pays limitrophes, mais les lignes qui relient la France à l'Espagne et à l'Italie sont saturées la plupart du temps (Agence internationale de l'énergie, 2016). Selon les données de la Commission européenne La France est l'un des 11 États membres de l'UE dont la part de la capacité d'interconnexion d'import dans la puissance installée était inférieure à $10 \%$ en 2017, ce qui est conforme avec les autres grands pays européens et il est prévu qu'elle atteigne cet objectif de $10 \%$ défini par l'UE d'ici 2020 (Graphique 16; Commission européenne, 2017). Dans ce contexte, RTE - le gestionnaire du réseau de 
transport d'électricité de la France - a mis en service une nouvelle ligne d'interconnexion avec l'Espagne en 2015, et de nouvelles lignes d'interconnexion avec l'Italie et le Royaume-Uni sont en construction (RTE, 2017). La France doit toutefois accélérer ses investissements dans les interconnexions avec les pays voisins à condition que les nouvelles lignes d'interconnexion obtiennent des évaluations socio-économiques favorables.

Graphique 16. Une faible capacité d'interconnexion avec le marché européen de l'électricité

En pourcentage de la puissance électrique installée, 2016

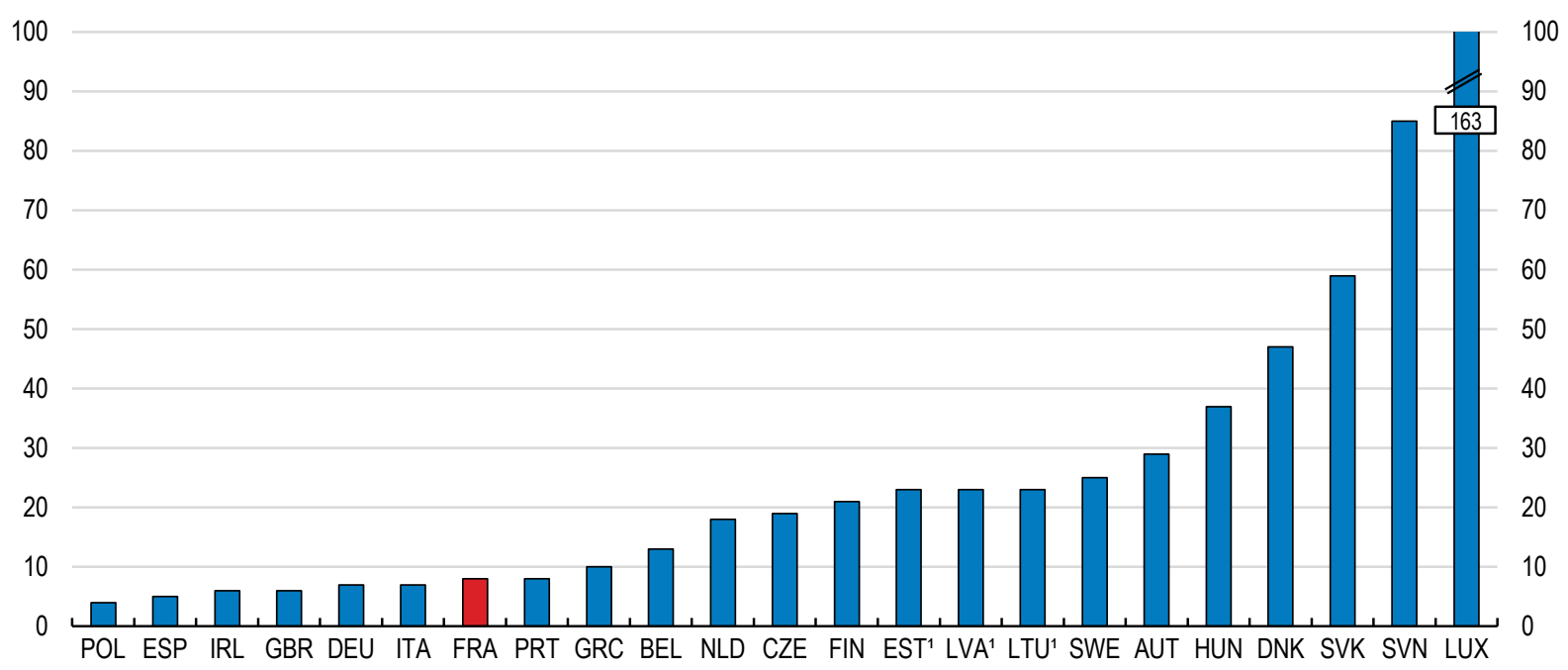

1. Le pourcentage de $23 \%$ indiqué pour l'Estonie, la Lettonie et la Lituanie se rapporte à l'interconnexion de l'ensemble de la zone baltique avec le réseau électrique européen, ces trois pays n'étant pas encore entièrement synchronisés avec le réseau européen.

Source: Commission européenne (2017), Monitoring progress towards the Energy Union objectives - key indicators, Deuxième rapport sur l'état de l'union de l'énergie, Commission européenne, Bruxelles; d'après des données d'ENTSO-E.

StatLink त्राज़ https://doi.org/10.1787/888933952936

\section{Favoriser la transformation numérique et l'innovation}

\section{Favoriser une transition plus rapide vers le numérique}

\section{La qualité des infrastructures numériques est modeste}

La qualité des infrastructures numériques, mesurée par le taux de pénétration du haut débit, sa vitesse et son prix, est en deçà de celle des pays les mieux classés à cet égard (Graphique 17). Or, les réseaux à très haut débit sont essentiels pour récolter pleinement les fruits de la révolution numérique qui transforme les interactions entre les individus, les entreprises et les pouvoirs publics. Cela passe par des infrastructures matérielles satisfaisantes, notamment leurs déclinaisons les plus avancées, qui sont nécessaires aux services fondés sur des volumes importants de données.

Comme d'autres pays de l'OCDE, la France s'est fixé en 2013 des objectifs nationaux en matière de haut débit, suivant lesquels tous les ménages, les entreprises et les administrations doivent avoir accès à un réseau à très haut débit - c'est-à-dire au moins égal à $30 \mathrm{Mbit} / \mathrm{s}$ - d'ici à 2022 (Plan France Très Haut Débit). Le financement des 
investissements réalisés est assuré intégralement par des entreprises privées dans les zones urbaines, tandis qu'en zone rurale, il est réparti entre les secteurs public et privé. Les investissements nécessaires pour atteindre les objectifs définis - aussi bien en zone rurale qu'en zone urbaine - représentent 20 milliards d'euros, dont les deux tiers seront financés par le secteur privé et le reste par l'État et les collectivités locales ainsi que par les fonds structurels de l'UE. Néanmoins, les objectifs figurant dans le Plan France Très Haut Débit sont loin de ceux retenus par les pays de l'OCDE les plus performants dans ce domaine, tels que la Corée et le Japon, ou des objectifs de connectivité fixés par l'UE à l'horizon 2025 (OCDE, 2018e).

\section{Graphique 17. Il est possible d'améliorer la qualité des infrastructures à haut débit}
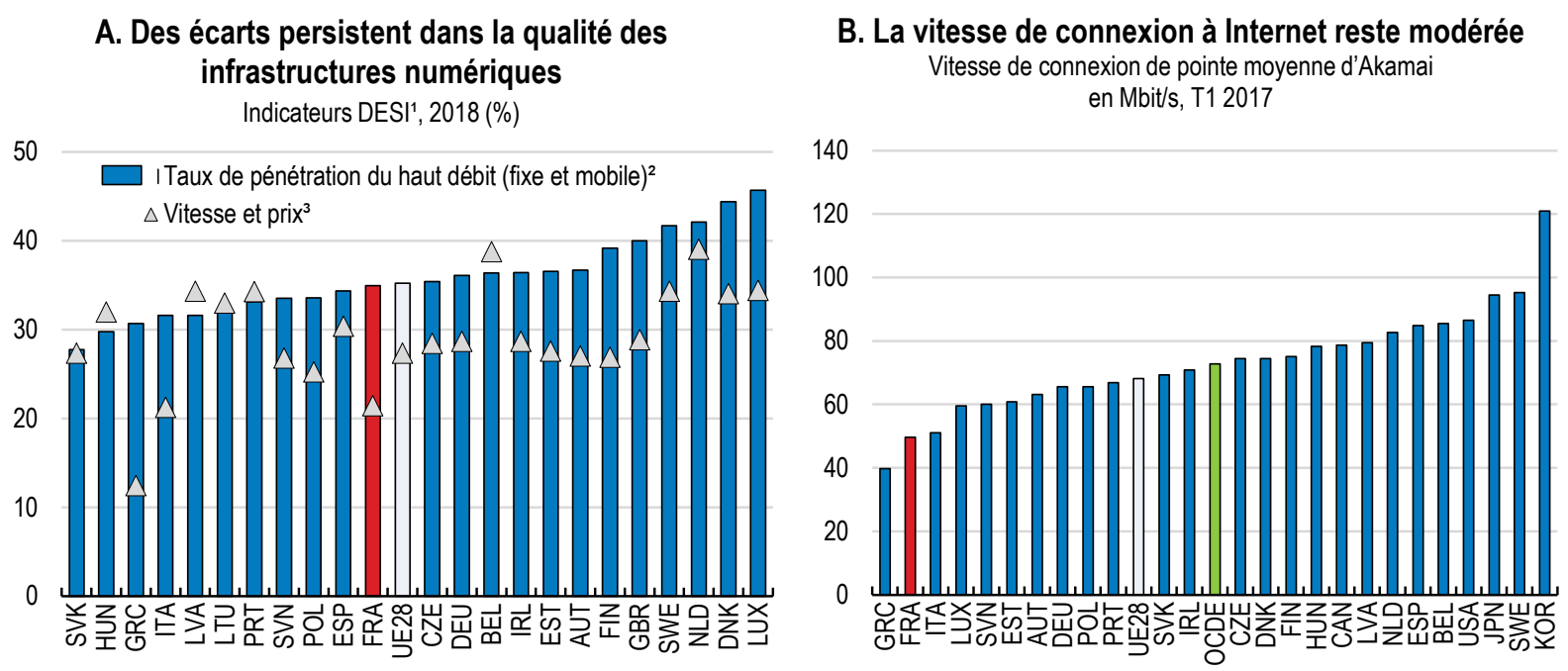

1. L'indicateur DESI (pour Digital Economy and Society Index) est un indice composite correspondant à la moyenne pondérée de cinq grandes dimensions portant sur la connectivité, le capital humain, l'utilisation de l'internet, l'intégration des technologies numériques et les services publics numériques. Plus sa valeur est élevée, meilleure est la performance.

2. Score cumulé des indicateurs fixe et mobile haut débit correspondant à la dimension « connectivité » de l'indicateur DESI.

3. Score cumulé des indicateurs de la dimension « connectivité » de l'indicateur DESI portant sur l'indice des prix du haut débit, du très haut débit et de l'ultra-haut débit.

Source : Commission européenne (2018), « Digital Economy and Society Index 2018 », disponible à l'adresse https://ec.europa.eu/digital-single-market/digital-economy-and-society-index-desi ； Akamai (2017), " Akamai's state of the Internet report: Q1 2017 report », https://www.akamai.com.

\section{StatLink तils https://doi.org/10.1787/888933952955}

Il serait judicieux de poursuivre les efforts de réexamen du cadre réglementaire, afin de s'assurer que le secteur privé est incité de manière satisfaisante à investir dans les infrastructures à très haut débit. Les résultats médiocres de la France en termes de vitesse de connexion à internet dans les classements internationaux laissent à penser que les incitations à l'investissement privé dans les infrastructures à haut débit ont pu laisser à désirer par le passé. Pour favoriser le développement des infrastructures à haut débit, l'Autorité de régulation des communications électroniques et des postes (ARCEP) encourage le partage des réseaux, afin d'éviter qu'il soit nécessaire de dupliquer les infrastructures, et incite au co-investissement dans les infrastructures à haut débit. En outre, en janvier 2018, l'État a accepté de geler le montant des redevances qui lui sont versées par 
les opérateurs privés de téléphonie mobile au titre de la location des réseaux, et les licences d'exploitation des fréquences mobiles attribuées à ces opérateurs ne seront pas renégociées en 2021, contrairement à ce qui était prévu initialement. En contrepartie, les opérateurs privés se sont engagés à étendre leur couverture mobile à des zones rurales et éloignées prédéfinies, et l'ARCEP veille au respect de ces engagements sur la base d'un suivi trimestriel.

À l'avenir, il pourrait être fructueux pour garantir la disponibilité d'infrastructures de réseau de haute qualité de reproduire ce modèle, consistant à réduire les redevances d'exploitation des opérateurs qui s'engagent à réaliser des investissements productifs - dans les réseaux de cinquième génération $(5 \mathrm{G})$, par exemple. Les pouvoirs publics ne devraient pas fixer les prix auxquels sont attribuées les fréquences du spectre hertzien dans le but de maximiser les recettes qu'ils en retirent, mais en s'appuyant sur des mécanismes de marché garantissant un taux de rendement adéquat sur la base des valeurs de marché. Par ailleurs, il faudrait réexaminer périodiquement le cadre réglementaire afin de s'assurer qu'il favorise les investissements nécessaires pour atteindre les objectifs des pouvoirs publics, sur la base d'évaluations transparentes fondées sur des données probantes (OCDE, 2017f).

\section{Tirer parti de l'investissement dans le numérique pour améliorer l'efficience du secteur public}

Accroître l'investissement public dans les technologies numériques pour favoriser leur utilisation par l'administration publique peut permettre aux pouvoirs publics, aux citoyens et aux entreprises de réaliser des économies, et améliorer l'efficacité de l'action publique. Les performances de la France en matière d'administration en ligne correspondent peu ou prou à la moyenne de l'UE (Graphique 18), ce qui laisse entrevoir des marges de progression. L'utilisation limitée de l'administration en ligne tient en partie aux choix des utilisateurs finals, ce qui laisse à penser que les campagnes de promotion de la transmission de formulaires en ligne pourraient être renforcées, et il serait bon d'accroître le recours aux formulaires pré-remplis. En outre, dans certaines fonctions des administrations publiques, telles que l'administration fiscale, les systèmes d'information sont en partie obsolètes, l'investissement dans les technologies numériques ayant diminué de plus de moitié depuis 2009 (Cour des comptes, 2018a). Une utilisation stratégique des investissements dans le numérique, par exemple pour mutualiser les prestations de services publics fournies en face-à-face, pourrait contribuer à la réalisation de gains de productivité dans l'administration publique. À cet égard, il convient de s'assurer que le montant alloué au fonds de transformation pour l'action publique est adéquat.

Le renforcement de l'administration numérique prévu par le Grand plan d'investissement (Encadré 2) bénéficierait également d'une accentuation des efforts déployés pour offrir des perspectives de carrière attractives aux spécialistes des technologies de l'information (Algan, Bacache et Perrot, 2016). Attirer des spécialistes des technologies de l'information ou de la science des données du secteur privé ou du monde universitaire, afin qu'ils apportent leurs compétences au service public dans le cadre de missions de courte durée, comme cela se fait aux États-Unis avec le programme de bourses présidentielles pour l'innovation (Presidential Innovation Fellowship), pourrait favoriser l'innovation en matière d'administration numérique. Les initiatives visant à renforcer la diffusion des technologies numériques dans l'administration publique progressent - on peut citer à cet égard beta.gouv.fr, un incubateur de projets axés sur la transformation numérique des services publics - mais il sera essentiel d'accélérer leur développement pour récolter pleinement les fruits des outils numériques dans l'administration publique. 


\section{Graphique 18. La pénétration des technologies numériques dans l'administration publique s'améliore}

Indice relatif à l'économie et à la société numériques (DESI) ${ }^{1}$, des valeurs plus élevées correspondent à de meilleures performances

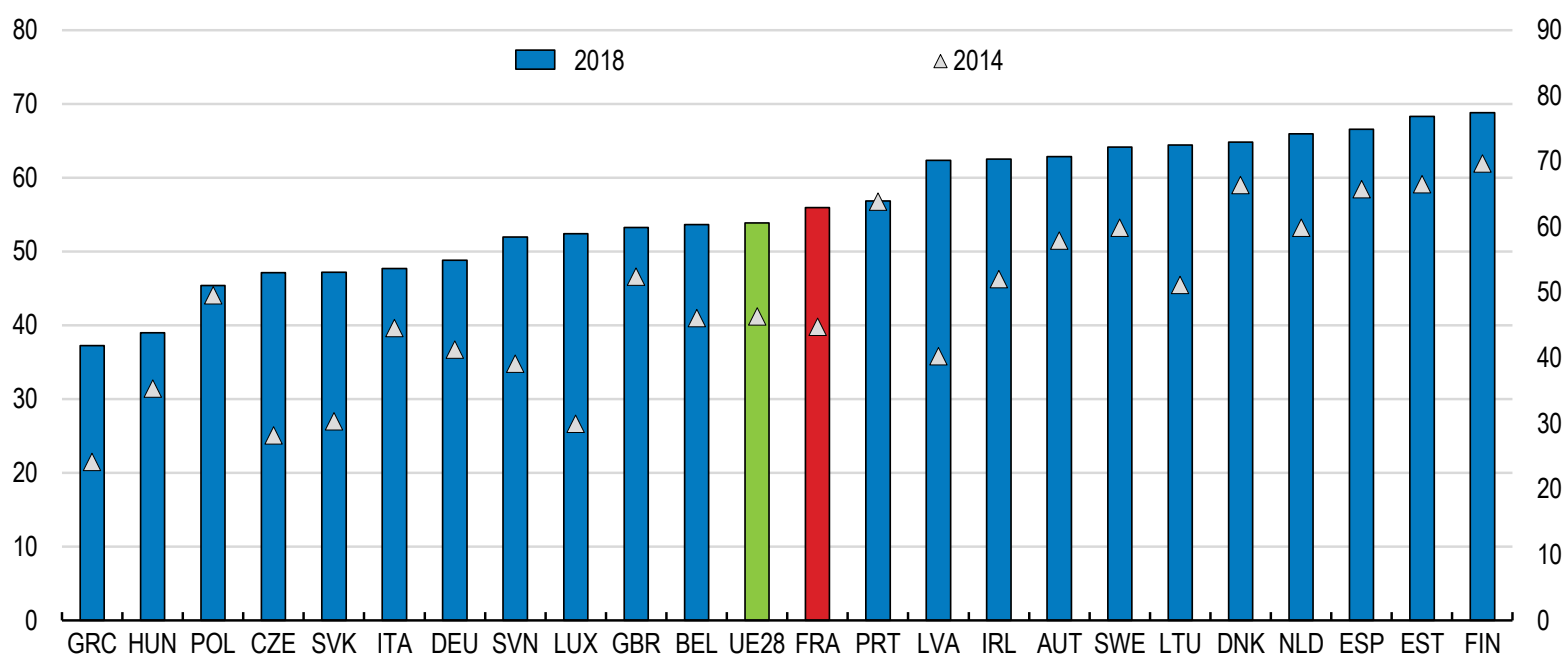

1. Sous-dimension « services publics numériques » de l'indicateur DESI correspondant à la moyenne pondérée des indicateurs normalisés suivants : utilisateurs des services publics numériques, utilisation de formulaires pré-remplis, achèvement des services en ligne et mise à disposition de données ouvertes.

Source : Commission européenne (2018), « Digital Economy and Society Index 2018 », disponible à l'adresse https://ec.europa.eu/digital-single-market/digital-economy-and-society-index-desi.

StatLink त्गाडs https://doi.org/10.1787/888933952974

\section{Tirer le meilleur parti de l'investissement public dans la $R \& D$}

Les dépenses de recherche-développement (R\&D) de la France correspondent peu ou prou à la moyenne de l'OCDE, mais elles sont loin de l'objectif fixé dans la stratégie Europe 2020, selon laquelle $3 \%$ du PIB de l'UE devrait être investi dans la R\&D en 2020 (Graphique 19, partie A). Les crédits budgétaires publics affectés aux activités de R\&D sont similaires à la moyenne de l'OCDE (Graphique 5). Les dépenses de R\&D des entreprises et la qualité de la production scientifique, évaluée à partir de mesures bibliométriques, sont également comparables aux moyennes de l'OCDE, même si les aides publiques à l'innovation des entreprises sont considérables (Graphique 19, parties B et C).

Le système public de recherche français se caractérise par le rôle important des organismes publics de recherche, qui occupent de longue date une place centrale dans la réalisation des activités publiques de recherche, et qui ont bénéficié en 2015 de la moitié environ des dépenses à destination de la recherche publique. Cette dualité du système public de recherche - fondé à la fois sur les organismes publics de recherche et les universités - est une source de complexité qui tire vers le haut les coûts de fonctionnement et peut entraver la répartition efficiente des ressources, ce qui appelle une poursuite des efforts de réforme (OCDE, 2014a). En vue de remédier à certains de ces problèmes, des réformes engagées au cours des années 2000 ont affirmé l'autonomie des universités et un organisme de financement de la recherche sous forme d'appels à projets a été mis en place. Par ailleurs, des mesures visent à améliorer l'articulation entre universités et organismes publics de recherche, dont le rôle national est renforcé, sur les sites où ils coopèrent. 
Graphique 19. Les dépenses de recherche-développement (R\&D) correspondent peu ou prou à la moyenne de l'OCDE

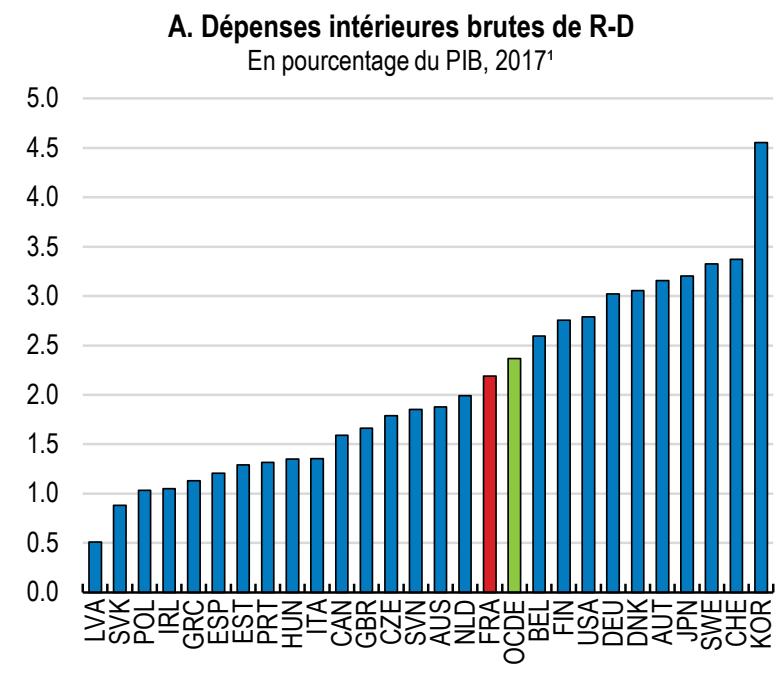
C. Aides publiques à la R-D des entreprises
En pourcentage du PIB, 2016

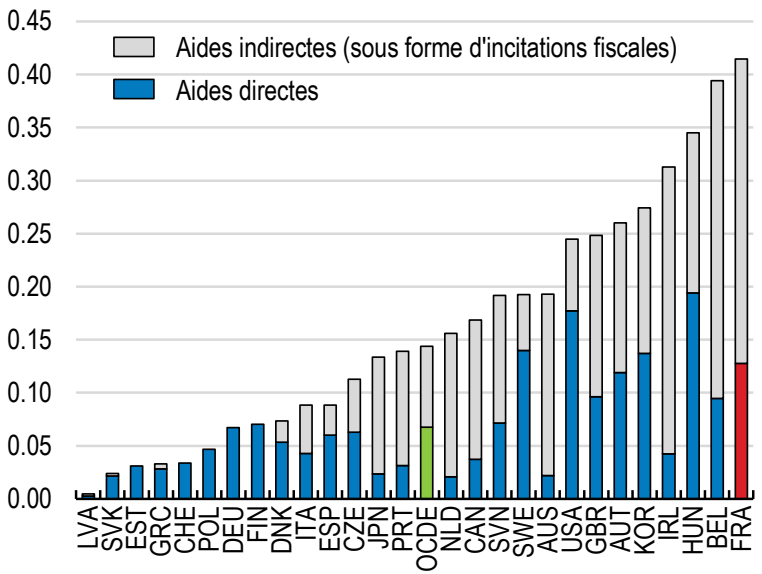

B. Dépenses de R-D des entreprises En pourcentage du PIB, $2017^{1}$

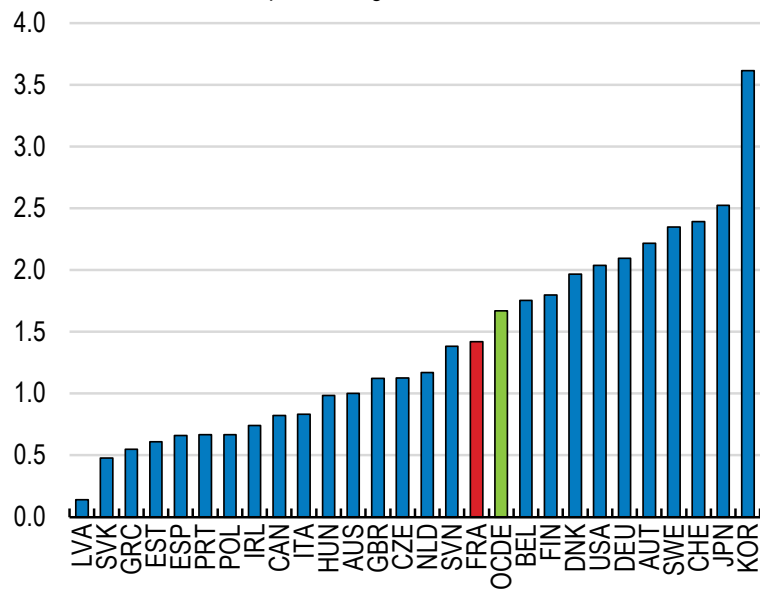

\section{Part des publications les plus citées au niveau} international $^{3}$
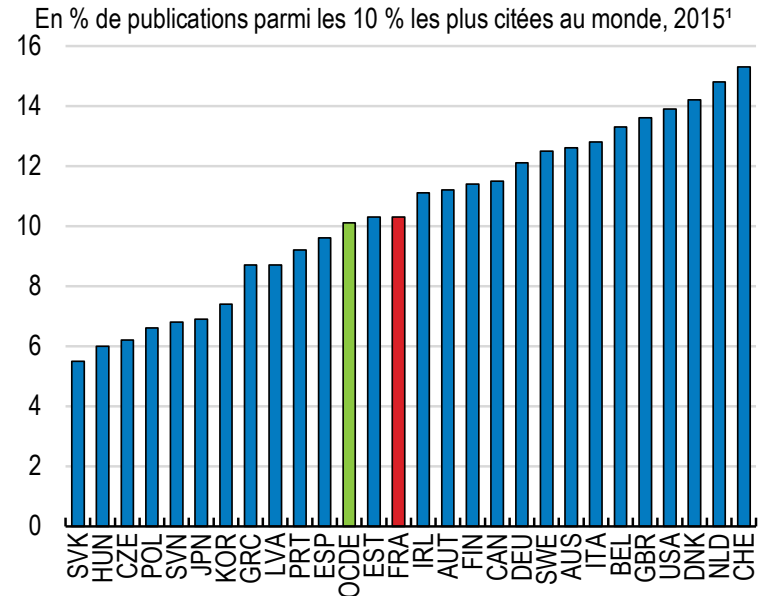

1. Ou dernière année connue.

2. Moyenne simple des données relatives aux pays membres de l'OCDE publiant des données pour les deux dimensions de cet indicateur. On ne dispose pas de données sur les aides indirectes dans le cas de la Pologne. 3. Part de la production scientifique des établissements de recherche nationaux figurant parmi les $10 \%$ de publications les plus citées dans le domaine scientifique correspondant, sur la base d'un comptage fractionnaire. Source : OCDE (2019), Statistiques OCDE de la recherche et développement et Indicateurs de l'OCDE sur les incitations fiscales à la $R \& D$ (bases de données) ; OCDE (2017), Science, technologie et industrie : Tableau de bord de l'OCDE 2017, Éditions OCDE, Paris.

\section{StatLink त्गाड़ https://doi.org/10.1787/888933952993}

Des plans d'investissement ponctuels, les programmes d'investissement d'avenir (PIA) (Encadré 2), ont également attribué des fonds par mise en concurrence aux universités et aux laboratoires de recherche pour favoriser l'émergence d'universités de rang mondial et restructuré le système de transferts de technologies pour encourager la commercialisation des résultats des activités publiques de recherche. Les PIA ont également considérablement étoffé les aides publiques à l'innovation des entreprises, et en parallèle avec le dernier plan 
d'investissement, un fonds d'investissement de 10 milliards d'euros destiné à accélérer le développement des jeunes entreprises à forte intensité technologique (deep tech) a été mis en place (Encadré 2).

Une simplification de la gouvernance des unités mixtes de recherche (UMR), qui sont à la pointe des activités de recherche, contribuerait à favoriser une répartition efficiente des ressources. La gouvernance de ces UMR, qui se trouvent généralement dans des universités, est complexe, dans la mesure où leur budget et leur personnel dépendent à la fois de l'université et d'un ou plusieurs organismes publics de recherche, notamment le Centre national de la recherche scientifique (CNRS). Cela peut potentiellement déboucher sur une répartition incohérente des ressources, car les unités de recherche ayant obtenu des financements par appels à projets peuvent ne pas être en mesure d'attirer des personnes expérimentées pour développer ceux-ci. Par ailleurs, la copropriété des droits de propriété intellectuelle, détenus à la fois par les universités et les organismes publics de recherche, au sein des UMR allonge et complique le processus de commercialisation des résultats de la recherche. Le projet de loi «PACTE » renforce et élargit le principe du mandataire unique qui permettra aux entreprises de disposer d'un unique interlocuteur lorsqu'elles souhaitent exploiter les résultats innovants issus d'une UMR. Par ailleurs, continuer les efforts visant à limiter au strict nécessaire le nombre de tutelles des UMR et à renforcer le partage de la stratégie scientifique et de moyens entre les tutelles contribuerait à simplifier la gouvernance des UMR et à favoriser une répartition efficiente des ressources.

Poursuivre les réformes pour renforcer l'autonomie des universités en termes de gestion et de rémunération de leur personnel contribuerait à récolter pleinement les fruits des précédents PIA. Il serait notamment judicieux de supprimer la procédure de qualification de droit commun obligatoire au niveau national pour pouvoir postuler à un poste d'enseignant-chercheur, dans la mesure où elle peut faire obstacle aux recrutements internationaux et à la recherche interdisciplinaire. En outre, dans le cadre des PIA, un groupe d'universités triées sur le volet s'est vu attribuer des dotations conséquentes au titre d'initiatives d'excellence (IDEX), qui visaient notamment à stimuler les recrutements de scientifiques travaillant à l'étranger. Il s'agissait là d'une mesure bienvenue, la France ayant connu des départs importants d'auteurs scientifiques depuis 2002, sachant que cette évolution s'est accélérée dernièrement (Graphique 20). Néanmoins, le recrutement de scientifiques grâce à ces dotations semble avoir été limité, ce qui s'explique en partie par la nécessité pour les universités d'assimiler pleinement ce nouveau mécanisme de financement, ainsi que par la période probatoire qui conditionne la pérennisation de ces financements. L'élaboration d'un programme tel que le dispositif canadien «Chaires de recherche Canada $150 »$, qui attribue des financements supplémentaires aux universités sous réserve qu'elles attirent des chercheurs en poste à l'étranger, pourrait compléter utilement les dotations destinées à renforcer les recrutements internationaux de chercheurs prometteurs ou expérimentés. 


\section{Graphique 20. Flux internationaux nets d'auteurs scientifiques}

Différence entre les entrées et les sorties annuelles (mesurées sur la base d'un comptage fractionnaire), en pourcentage des flux totaux

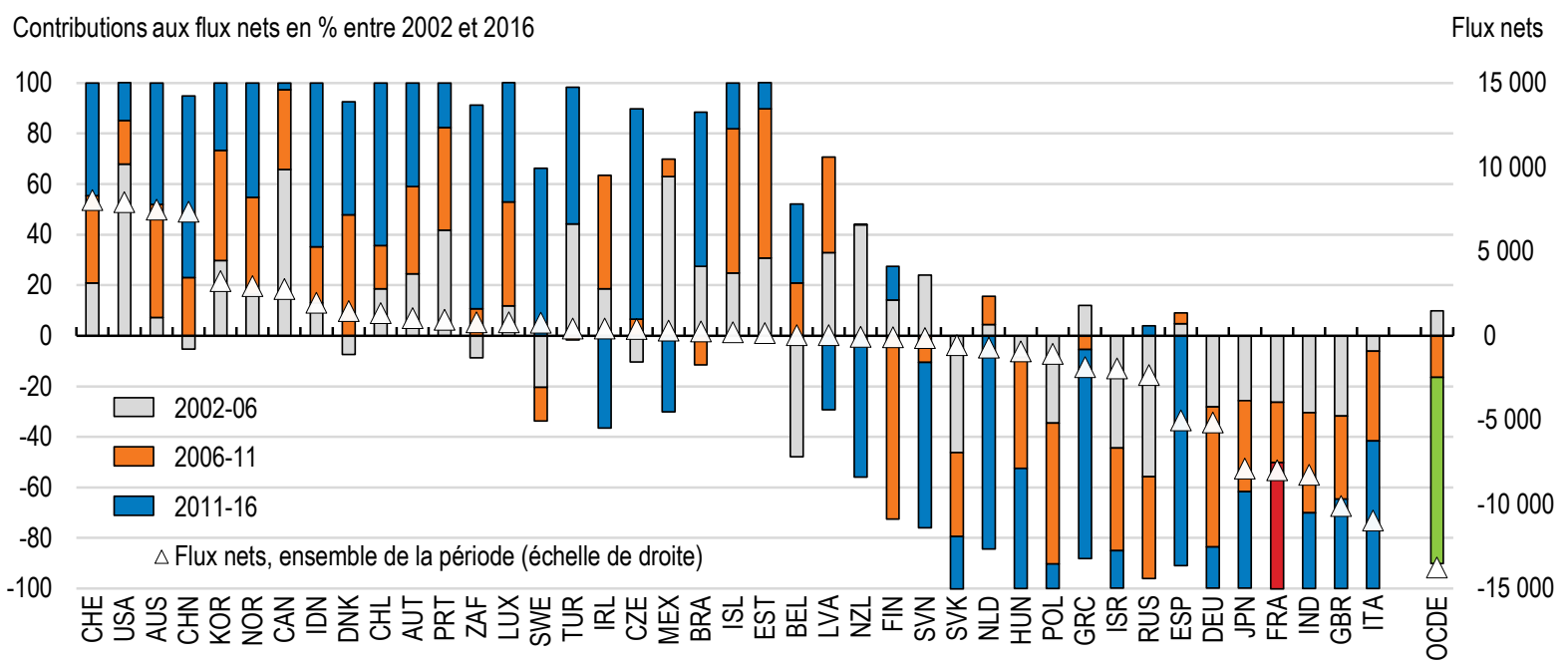

Note: Ces estimations reposent sur les différences entre les entrées et les sorties implicites d'auteurs scientifiques pour l'économie considérée, telles qu'indiquées par le changement d'affiliation principale d'un auteur donné référencé dans la base de données Scopus, au cours de la période pendant laquelle cet auteur a eu des publications référencées. Le graphique décompose les flux nets enregistrés année après année au cours de la période considérée, dans une sélection d'économies. Une entrée est comptabilisée pour une année t et une économie e si l'on constate qu'un auteur auparavant affilié à une autre économie est affilié pour la première fois l'année t à un établissement de l'économie e. De même, une sortie est enregistrée lorsqu'on constate qu'un auteur affilié à l'économie e au cours de l'année précédente est affilié pour la première fois l'année $\mathrm{t}$ à une autre économie. Si un auteur est affilié à plusieurs économies, on effectue un comptage fractionnaire. Si, au cours d'une année donnée, un auteur a produit plusieurs publications, la dernière d'entre elles est utilisée comme référence, tandis que les autres sont ignorées.

Source: OCDE (2018), Science, technologie et industrie : Tableau de bord de l'OCDE 2017, Éditions OCDE, Paris.

StatLink त्ञाज https://doi.org/10.1787/888933953012

\section{Le financement concurrentiel de la recherche progresse, mais reste faible}

Comparer la part du financement concurrentiel de la recherche entre pays est un exercice délicat, compte tenu du manque de données comparables (OCDE, 2018f). Néanmoins, la part des financements concurrentiels de la recherche semble faible en France par comparaison avec les autres pays, sur la base des données disponibles (Graphique 21, partie A). Créée en 2005, l'Agence nationale de la recherche (ANR) est le principal organisme chargé de financer par appels à projets les universités et les organismes publics de recherche, mais aussi des projets de recherche réalisés par des entreprises, qui représentent environ $20 \%$ des fonds attribués par l'ANR. Des financements sont aussi attribués par mise en concurrence des unités de recherche via les PIA, même si ces derniers constituent des mécanismes de financement exceptionnels, alors que le système de financement concurrentiel administré par l'ANR est régulier. L'ANR est l'un des mécanismes de financements concurrentiels aux côtés de ceux gérés par d'autres acteurs tels que les ministères ou les organismes publics de recherche.

Il est nécessaire de veiller à ce que des ressources suffisantes et prévisibles soient affectées au financement concurrentiel de la recherche publique. Le budget de l'ANR s'est 
sensiblement réduit entre 2009 et 2015 (Graphique 21, partie B), et le taux de succès aux appels à projets a diminué au fil du temps jusqu'à un point bas de $10.6 \%$ en 2014 , qui semble trop faible pour sélectionner les projets de recherche eu égard aux difficultés inhérentes à cette sélection. Le temps consacré par les scientifiques à la préparation des demandes de financement est également considérable, voir Herbert, Barnett et Graves, 2013 dans le contexte australien. Cela suggère qu'un financement suffisant de l'ANR est nécessaire pour préserver les avantages du système de financement sur projet en termes de qualité et de pertinence des projets de recherche, tout en réduisant au minimum ses inconvénients liés à l'incapacité des chercheurs et des laboratoires de planifier leurs activités à long terme. Par conséquent, les récentes augmentations du budget de l'ANR dans le but d'augmenter les taux de succès au-delà de $20 \%$, conjuguée à l'initiative en cours visant, au travers d'une loi de programmation pluriannuelle de la recherche, à définir une trajectoire financière pluriannuelle pour s'assurer de la prévisibilité du financement de la recherche publique et en tirer le meilleur parti, sont bienvenues. Par ailleurs, il serait bon que l'ANR lance également des appels à projets de recherche complémentaires à ceux du programme Horizon 2020 de l'UE (mais non identiques), afin de garantir la diversité des projets de recherche. Assurer des financements appropriés pour des projets sans thématique précise, mais qui visent à récompenser l'excellence des travaux de recherche, semble également opportun.

Graphique 21. Le financement sur projet des activités de recherche-développement (R\&D) est limité
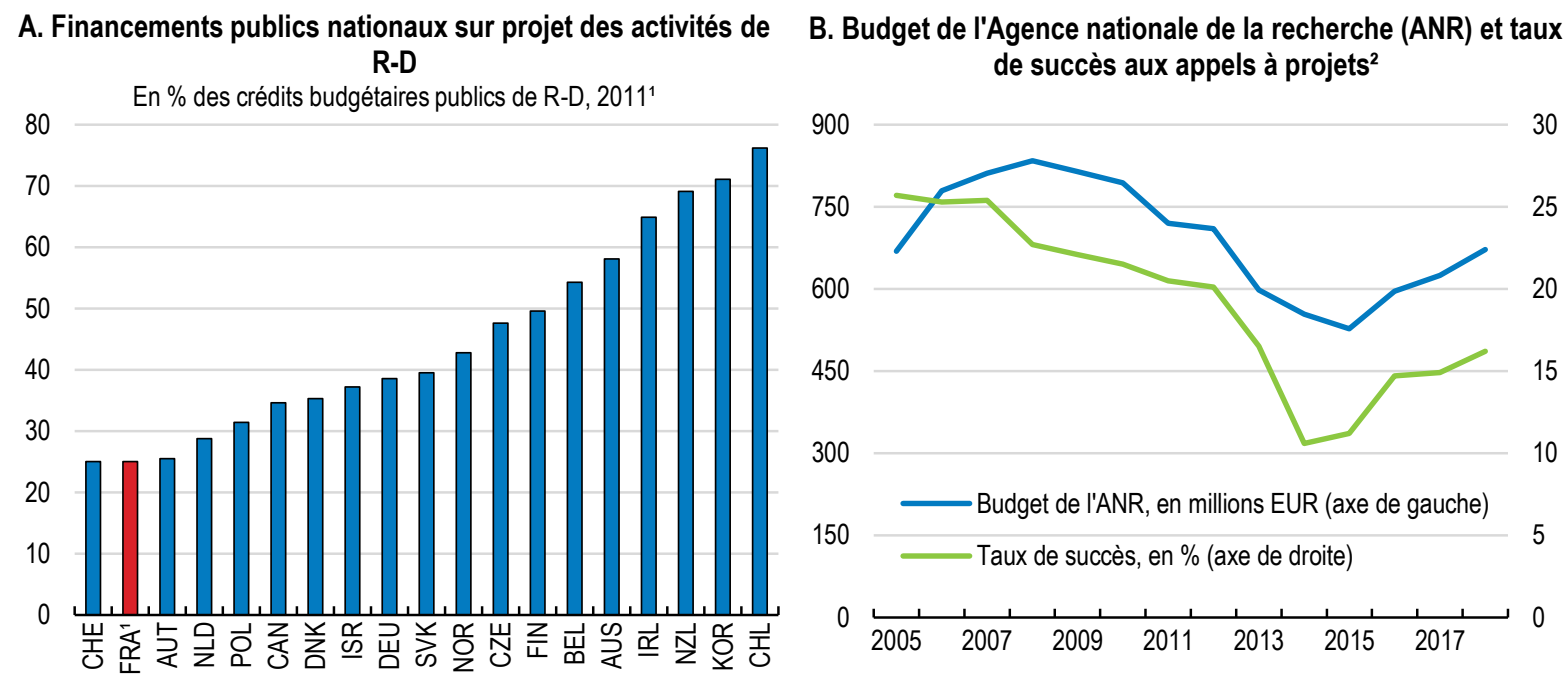

1. Le chiffre relatif à la France - environ $25 \%$ - inclut les mécanismes de financement sur projet internes aux organismes publics de recherche et aux universités, de sorte qu'il diffère largement du chiffre d'Eurostat (8\%), qui recouvre uniquement les financements sur projet attribués par les organismes nationaux de financement par mise en concurrence.

2. Le budget de l'ANR représenté dans la partie B est le budget d'intervention global (hors frais de fonctionnement).

Source: OCDE, 1ère et 2ème enquêtes expérimentales sur les modes de financement de la recherchedéveloppement (R\&D) fondées sur les crédits budgétaires publics de recherche-développement (CBPRD), mentionnées dans OCDE (2018); et ministère français de l'Enseignement supérieur, de la Recherche et de l'Innovation. 


\section{Les aides à l'innovation des entreprises ont sensiblement augmenté}

Les aides à l'innovation des entreprises ont considérablement augmenté au cours des dix dernières années. Il existe de nombreuses aides directes destinées à favoriser l'innovation des entreprises. De plus, le soutien apporté à l'innovation du secteur privé prend essentiellement la forme d'un crédit d'impôt en faveur de la $R \& D$ qui figure parmi les plus généreux de la zone OCDE (Graphique 19, partie C). Si les dépenses de R\&D des entreprises correspondent à la moyenne de l'OCDE, l'intensité de R\&D des entreprises françaises devient nettement supérieure une fois corrigée de la structure industrielle, la France étant spécialisée dans des secteurs à moindre intensité de R\&D (Graphique 22). Le système d'aides à l'innovation des entreprises est relativement complexe, dans la mesure où le nombre de dispositifs d'aide directe à l'innovation a été multiplié par deux au cours des quinze dernières années (MESRI, 2018). Les PIA ont également débouché sur la mise en place de nouveaux organismes destinés à renforcer la coopération entre les entreprises et les milieux scientifiques qui font en partie doublon avec le précédent système de transferts de technologies.

\section{Graphique 22. L'intensité de recherche-développement (R\&D) des entreprises est plus forte une fois corrigée de la structure d'activités}

En pourcentage de la valeur ajoutée par secteur, $2015^{1}$

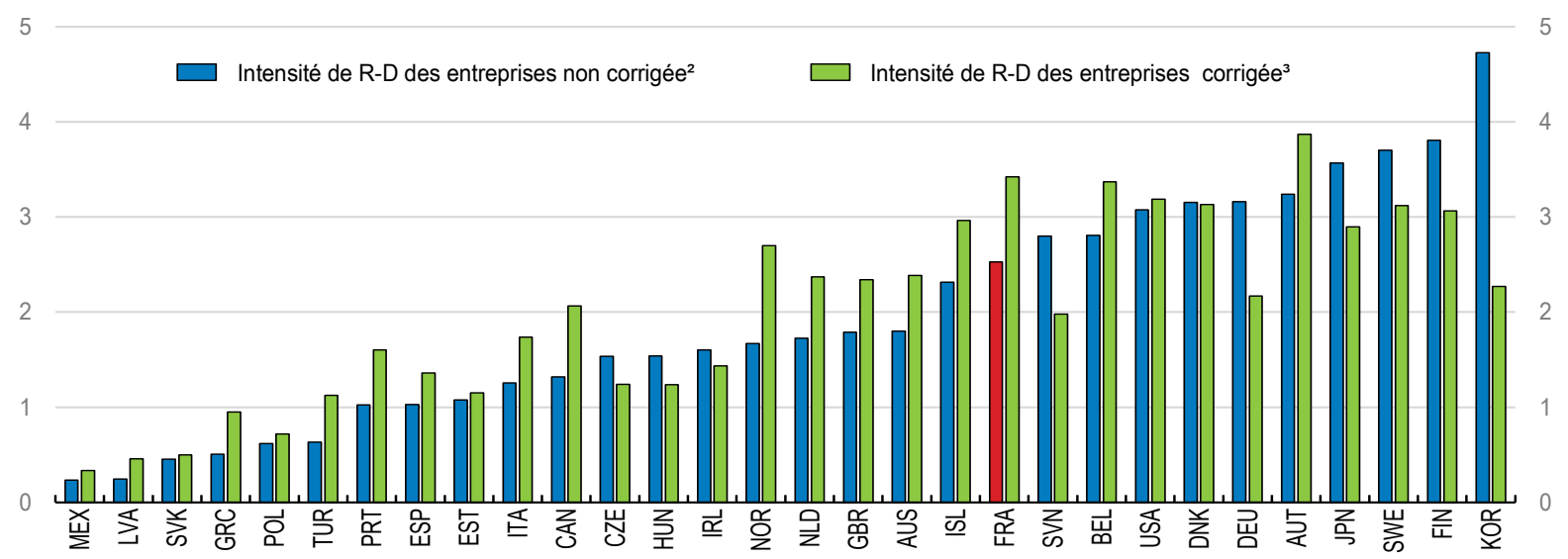

1. Les données se rapportent à 2014 pour le Danemark, les États-Unis, la Finlande, la Hongrie, l'Italie, les PaysBas, la Pologne, le Portugal, la Slovénie et le Royaume-Uni ; et à 2013 pour l'Australie l'Autriche, la Belgique, le Canada, la Corée, la France, la Grèce, l'Irlande et la Suède.

2. Les statistiques de R\&D sont présentées en pourcentage de la valeur ajoutée du secteur des entreprises, estimée comme la valeur ajoutée de toutes les branches d'activité hormis les secteurs suivants : Activités immobilières (division 68 de la Classification internationale type, par industrie, de toutes les branches d'activité économique (CITI) Révision 4); Administration publique et défense ; sécurité sociale obligatoire (division 84 de la CITI Rév. 4) ; Éducation (division 85 de la CITI Rév. 4) ; Santé et activités d'action sociale (divisions 86 à 88 de la CITI Rév. 4) ; et Activités des ménages privés employant du personnel domestique (divisions 97 et 98 de la CITI Rév. 4).

3. L'intensité de R\&D des entreprises corrigée de la structure d'activités d'un pays donné est la moyenne pondérée des intensités de R\&D de ses différents secteurs d'activité (ratio des dépenses de R\&D à la valeur ajoutée de chaque secteur) calculée à partir de la structure d'activités des pays de l'OCDE considérés dans leur ensemble ; autrement dit, on utilise pour chaque pays les mêmes coefficients de pondération (correspondant à la part de chaque secteur dans la valeur ajoutée de la zone OCDE en 2015). L'intensité de R\&D des entreprises non corrigée est, par définition, une moyenne pondérée calculée en utilisant comme coefficients de pondération les parts effectives de chaque secteur dans la valeur ajoutée des entreprises du pays considéré.

Source: OCDE (2018), Science, technologie et industrie : Tableau de bord de l'OCDE 2017, Éditions OCDE, Paris. 
Il faudrait maintenant s'attacher avant tout à garantir la cohérence et la clarté des dispositifs existants d'aide à l'innovation des entreprises. Dans ce contexte, les autorités ont créé à l'été 2018 le Conseil de l'innovation, chargé de définir les grandes orientations de la politique de l'innovation, notamment en réexaminant les mesures en place dans un objectif de simplification. Les aides à l'innovation exigent une planification prudente ainsi qu'un soutien régulier et stable, ce qui demande une simplification progressive, qui devrait être fondée sur des évaluations ex post approfondies des dispositifs en place, permettant de concentrer les efforts déployés sur les plus utiles et d'éliminer ceux qui n'ont pas ou peu d'impact. Les aides à l'innovation reposent aussi dans une large mesure sur des plans d'investissement ponctuels, tels que les PIA, ce qui nuit à la stabilité des dispositifs retenus (Inspection générale des Finances, 2018); il faudrait donc déployer des efforts de planification budgétaire pluriannuelle des politiques d'innovation pour garantir la stabilité des mesures les plus efficaces.

\section{Recommandations visant à améliorer l'efficience de l'investissement public}

(Les recommandations soulignées sont reprises des Principales recommandations)

\section{Améliorer les conditions-cadres des opérations d'investissement}

- Prendre davantage en compte les considérations d'efficience socio-économique lors de la sélection des grands projets. Pour cela :

- faire plus d'efforts pour généraliser, chez toutes les parties prenantes, la pratique consistant à procéder à des évaluations socio-économiques avant d'effectuer des investissements importants, notamment en continuant à élargir à d'autres secteurs le cadre mis en place dans le secteur de la santé ;

- étendre les obligations d'évaluation socio-économique aux grands investissements effectués en totalité par les collectivités locales ;

- obliger les autorités concernées à prendre officiellement en compte les résultats de la contre-expertise des évaluations socio-économiques ex ante ;

- rendre les évaluations ex post obligatoires pour tous les types d'investissements importants.

- $\quad$ Renforcer la part de la maintenance des infrastructures dans l'investissement public.

Mobiliser le secteur privé pour la réalisation d'infrastructures

- Améliorer le recueil d'informations sur les performances financières et non financières des infrastructures en fonction des différents modèles de marchés publics pour permettre des prises de décision plus éclairées sur la participation du secteur privé à la réalisation d'infrastructures.

- Accroître le nombre des projets prêts à financer dans les programmes sectoriels à long terme pour attirer les investisseurs privés.

\section{Renforcer les capacités d'investissement public des collectivités locales}

- Encourager le transfert complet des responsabilités en matière d'infrastructures locales et d'urbanisme aux intercommunalités.

- Rationaliser les fonds de péréquation verticale versés aux communes, développer davantage la péréquation horizontale pour s'attaquer aux disparités en matière de collecte de recettes fiscales, et donner un rôle plus important aux approches basées sur le coût unitaire standard dans la détermination des concours financiers de l'État aux collectivités territoriales. 


\section{Politiques visant à améliorer l'efficience de l'investissement dans des secteurs spécifiques}

- Mettre en place des mécanismes de correction du soutien public aux politiques de l'énergie et de lutte contre le changement climatique lorsque les indicateurs annuels dévient trop fortement par rapport aux objectifs.

- Développer les possibilités d'interconnexion entre les réseaux électriques européens.

- Continuer de promouvoir la tarification efficace de l'usage des infrastructures de transport, par exemple au moyen de redevances de congestion ou basées sur la distance parcourue, pour développer des modes de transport alternatifs et réduire la pollution de l'air.

- Améliorer le recueil d'informations sur la qualité des réseaux d'infrastructures de transport pour concentrer les investissements d'entretien là où ils sont le plus nécessaires.

- Utiliser l'investissement numérique pour obtenir des gains de productivité dans l'administration publique.

- $\quad$ Renforcer l'autonomie des universités en matière de définition des programmes et de gestion du personnel, en tenant compte de leur mission de service public. Continuer d'augmenter le budget de l'Agence nationale de la recherche.

- Continuer la simplification de la gestion des unités de recherche et des droits de propriété intellectuelle.

\section{Bibliographie}

Agence internationale de l'énergie (2017), Energy Technology Perspectives 2017, Agence internationale de l'énergie, Paris, http://www.iea.org/etp2017.

Agence internationale de l'énergie (2016), Energy Policy of IEA Countries: France 2016 Review, http://www.oecd.org/publications/energy-policies-of-iea-countries-france-2016-9789264279445en.htm.

Algan, Y., M. Bacache et A. Perrot (2016), « Administration numérique », Les notes du conseil d'analyse économique, vol. 34.

Allain-Dupré, D. (2018), « Assigning responsibilities across levels of government : Trends, challenges and guidelines for policy-makers », OECD Working Papers on Fiscal Federalism, no. 24, Éditions OCDE, Paris, https://dx.doi.org/10.1787/f0944eae-en.

ARAFER (2018), Avis relatif à la mise en æuvre au titre de l'année 2017 du contrat pluriannuel de performance conclu entre l'Etat et SNCF Réseau pour la période 2017 - 2026, Paris, http://www.arafer.fr/wp-content/uploads/2018/05/avis-2018-035-du-14-mai-2018-contratpluriannuel-de-performance-conclu-entre-letat-et-sncf-reseau-version-publique.pdf.

Araújo, S. and D. Sutherland (2010), "Public-Private Partnerships and Investment in Infrastructure", OECD Economics Department Working Papers, No. 803, OECD Publishing, Paris, http://dx.doi.org/10.1787/5km7jf6q8f0t-en.

Assemblée Nationale (2018), Commission d'enquête sur la sûreté et la sécurité des installations nucléaires, Rapport, Paris, http://www.assemblee-nationale.fr/15/pdf/rap-enq/r1122-tI.pdf. 
Autorité de la concurrence (2017), Avis $n^{\circ}$ 17-A-04 du 20 mars 2017 concernant un projet de décret relatif au transport particulier de personnes, http://www.autoritedelaconcurrence.fr/pdf/avis/17a04.pdf.

Autorité de sûreté nucléaire (2017), Avis $n^{\circ}$ CODEP-CLG-2017-022588, Rapport, Montrouge, https://www.asn.fr/Reglementer/Bulletin-officiel-de-l-ASN/Installations-nucleaires/Avis/Avis-nCODEP-CLG-2017-022588-du-President-de-1-ASN-du-8-juin-2017.

Banque européenne d'investissement (2017), EIB Investment Survey 2017 - Municipal Infrastructure, Luxembourg.

Bartolini, D. (2015), « Municipal Fragmentation and Economic Performance of OECD TL2 Regions », Documents de travail de l'OCDE sur le développement régional, no. 2015/2, Éditions OCDE, Paris, https://dx.doi.org/10.1787/5jrxqs60st5h-en.

Bergère, F. (2016), « Ten years of PPP: An initial assessment », OECD Journal on Budgeting, vol. 2015/1.

Brosio, G. (2018), « Ambition beyond feasability? Equalization transfers to regional and local governments in Italy », http://documentos.fedea.net/pubs/dt/2018/dt2018-09.pdf.

CEREMA (2018), Synthèse des bilans ex post des projets routiers structurants, Rapport, Paris.

CGEDD et CGE (2018), Mission portant sur la transformation des aides à l'investissement du Fonds chaleur en aides à la production de chaleur renouvelable, http://www.cgedd.developpementdurable.gouv.fr/IMG/pdf/012040-01_rapport_cle23a9f2.pdf.

Climate Action Tracker (2018), The highway to Paris. Safeguarding the climate by decarbonising freight transport. CAT Decarbonisation Series, New Climate Institute, Ecofys, Climate Analytics, https://qz.com/1158107/the-german-postal-service-has-.

Commission européenne (2017), Third Report on the State of the Energy Union, Rapport, Bruxelles.

Conseil général de l'environnement et du développement durable (2017), Revue de dépenses : La voirie des collectivités territoriales, Rapport, Paris, http://cgedd.documentation.developpementdurable.gouv.fr/documents/cgedd/011055-01 rapport.pdf.

Cour des comptes (2018a), La DGFiP, dix ans après la fusion: Une transformation à accélérer, Rapport, Paris, https://www.ccomptes.fr/sites/default/files/2018-06/20180620-rapport-DGFip-10-ansapres-fusion.pdf.

Cour des comptes (2018b), Le processus de privatisation des aéroports de Toulouse, Lyon et Nice, Rapport, Paris, https://www.ccomptes.fr/system/files/2018-11/20181113-processus-privatisationaeroports-Toulouse-Lyon-Nice.pdf.

Cour des comptes (2018c), Le soutien aux énergies renouvelables, Rapport, Paris, https://www.ccomptes.fr/sites/default/files/2018-04/20180418-rapport-soutien-energiesrenouvelables.pdf.

Cour des comptes (2017a), Les insuffisances du cadre juridique et comptable applicable aux entreprises publiques locales, Rapport, Paris, https://www.ccomptes.fr/sites/default/files/2017-10/20170927refere-G64-17-0518E-cadre-comptable-entreprises-publiques-locales.pdf.

Cour des comptes (2017b), La situation du transport de marchandises par le groupe SNCF Mobilités, Référé, Paris, https://www.ccomptes.fr/sites/default/files/2017-09/20170914-refere-S2017-1999transport-marchandises-SNCF_0.pdf. 
Cour des comptes (2016a), Concours financiers de l'État et disparités de dépenses des communes et de leurs groupements, Rapport, Paris, https://www.ccomptes.fr/fr/publications/concours-financiers-deletat-et-disparites-de-depenses-des-communes-et-de-leurs.

Cour des comptes (2016b), La maintenance des centrales nucléaires, Rapport, Paris, https://www.ccomptes.fr/sites/default/files/EzPublish/04-maintenance-centrales-nucleaires-RPA2016Tome-1.pdf.

Cour des comptes (2015a), Les finances publiques locales : Rapport sur la situation financière et la gestion des collectivités territoriales et de leurs établissements publics, Rapport, Paris, https://www.ccomptes.fr/sites/default/files/EzPublish/20151013-rapport-finances-publiqueslocales.pdf.

Cour des comptes (2015b), Les partenariats public-privé des collectivités territoriales : des risques à maîtriser, Rapport, Paris, https://www.ccomptes.fr/sites/default/files/EzPublish/125-RPA2015partenariats-public-prive.pdf.

Cour des comptes (2014), La grande vitesse ferroviaire : un modèle porté au-delà de sa pertinence, Rapport, Paris, https://www.ccomptes.fr/sites/default/files/EzPublish/20141023 rapport_grande_vitesse_ferroviaire.p df.

Cour des comptes de l'Union européenne (2016), Le transport ferroviaire de marchandises dans l'UE: toujours pas sur la bonne voie, Rapport, Luxembourg, https://www.eca.europa.eu/en/Pages/DocItem.aspx?did=36398.

Cournède, B. et V. Ziemann (2019), Scoping paper on infrastructure, Document de travail du département des Affaires économiques de l'OCDE, à paraître.

Crozet, Y. (2013), « Performance in France: From Appraisal Methodologies to Ex-post Evaluation», Discussion Paper No. 2013-26, Forum international des transports, Paris, https://www.itfoecd.org/sites/default/files/docs/dp201326.pdf.

Dotterud, L. et K. Skollerud (2015), "Public Transport Services in Norway: Focus on Rural Areas", https://www.itf-oecd.org/sites/default/files/docs/dp201508.pdf.

Eliasson, J., L. Hultkrantz et L. Smidfelt Rosqvist (2009), « The Stockholm congestion - charging trial 2006: Overview of effects », Transportation Research Part A: Policy and Practice, vol. 43/3, pp. 240-250, https://doi.org/10.1016/j.tra.2008.09.007.

Forum international des transports (2017), «Transition to Shared Mobility : How large cities can deliver inclusive transport services », International Transport Forum Policy Papers, no. 33, Éditions OCDE, Paris, https://dx.doi.org/10.1787/b1d47e43-en.

Forum international des transports (2018), Private Investment in Transport Infrastructure: Dealing with Uncertainty in Contracts, https://www.itf-oecd.org/sites/default/files/docs/private-investmenttransport-infrastruture.pdf.

France Stratégie (2019), L'évaluation socioéconomique des projets immobiliers de l'enseignement supérieur et de la recherche, Rapport du groupe de travail présidé par Émile Quinet, https://www.strategie.gouv.fr/publications/levaluation-socioeconomique-projets-immobiliers-delenseignement-superieur-de-recherche.

Gouvernement (2018), Évaluation des grands projets d'investissements publics, https://www.performancepublique.budget.gouv.fr/sites/performance_publique/files/files/documents/jaunes2019/jaune2019 investissements publics.pdf. 
Gouvernement (2017a), Revue de dépenses de voirie des collectivités territoriales, https://www.interieur.gouv.fr/Publications/Rapports-de-l-IGA/Rapports-recents/Revue-de-depensesLa-voirie-des-collectivites-territoriales.

Gouvernement (2017b), Évaluation des grands projets d'investissements publics, https://www.gouvernement.fr/sites/default/files/contenu/piecejointe/2018/03/jaune2018_projets_investissements_publics.pdf.

Herbert, D., A. Barnett et N. Graves (2013), «Funding: Australia's grant system wastes time », Nature, vol. 495, p. 314, https://doi.org/10.1038/495314d.

HM Treasury (2015), Public sector business cases using the five case model: Updated guidance.

Inspection générale des Finances (2018), Les aides à l'innovation, Rapport, Paris, http://www.igf.finances.gouv.fr/files/live/sites/igf/files/contributed/IGF\%20internet/2.RapportsPublic s/2018/rapport-innovation.pdf.

Inspection générale des Finances (2016), Évaluation des procédures d'évaluation socio-économique des projets d'investissement public, Rapport, Paris,

http://www.igf.finances.gouv.fr/files/live/sites/igf/files/contributed/IGF\%20internet/2.RapportsPublic s/2016/2016-M-058.pdf.

Kim, J. et S. Dougherty (2018), Fiscal Decentralisation and Inclusive Growth, OECD Fiscal Federalism Studies, Éditions OCDE, Paris/KIPF, Séoul, https://dx.doi.org/10.1787/9789264302488-en.

Marchionni, E., C. Pollastri et A. Zanardi (2017), Fabbisogni standard e capacità fiscali nel sistema perequativo dei Comuni, http://www.upbilancio.it/wp-content/uploads/2017/02/Nota-1_2017.pdf.

MESRI (2018), Installation du conseil de l'innovation, Ministère de l'Enseignement supérieur, de la Recherche et de l'Innovation, http://www.enseignementsup-recherche.gouv.fr/pid25439cid132970/installation-du-conseil-de-l-innovation.html.

Ministère de la Transition écologique et solidaire (2018), Réseau routier national non concédé : résultats d'audits, Paris, https://www.ecologiquesolidaire.gouv.fr/sites/default/files/2018.07.10_dossier_reseau_routier.pdf.

Observatoire des services publics d'eau et d'assainissement (2017), Panorama des services et de leur performance en 2014, http://www.eaufrance.fr/IMG/pdf/spea2014_201705.pdf.

OCDE (2018a), Science, technologie et innovation: Perspectives de l'OCDE 2018 (version abrégée) : S'adapter aux bouleversements technologiques et sociétaux, Éditions OCDE, Paris, https://dx.doi.org/10.1787/sti_in_outlook-2018-fr.

OCDE (2018b), Monitoring Report: Implementation of the Recommendation of the Council on Effective Public Investment across Levels of Government, Éditions OCDE, Paris, à paraître.

OCDE (2018c), Developing Robust Project Pipelines for Low-Carbon Infrastructure, Green Finance and Investment, Éditions OCDE, Paris. https://doi.org/10.1787/9789264307827-en .

OCDE (2018d), Subnational Public-Private Partnerships : Meeting Infrastructure Challenges, OECD Multi-level Governance Studies, Éditions OCDE, Paris, https://dx.doi.org/10.1787/9789264304864en.

OCDE (2018e), Bridging the Rural Digital Divide, OECD Digital Economy Papers, vol. 265.

OCDE (2018f), Effective operation of competitive research funding systems, OECD Science, Technology and Industry Policy Papers, Editions OCDE, Paris, https://doi.org/10.1787/23074957. 
OCDE (2017a), The Size and Sectoral Distribution of State-Owned Enterprises, Éditions OCDE, Paris, https://dx.doi.org/10.1787/9789264280663-en.

OCDE (2017b), Getting Infrastructure Right : A framework for better governance, Éditions OCDE, Paris, https://dx.doi.org/10.1787/9789264272453-en.

OCDE (2017c), Investing in Climate, Investing in Growth, Éditions OCDE, Paris, https://dx.doi.org/10.1787/9789264273528-en.

OCDE (2017d), Recommandation du Conseil sur les Principes applicables à la gouvernance publique des partenariats public-privé, http://www.oecd.org/fr/gov/budgetisation/PPPRecommandation.pdf.

OCDE (2017e), Multi-level Governance Reforms : Overview of OECD Country Experiences, OECD Multi-level Governance Studies, Éditions OCDE, Paris, https://dx.doi.org/10.1787/9789264272866en.

OCDE (2017f), Key Issue for Digital Transformation in the G20, https://www.oecd.org/g20/key-issuesfor-digital-transformation-in-the-g20.pdf.

OCDE (2016a), Preventing Corruption in Public Procurement, Editions OCDE, Paris, http://www.oecd.org/gov/ethics/Corruption-Public-Procurement-Brochure.pdf.

OCDE (2016b), Examens environnementaux de l'OCDE : France 2016, Examens environnementaux de l'OCDE, Éditions OCDE, Paris, https://dx.doi.org/10.1787/9789264252592-fr.

OCDE (2014a), Examens de l'OCDE des politiques d'innovation : France 2014, Examens de l'OCDE des politiques d'innovation, Éditions OCDE, Paris, https://dx.doi.org/10.1787/9789264214019-fr.

OCDE (2014b), Recommandation du Conseil sur l'investissement public efficace entre niveaux de gouvernement, http://www.oecd.org/fr/regional/politique-regionale/Principes-investissementpublic.pdf.

OCDE (2013), Fiscal Federalism 2014 : Making Decentralisation Work, Éditions OCDE, Paris, https://dx.doi.org/10.1787/9789264204577-en.

OCDE/Banque mondiale/ONU Environnement (2018), Financing Climate Futures : Rethinking Infrastructure, Éditions OCDE, Paris, https://dx.doi.org/10.1787/9789264308114-en.

Parlement européen (2015), Freight on Road: Why EU Shippers Prefer Truck to Train, Rapport, Bruxelles, http://www.europarl.europa.eu/RegData/etudes/STUD/2015/540338/IPOL_STU(2015)540338 EN.p df.

RTE (2017), Bilan prévisionnel de l'équilibre offre-demande d'électricité en France, Rapport, Paris, https://www.rte-france.com/sites/default/files/bp2017_complet_vf.pdf.

Saussier, S. (2017), « Public-private partnerships for infrastructure at the sub-national level of government: Opportunities and challenges in France », EPPP Discussion Paper, vol. 2017-2.

Saussier, S. et J. Tirole (2015), «Renforcer l'efficacité de la commande publique », Note du CAE, vol. 22.

Sénat (2014), Rapport d'information sur les partenariats publics-privés, Rapport, Paris, https://www.senat.fr/rap/r13-733/r13-7331.pdf.

Spinetta, J. (2018), L'avenir du Transport ferroviaire, Rapport, Paris, https://www.ecologiquesolidaire.gouv.fr/sites/default/files/2018.02.15 Rapport-Avenir-du-transport-ferroviaire.pdf. 